\title{
RECEIVED
}

NOV 211995

OAK RIDGE NATIONAL OSTI LABORATORY

\section{FSP (Full Space Parameterization), Version 2.0}

\author{
Gregory A. Fries \\ Charles J. Hacker \\ François G. Pin
}


This report has ben reproduced directly from the best available copy.

Available to DOE and DOE contractors from the Office of Scientific and Technical Information, P.O. Box 62, Oak Ridge, TN 37831; prices available from (615) 576-8401, FTS 626-8401.

Available to the public from the National Technical Information Sorvice, U.S. Department of Commerce, 5285 Port Royal Rd., Springfield, VA 22161.

This report was prepared as an account of work sponsored by an agency of the United States Government. Neither the United States Government nor any agency thereof, nor any of their employees, makes any warranty, express or implled, or assumes any legal liability or responsibility for the accuracy, completeness, or usefulness of any information, apparatus, product, or process disclosed, or represents that its use would not infringe privately owned rights. Reference herein to any specific commercial product, process, or service by trade name, trademark, manufacturer, or otherwise, does not necessarily constttute or imply its endorsement, recommendation, or favoring by the United States Government or any agency thereof. The views and opinions of authors expressed herein do not necessarily state or reflect those of the United States Government or any agency thereof. 
Robotics and Process Systems Division

\title{
FSP (FULL SPACE PARAMETERIZATION), VERSION 2.0
}

Gregory A. Fries, Charles J. Hacker, and François G. Pin

DATE PUBLISHED — October 1995

\author{
Prepared by the \\ OAK RIDGE NATIONAL LABORATORY \\ Oak Ridge, Tennessee 37831 \\ managed by \\ LOCKHEED MARTIN ENERGY SYSTEMS, INC. \\ for the \\ U.S. DEPARTMENT OF ENERGY \\ under contract DE-AC05-84OR21400
}




\section{DISCLAIMER}

Portions of this document may be illegible in electronic image products. Images are produced from the best available original document. 


\section{CONTENTS}

ABSTRACT ....................................................................................................... vii

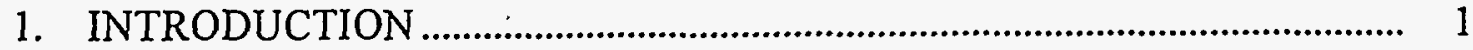

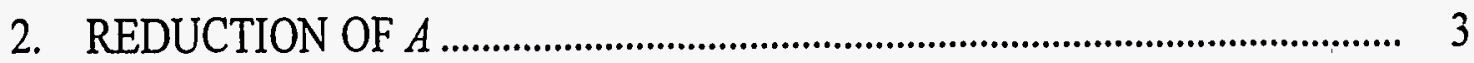

3. GENERAL SOLUTION GENERATION …………………………………......... 7

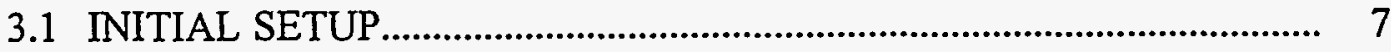

3.2 FINDING THE FIRST SOLUTION................................................................ 9

3.3 ORGANIZING RESULTS........................................................................ 11

4. GENERATION OF REMAINING SOLUTIONS.............................................. 13

5. PROBLEMATIC PARTICULAR SYSTEMS ………………............................. 19

5.1 SPECIALCASE1 ......................................................................................... 19

5.2 SPECIALCASE2 .......................................................................................... 24

6. HANDLING A LOSS OF RANK......................................................................... 29

7. EXAMPLES OF REDUCTION ………………………………………………….... 31

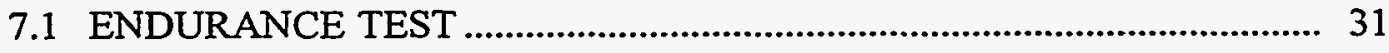

7.2 MULTIPLE RANK LOST ............................................................................... 33

8. RUN TIME TESTING ......................................................................................... 35

9. CONCLUSION................................................................................................. 41

10. ACKNOWLEDGMENT.................................................................................... 43

REFERENCES..................................................................................................... 45

APPENDIX A — USER'S GUIDE ............................................................................. 47

APPENDIX B - CODE LISTINGS........................................................................... 49

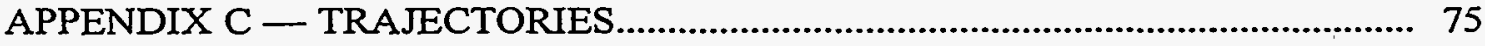

APPENDIX D — TRAJECTORY DATA.................................................................. 81 
$\therefore$ 


\section{LIST OF FIGURES}

Fig.

$\underline{\text { Page }}$

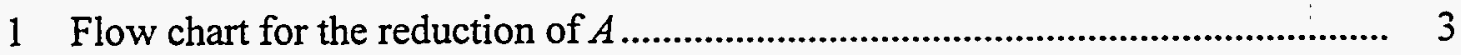

2 Relationships between cases for reduction ......................................................... 4

3 Example of matrix failing reduction in FSPv1 1.0 ................................................ 5

4 Flowchart of outer shell algorithms used to find solution vectors.......................... 8

5 Flowchart of algorithm which finds the first block pattern ................................ 10

6 Tree structure of all possible combinations of blocking solutions $(m=5, n=3) \ldots . .13$

7 Flowchart of the algorithm which recursively finds the remaining solution vectors ........................................................................................................

8 Hypothetical situation in which the values of Tackon are traced........................... 16

9 Hypothetical situation in which the values of FirstOK are traced ........................ 16

10 Timing comparison for the HERMIES manipulator arm ...................................... 35

11 Timing comparison for the AIRARM manipulator arm .................................... 36

12 Timing comparison for the AIRARM manipulator arm...................................... 38

13 Timing comparison for AIRARM manipulator arm ............................................... 38 


\section{LIST OF TABLES}

Table

$\underline{\text { Page }}$

1 Ratio of computation time of FSPv1.0 and FSPv2.0 to pseudo-inverse for the HERMIES configuration..

2 Ratio of computation time of FSPv1.0 and FSPv2.0 to pseudo-inverse for the AIRARM configuration.

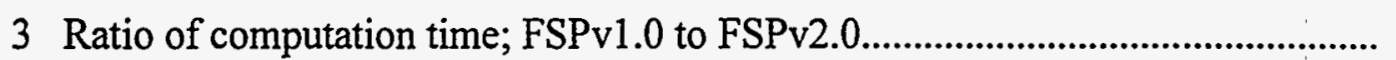

4 Ratio of computation time of FSPv1.0 and FSPv2.0 to pseudo-inverse for the HERMIES configuration with orientation control

5 Ratio of computation time of FSPv1.0 and FSPv2.0 to pseudo-inverse for the AIRARM configuration with orientation control.

6 Ratio of computation time; FSPv1.0 to FSPv2.0, with orientation control........... 39 


\begin{abstract}
This paper describes the modifications made to FSPv1.0 for the Full Space Parameterization (FSP) method, a new analytical method used to resolve underspecified systems of algebraic equations. The optimized code recursively searches for the necessary number of linearly independent vectors that are necessary to form the solution space. While doing this, it ensures that all possible combinations of solutions are checked, if needed, and handles complications which arise due to particular cases. In addition, two particular cases which cause failure of the FSP algorithm were discovered during testing of this new code. These cases are described in the context of how they are recognized and how they are handled by the new code. Finally, testing was performed on the new code using both isolated movements and complex trajectories for various mobile manipulators.
\end{abstract}




\section{INTRODUCTION}

This report is a follow-up report to Enhanced Code for the Full Space Parameterization Approach to Solving Underspecified Systems of Algebraic Equations: Version 1 [1]. The purpose of this approach is to solve Eq. (1) for $x$ when $A$ is a rectangular matrix with more columns than rows:

$$
A \bar{x}=b
$$

Such a system is called underspecified due to the infinite number of solutions for $x$ caused by $A$ having fewer rows than columns.

When used for control of a robotic manipulator system, the $b$ vector represents the $n \times 1$ end-effector displacement vector, the matrix $A$ is the $n \times m$ Jacobian matrix of the system, and $x$ is the $m \times 1$ vector of joint displacements for the $m$ degree of freedom manipulator. When $m>n$, the system is called kinematically redundant. In robotics, a kinematically redundant system is very desirable because it is to one's advantage if a robot has several ways to reach the same point. In particular, such a redundant system has the ability to avoid obstacles and joint limits. To do this, constraints must be placed on the system, limiting several configurations. Typical methods for solving Eq. (1) with these constraints are outlined in Ref. 2. These methods solve the system by either creating a generalized inverse corresponding to specific criteria and then superimposing a homogeneous solution which corresponds to a secondary criteria, or by augmenting the task space with a set of constraints that create an extended Jacobian which can be inverted. However, such methods have difficulties with multiple criteria (task prioritization) and are unable to generate all possible solutions. Thus it would be desirable to have a method which quickly produces all the solutions to Eq. (1) whereupon one can be selected to optimize a certain set of criteria and constraints.

The FSP (Full Space Parameterization) method [3] accomplishes this by finding a parameterized expression for the space of all solutions. The number of linearly independent vectors required to form this space has been determined to be $m-n+1$ [3]. Because the parameterization represents the entire space of solutions to Eq. (1), any particular solution can then be obtained analytically for given conditions (to see a detailed development of the selection of a solution given a set of constraints see Ref. 3).

This paper discusses enhancements made on the version 1.0 of the FSP code. Section 2 describes how reduction of the Jacobian is done in order to eliminate problematic situations and reduce computing time. The next two sections present the method for selection of the $m-n+1$ linearly-independent vectors which span the solution space. Section 5 discusses problematic particular cases that were discovered in the testing of the FSP method. This is followed in Section 6 and 7 by a set of examples illustrating how the code runs for many different situations and demonstrating the ability to handle trajectories which failed with previous versions. Finally, timing tests comparing the FSPv2.0 code with the previous versions are shown in Section 8. 


\section{REDUCTION OF $A$}

The matrix $A$ needs to be reduced for two primary reasons: to eliminate problematic particular cases and to recognize situations in which the matrix can be reduced in order to decrease computational time (e.g., restrictions).

In FSPv2.0, the function ReduceA performs the necessary reduction checks for FSP. The major new features of this version are the insertion of SpecialCasel and SpecialCase 2 and the new iterative nature of the function. The flowchart in Fig. 1 shows the new algorithm of ReduceA.

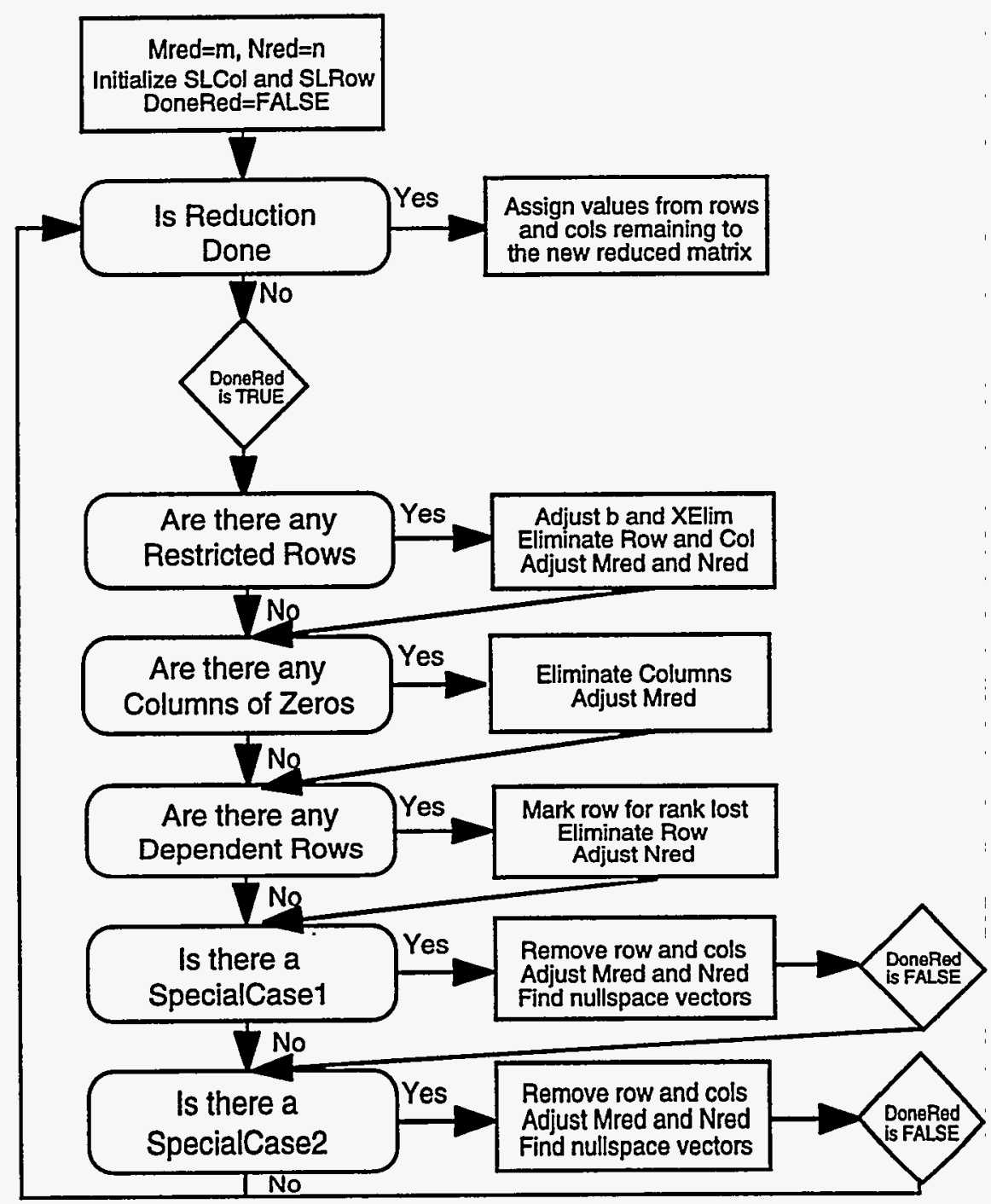

Fig. 1. Flow chart for the reduction of $A$. 
To eliminate the unnecessary timely reassignment of the reduced matrix $A$ every time a need for reduction is found, ReduceA now contains two arrays: SLRow and SLCol. The purpose of these arrays is to keep track of the columns and rows in the original matrix that are not eliminated by reduction. From Fig. 1 it can be seen that this timely assignment to the new reduced matrix is only done once after reduction is complete. To determine when reduction is complete, a new variable DoneRed is used. Each time the outer loop starts, it anticipates completion or reduction by assigning TRUE to DoneRed. Then if a case exists where there may be need for further reduction, DoneRed is changed to indicate this need. Determination of such a need was derived from relationships between the reduction steps as shown in Fig. 2.

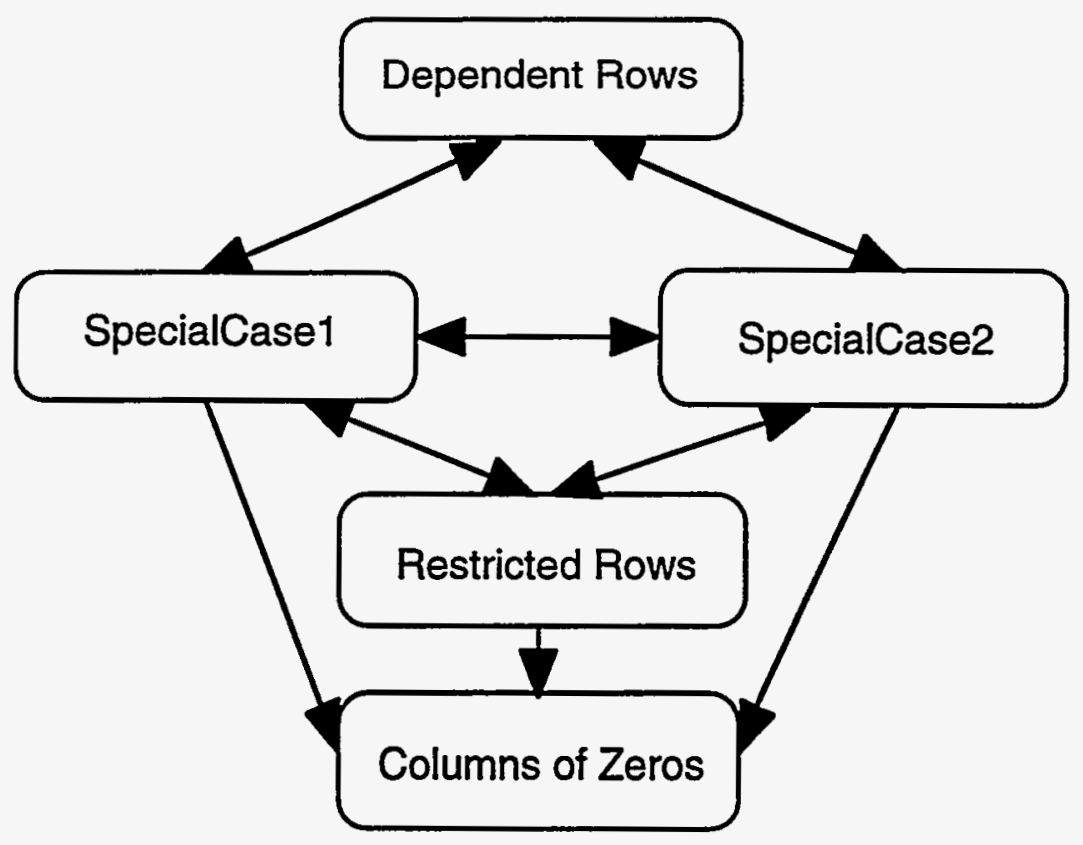

Fig. 2. Relationships between cases for reduction.

In Fig. 2, an arrow from case A to case B indicates that a reduction of case A from a given matrix can potentially cause a case $B$. These five checks are separated into two categories: common occurrences and special cases. Common occurrences consist of Dependent Rows, Restricted Rows, and Columns of Zeros. The remaining two special cases were put into the special case category. The primary reason for such a grouping is because the special cases rarely occur in any real application, thus checking them first would be wasting time if a common occurrence is present, and a special case is not. In addition, the common occurrence tests are much quicker than the special case ones causing much less time wasted if they are repeated. The reason why Columns of Zeros was 
grouped with common occurrences is because completing this before special 'case checks makes the special case checks quicker (see Section 5).

Following Fig. 1, one can see that once inside the reduction loop the check for restricted rows is performed. This check uses the same algorithms used in the ReduceA procedure of FSTV1.0 [1]. The major change, though, is the references to SLCol, and SLRow positions in the original matrix, rather than positions in a temporary copy of the original. Next, any columns of zeros are eliminated from consideration. Then row dependency is checked. This check is performed by calculating the nullspace of the transpose of the current matrix, $A^{T}$. The method used to perform this operation is Singular Value Decomposition (SVD) as described in Ref. 1. One major problem in FSPv1.0 was the recognizing of such a case when it did not occur. An example of such a matrix which caused the invalid reduction is shown in Fig. 3.

$$
\left[\begin{array}{llllllllll}
1 & 0 & 0 & 0 & 0 & 0 & 0 & 1 & 0 & 0 \\
0 & 0 & 0 & 0 & 0 & 0 & 0 & 0 & 1 & 1 \\
0 & 1 & 0 & 0 & 1 & 0 & 0 & 0 & 0 & 0 \\
0 & 1 & 1 & 0 & 0 & 0 & 0 & 0 & 0 & 0 \\
0 & 0 & 0 & 1 & 0 & 0 & 1 & 0 & 0 & 0 \\
0 & 0 & 1 & 0 & 1 & 1 & 0 & 0 & 0 & 0
\end{array}\right]
$$

Fig. 3. Example of matrix failing reduction in FSPv1.0.

For this matrix, FSPv1.0 would deduce that row 2 was a dependent row and subsequently would eliminate it causing a serious problem by eliminating the last two degrees of freedom. The reason for such a failure was due to the interface of the transposed matrix and the functioning of the SVD library function. The procedure which uses SVD is called mat_null. This procedure first accesses the type of matrix it is given. The types are organized into square, less rows than columns, and more rows than columns. Due to its nature, the final case cannot be handled by mat-null. To prevent crashing, mat_null handles it by truncating the matrix, making it a square matrix. In relation to ReduceA, it is now obvious why problems would occur due to the transpose of $A$ always having more rows than columns. The reason why row 2 was determined to be dependent in Fig. 3 was because mat_null saw row 2 (changed to column 2 when transposed) as all zeros.

To solve the interface problem between ReduceA and mat_null, FSPv2.0 converts the rectangular transposed matrix into a square one by adding on columns of zero. The value zero was chosen because we are assured a nullity equal to the number of added columns if the matrix $A$ has no dependent rows. Thus for the matrix in Fig. 3, four columns of zeros would be added onto the transposed matrix, and thus mat_null 
would not truncate it. The resulting outcome would be mat_null returning a nullspace of dimension 4 which would be ignored due to the addition of the columns of zeros. If any rows were dependent, mat_null would return a nullspace with greater dimension, and the appropriate row would be eliminated.

The final two special case checks are done next. The recognition and description of these two cases are discussed in Section 5. The main feature of these two cases in the context of ReduceA is their relation to the stopping variable DoneRed. If ever one of these cases is detected, the variable DoneRed is changed so that possibilities for further reduction are examined. The reason for such a step is that the occurrence of either special case and the subsequent reduction of the matrix may cause any of the other reduction cases to occur on the reduced matrix.

After completing the main loop without detecting a special case, ReduceA finally creates the reduced matrix. This matrix is formed from the values in the original matrix whose positions are stored in the arrays SLRow and SLCol. For examples of complete runs and endurance testing of this reduction algorithm see Section 7. 


\section{GENERAL SOLUTION GENERATION}

FSPv2.0 contains two procedures which generate the needed $m-n+1$ linearly independent solution vectors. The first procedure is called Solution_generator. It is the main procedure which drives FSP and can be called by other program segments. The second procedure, which Solution_generator calls, is called RestofSoln. This procedure creates the remaining solution vectors after the first one is created (see Section 4).

The main FSP driver, Solution_generator, can be thought of as an outer shell which monitors and controls all aspects of creating the solution vectors needed. Figure 4 shows a flowchart of the general algorithm of Solution_generator which can be split into three segments: initial setup, finding first solution, and organizing results.

\subsection{INITIAL SETUP}

The first step in setting up Solution_generator is the declaration and allocation of memory for key variables. The following paragraphs will introduce each of these variables and discuss their purpose.

Tackon is the first array created. This array keeps track of what columns are not in the current blocking pattern. In this outer shell these numbers are in lexigraphical order, but its order changes in the RestofSoln procedure when columns from it are substituted in. This variable is key to insuring linear independence of all solution vectors (see Section 4).

Complementing Tackon is the variable, block. This variable contains which columns are to be used for a square matrix to generate a solution. Thus block must be a twodimensional array containing (Mred-Nred +1 ) vectors of length (Nred). Notice how it uses the reduced values of $m$ and $n$ : allocation is needed only after reduction and if blocking is needed.

Yet another variable, FirstOK, is an array that corresponds to the values in the first solution. The values in it are either TRUE or FALSE denoting if the value is non-zero or zero. This array is also essential for the insurance of all solution vectors being linearly independent. In Section 4 it will be seen how this array changes when more solutions are generated.

The two variables ColElim and RowElim are essential elements in keeping track of what has been done to the original matrix during reduction. Each position in the arrays correspond to a row or column in the original matrix. If a row was removed due to being all zeros, or due to it containing a restriction, it will be assigned the value "1" in RowElim. The same is true for a column which is zero or is part of a restriction. If a row is dependent on another row, the row will be eliminated and now RowElim will contain the value " 2 ". When a special case is detected the row eliminated will be assigned the value " 1 ", and the columns will be assigned " 2 " if it is a SpecialCase1, or "3" if it is a SpecialCase2. All rows and columns not eliminated by reduction will contain the original value " 0 ". 


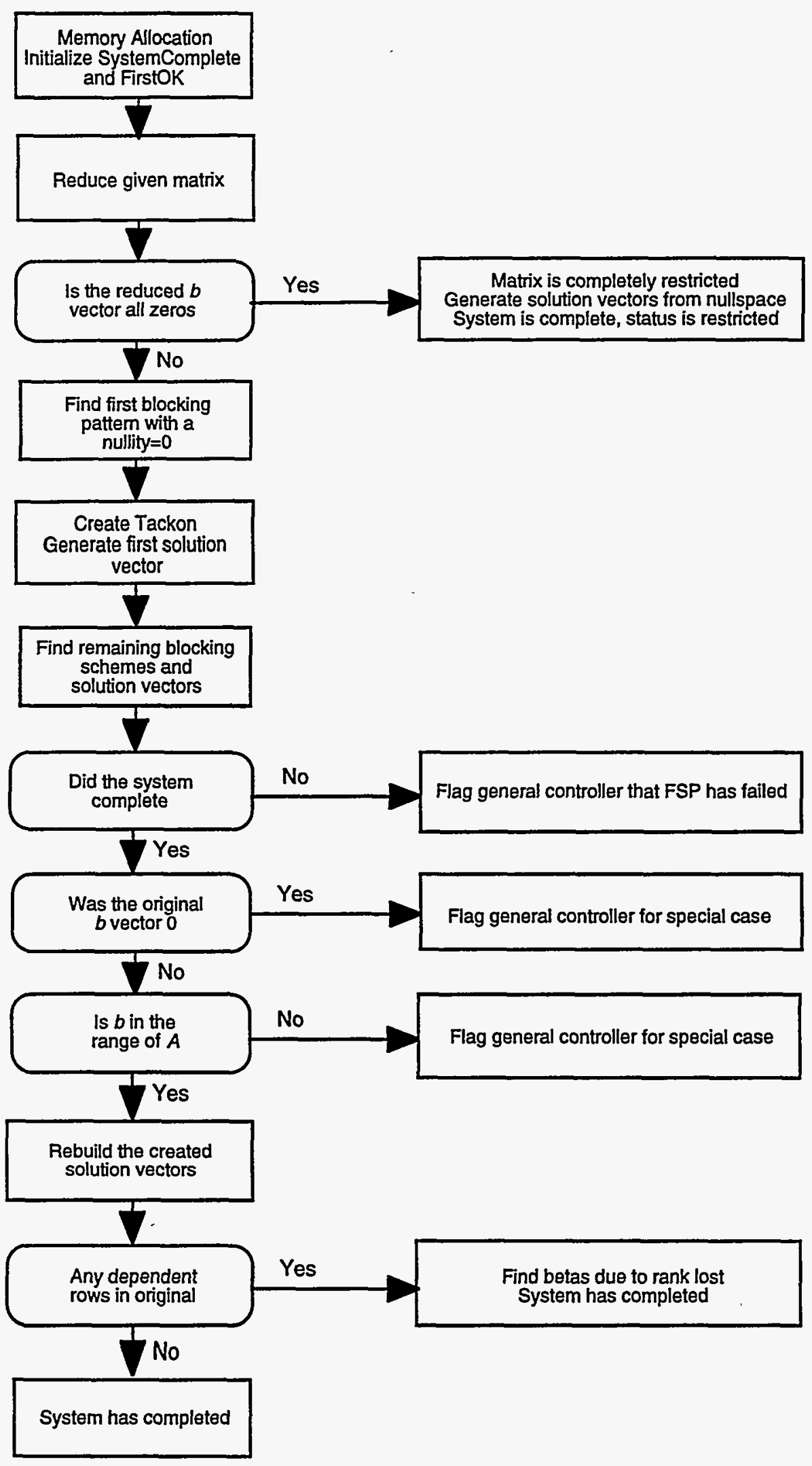

Fig. 4. Flowchart of outer shell algorithm used to find solution vectors. 
One of the major purposes of ColElim and RowElim is the formation of the matrices Ared and bred which are the reduced $A$ and $b$ matrices. They are formed from the elements in $A$ and $b$ corresponding to zero values in ColElim and RowElim. These reduced matrices are the ones used in the formation of the solution vectors.

For reducing cases which decrease the number of vectors, the arrays Xelim and Specialg keep track so that a proper solution is generated. Xelim is used to store a value in the $x$ vector when it is restricted and thus is a vector of length $m$. Specialg contains the extra solution vectors generated when a special case is reduced out. Thus Specialg is a two-dimensional array with dimension: (number of special case solution vectors) $\times m$.

After allocating memory for these data storage elements, some of them are initialized, including SystemComplete, which denotes if the system has found a set of legitimate solution vectors. The next step is calling the reduction procedure which returns a well-conditioned, reduced Jacobian.

Before searching for solution vectors there is one last check. This check is to see if the $b$ vector is comprised of all zeros. If this occurs the system would be fully restricted and $(m-n)$ solution vectors can be obtained from the nullspace of the reduced Jacobian. Thus as long as the original system was not restricted (see check in Section 3.3), the Solution_generator is complete with a valid set of vectors.

\subsection{FINDING THE FIRST SOLUTION}

In Ref. 3, Pin et al. describe an algorithm in which the $(m-n+1)$ linearly independent solution vectors can be found. The first step in this algorithm is the finding of the first well-conditioned square submatrix of $A$. This submatrix consists of $n$ linearly independent columns which are determined to be satisfactory with the function mat_null. The iterative algorithm in which the program searches for this submatrix in lexigraphical order is shown in Fig. 5.

This search starts by creating the first submatrix with the first $n$ columns: $\left[c_{1}, c_{2}, \ldots, c_{n}\right]$. With this submatrix, mat_null is called to find the nullity of the matrix. If the nullity of this matrix is zero, a first blocking pattern is found, and a first solution can be created. If not, it must search for another square submatrix which has a nullity of zero.

To search through the remaining possible square submatrices, an algorithm described in Ref. 4 is used. This algorithm, as seen in Fig. 5, adds 1 to the last blocking position not at its maximum value. Then each position after that one equals the previous position's value plus 1. Such an algorithm would create a sequence of blocks for an $m=5$ and $n=3$ as follows: $1,2,3 ; 1,2,4 ; 1,2,5 ; 1,3,4 ; 1,3,5 ; 1,4,5 ; 2,3,4 ; \ldots ; 3,4,5$. After the next blocking

pattern is created, a submatrix is formed in accordance with the pattern and checked for its nullity. If the nullity is not zero, it continues the search until one such submatrix is found. 


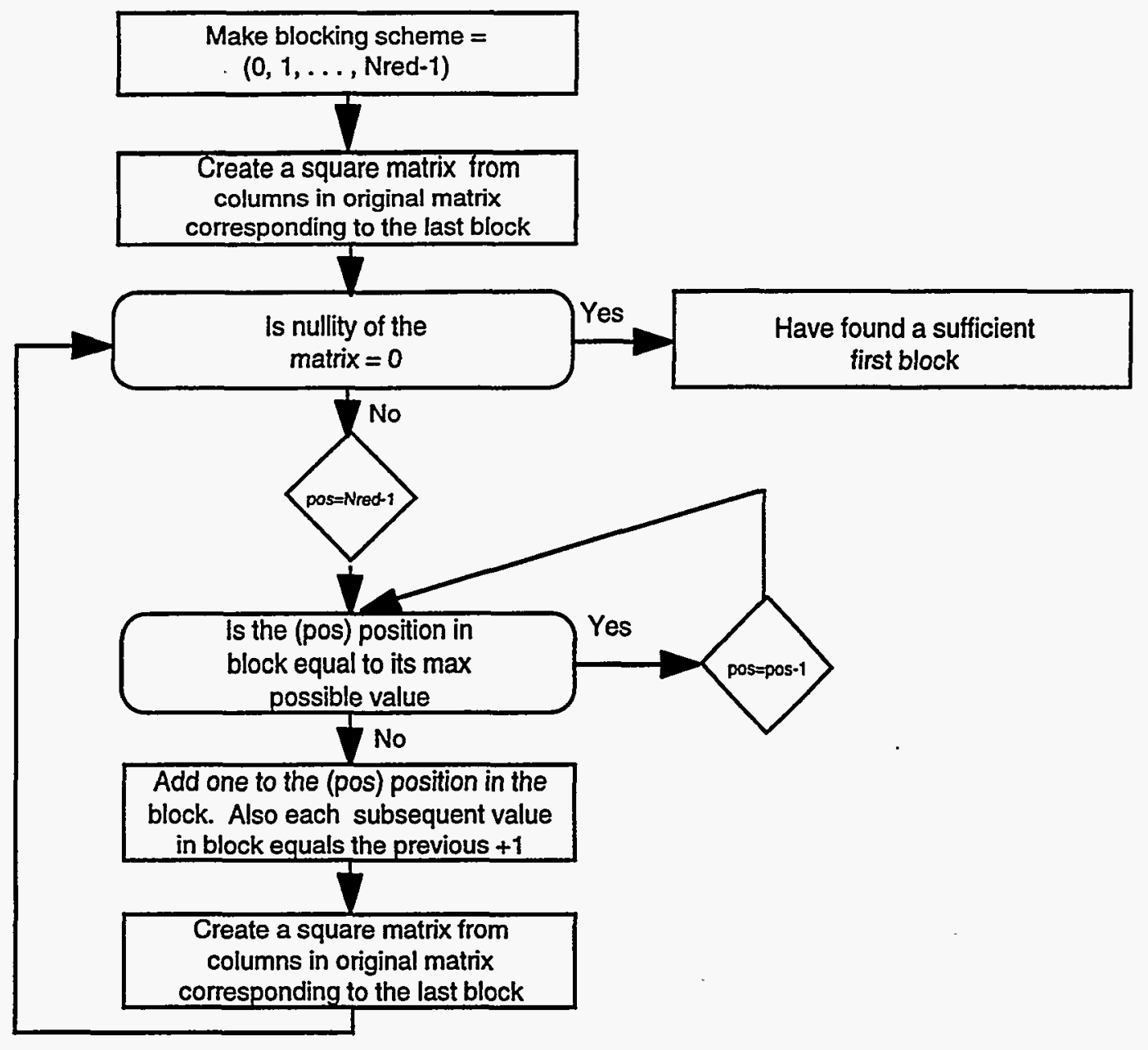

Fig. 5. Flowchart of algorithm which finds the first block pattern.

This part of FSPv2.0 contrasts with FSPv1.0 in two major ways. First the blocking in FSPv1.0 denotes columns which are not used rather than columns used, as in FSPv2.0. The second major difference is that FSPv2.0 uses a value $\mathrm{K} 2$ (the ratio of largest to smallest singular values) to be less than a set value equal to 10 in addition to checking that the nullity $=0$ for a first blocking pattern. The major problem with such a test was that sometimes a submatrix with nullity $=0$ and $\mathrm{K} 2<10$ was never found, and as a default the search would choose the last possible blocking pattern. Aside from the obvious time in searching through all the possible combinations of a first blocking pattern, a major error can occur if that last default block selected has a nullity not equal to zero. The new searching method in FSPv2.0 (see Section 4) allows for the dismissal of this K2 check, and thus reduces the total run time. 


\subsection{ORGANIZING RESULTS}

The remainder of the outer shell is also split into two sections: preparation for creating solution vectors and returning status of results. The preparation for creating the remaining solutions mainly consists of creating the first Tackon array and creating the first solution vector from this blocking pattern. It then calls the procedure RestofSoln (discussed in Section 4) which creates the remaining (Mred-Nred) solution vectors.

The final and most important section of this code tells the driving program segment the status of the completion of FSPv2.0. The first check is to see if the system has completed. If it has, it proceeds to check if the original $b$ vector was not comprised of all zeros, and that it was in the range of $A$. If these both check out, the procedure continues by rebuilding the solution vectors. It does this by substituting zeros in for columns that have been eliminated and adding on the special case solutions generated. Next it checks if RowElim contains any values equal to "2", denoting a loss of rank. If this occurs, Beta values (restrictions) are created as discussed in Section 6. 


\section{GENERATION OF REMAINING SOLUTIONS}

According to Pin et al. [3] once a first solution vector is found, the remaining $(m-n)$ linearly independent solution vectors may be created by substituting the unused columns from the first block in for one column in that block. For instance consider a first invertible square submatrix $J_{1}=\left(c_{1}, \ldots, c_{n}\right)$. Since each of the remaining columns, $c_{i}, i \in\{n+1, \ldots, m\}$ of $A$ is a linear combination of the first $n$ columns, the column $c_{n+1}$ can be represented as $c_{n+1}=\left(\alpha_{1} c_{1}+\ldots+\alpha_{n} c_{n}\right)$. Thus as long as column $c_{l}$ is in the decomposition of column $c_{n+1}$ with $\alpha_{l} \neq 0$, a new invertible submatrix $J_{2}$, with $\operatorname{det}\left(J_{2}\right) \neq 0$, can be formed by swapping column $c_{l}$ of $J_{1}$ with column $c_{n+1}$ of $A$.

Now $J_{2}=\left(c_{1}, \ldots, c_{l-1}, c_{n+1}, c_{l+1}, \ldots, c_{n}\right)$ may be inverted to form a solution to the system. As long as the component corresponding to the column $c_{n+1}$ in the solution vector does not equal zero and the column which $c_{n+1}$ replaces has a non-zero value in the previous solution vector, this new vector $g_{2}$ is guaranteed to be linearly independent from the first solution $g_{1}$. This process can be repeated with the remaining $m-(n+1)$ columns not used, so that $m-n+1$ linearly independent solution vectors are created.

Thus, by this algorithm the number of possible solutions can be represented in a tree-like structure. An example of such a structure is shown in Fig. 6 in which $m=5$, $n=3$, with a first block equal to: $1,2,3$.

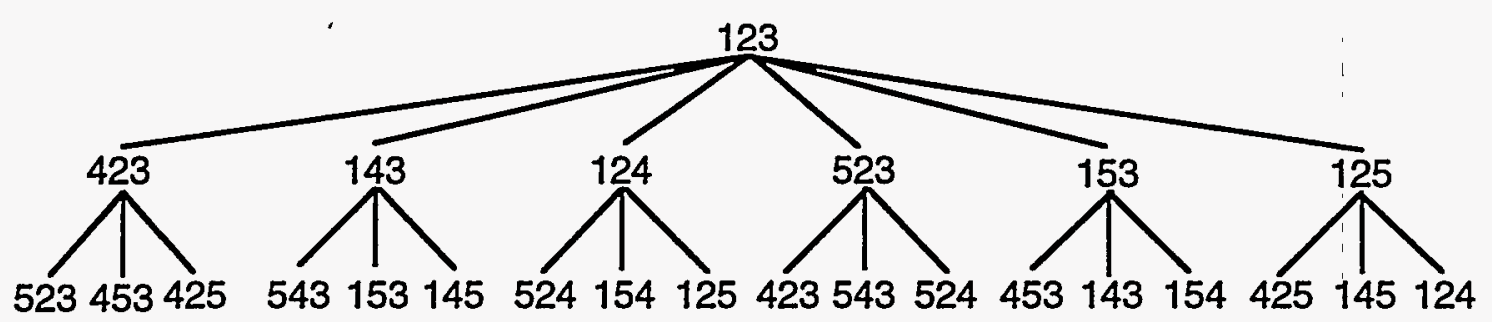

Fig. 6. Tree structure of all possible combinations of blocking solutions $(\mathrm{m}=5, \mathrm{n}=3)$.

Any path through the tree in Fig. 6 from one end to the other represents a possible set of three blocking patterns needed to find the three solution vectors. In addition, any program that is to search all possible branches must be able to handle any combination of $m$ and $n$. FSPv1.0 handled the problem by changing the first blocking pattern after a certain number of subbranches had been checked. FSPv2.0 uses an entirely different recursive algorithm which directly follows the tree in Fig. 6.

What is meant by recursive is that the same procedure is called by itself several times. Once the procedure completes without calling itself, it returns to the previous call. Thus in the tree structure in Fig. 6, the program will be first called with the root 123. 
Next it will be called again with the root 423 , then will check the blocks 523,453 , and 425. If none of these will produce a linearly independent vector, it will return to the level with root 123 and call itself again with the root 143. This recursive nature will continue until it finds a third blocking pattern or has been depleted of all patterns.

The internal workings of this recursive procedure can be seen in Fig. 7 which depicts a flowchart of it. It should be noted that when it calls itself, yet another page is stacked on top of the flowchart. Once a flowchart completes, it returns to the place in the last flowchart it was called from. The advantage of such a system is that each page in the stack contains a memory of where it has been and the corresponding values in the variables at that time.

Every time the function is called, it allocates memory for the arrays Newtack and Changed. The purpose of these arrays is to ensure that the values in Tackon and FirstOK remain constant in each level of recursion. Thus rather than passing them to the next level where they could be changed, copies of them are used. Such a problem occurs only with matrices and arrays for which a memory address is passed rather than a value as in the passing of variables. In addition to allocating memory, the function increments the variable NextToFind. This signifies that the next solution is sought, and the current value of NextToFind is now equal to the number of solutions that have been found (number of recursive level currently in).

The next variable assigned is $\mathrm{X}$ which denotes the position in Tackon which is going to be examined for substitution in for one of the columns in the last blocking pattern. The reason why X is set equal to NextToFind-1 is because the first index in the array is "0". In addition once a value in a block has been substituted in for, it is placed in the first position of Tackon. For example, with the tree in Fig. 6, suppose that the first branch system is a solution: $1,2,3 ; 4,2,3 ; 5,2,3$. In such a scenario, the values of the array Tackon for each level are shown in Fig. 8. Level "1" denotes the first calling of the procedure where it should look at index " 0 " due to the fourth row not being used in any blocking pattern. For recursion level 2, the value in index " 0 " is now 1 denoting that column 1 has been subbed in. Therefore index " 1 " containing the value 5 must now be looked at.

The next major step of the procedure is the modification of the array FirstOK. The main purpose of this array is to ensure that solution vectors are linearly independent. The conditions which must be fulfilled for each new solution to be linearly independent of the previous solutions are given in Refs. 1 and 3. The algorithm used here always substitutes a new column into the place of a column of the first solution.

To ensure linear independence of all solution vectors, a column corresponding to a zero component of a solution vector should not be substituted. The entries in FirstOK mark whether or not each zero element in the first solution (except those from columns not in the inverted block) corresponds to a non-zero element in some other solution. For example given the situation of Fig. 8, suppose the first solution vector contains $\left[\begin{array}{llll}\mathrm{X} & 0 \mathrm{X} & 0 & 0\end{array}\right]$, the second $[0 \mathrm{X} 0 \mathrm{X} 0]$, and third $[0 \mathrm{XX} 0 \mathrm{X}]$, where $\mathrm{X}$ denotes a non-zero value. Figure 9 shows how the array FirstOK reacts to such a scenario. 
Allocate memory

Increment NextToFind

Assign $X=$ NextToFind - 1

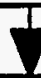

Reset FirstOK with

Current solution vector

Copy FirstOK into Changed

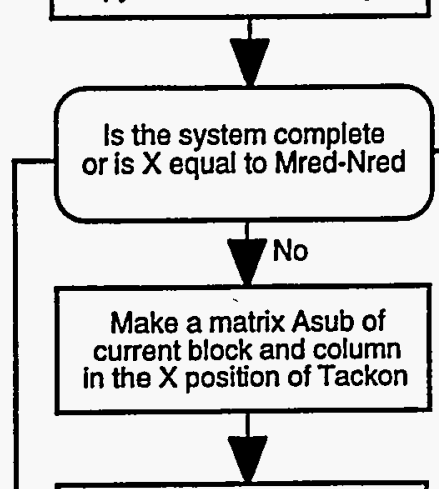

Make a matrix Asub of

current block and column

in the $X$ position of Tackon

Find nullspace vector of Asub

Set $Y$ equal to zero

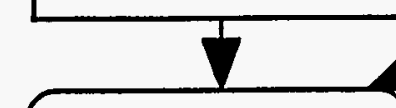

Is the system complete or is $Y$ equal to Nred
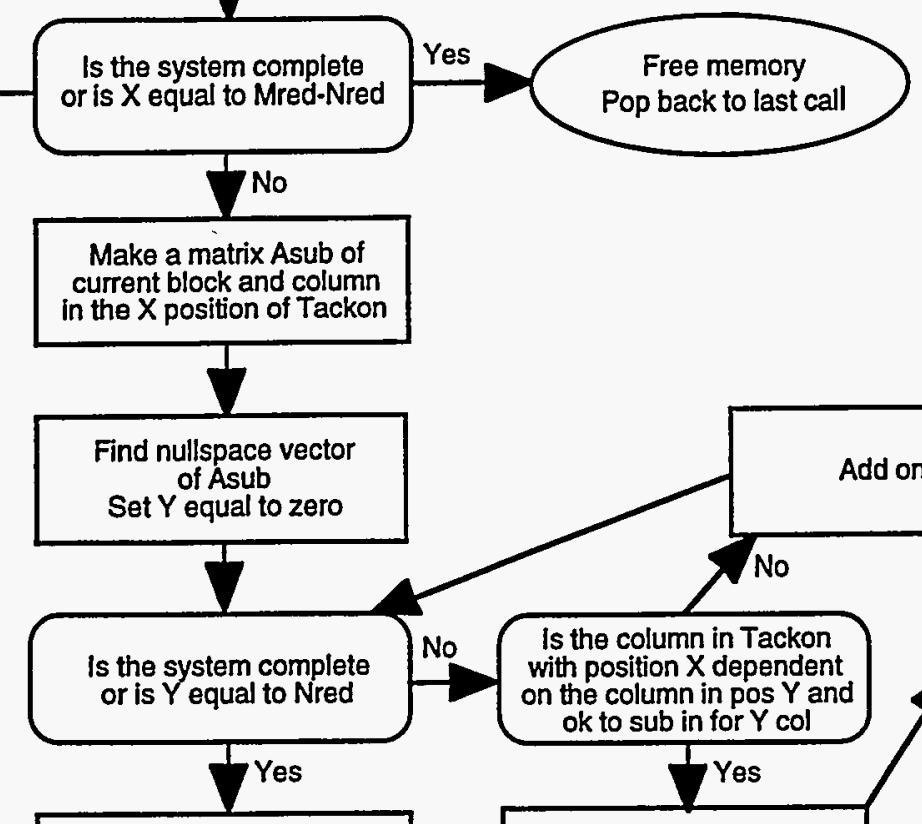

Add one to $X$

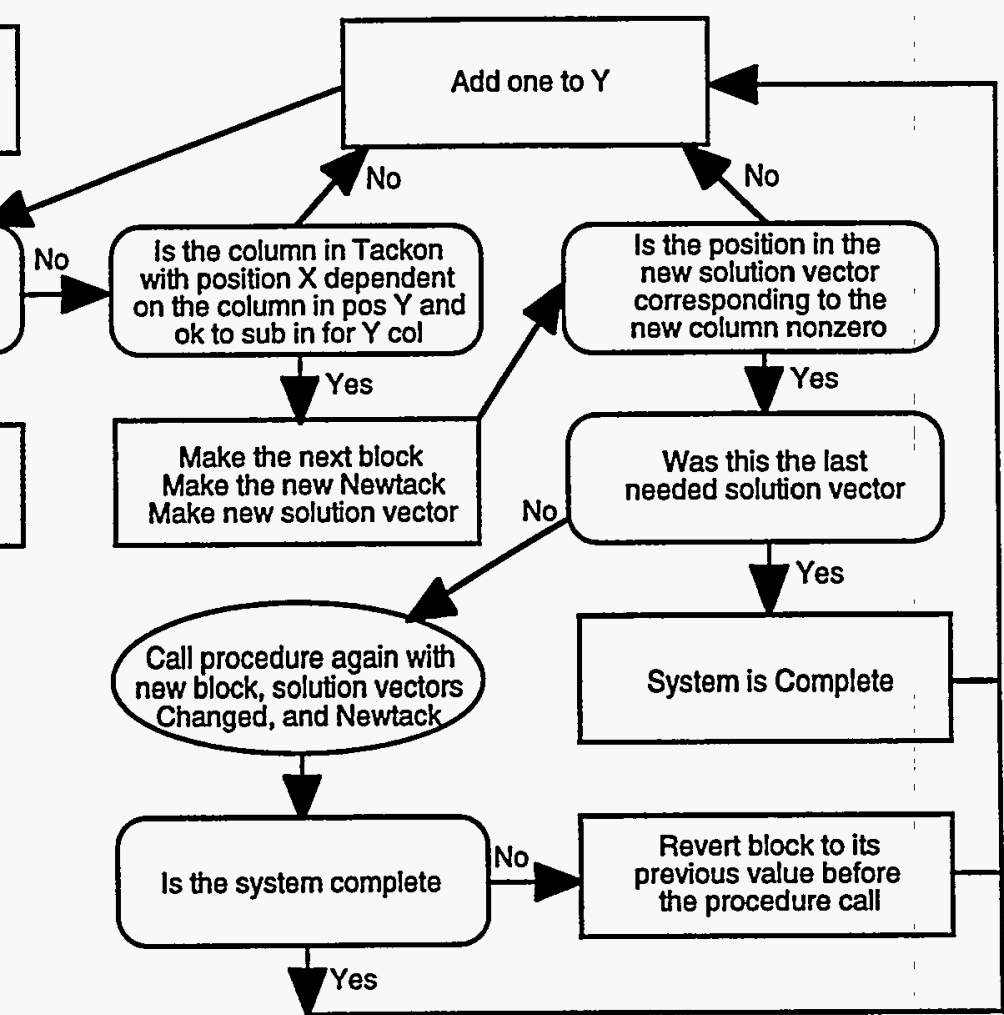

Fig. 7. Flowchart of the algorithm which recursively finds the remaining solution vectors. 
Level 1

\begin{tabular}{|l|l|l|}
\hline index & 0 & 1 \\
\hline Tackon & 4 & 5 \\
\hline
\end{tabular}

Level 2

\begin{tabular}{|l|l|l|}
\hline index & 0 & 1 \\
\hline Tackon & 1 & 5 \\
\hline
\end{tabular}

Level 3

\begin{tabular}{|l|l|l|}
\hline index & 0 & 1 \\
\hline Tackon & 1 & 4 \\
\hline
\end{tabular}

Fig. 8. Hypothetical situation in which the values of Tackon are traced.

Level 1

\begin{tabular}{|l|l|l|l|}
\hline index & 0 & 1 & 2 \\
\hline FirstOK & 1 & 0 & 1 \\
\hline
\end{tabular}

Level 2

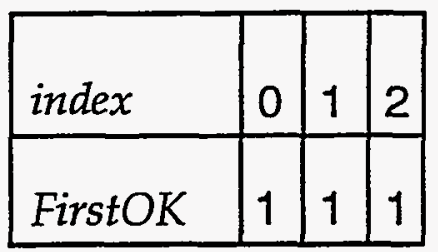

Level 3

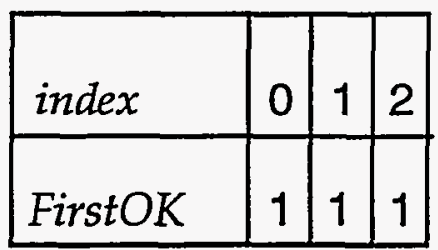

Fig. 9. Hypothetical situation in which the values of FirstOK are traced.

For this situation the first block creates a solution with a zero value in column two. Thus in level 1 FirstOK has a zero value in the position corresponding to the second column. Once the second solution is generated the second column now has a non-zero value so that now all the columns in the first blocking scheme are able to be substituted in. This remains true in the third level. It should also be noted that FirstOK only applies to the first blocking scheme because when a column is substituted in, it must contain a nonzero value in its place in the new solution vector. The modification of FirstOK works in the recursive algorithm by checking all the indices to see what columns cannot be substituted. If a column passes such a test, that column's position is checked in the last solution vector generated to see if its status has been changed.

After FirstOK has been set, the first, or outer, loop is entered, which increments $\mathrm{X}$ until it equals Mred-Nred. In effect, this loop compares every column not used in a blocking pattern with the current block (values in Tackon). Once it has exhausted all these possibilities the branch it is in must be exited, and it returns to the previous level.

To compare each of these columns with the current block, an $n \times(n+1)$ matrix, Asub, is formed. Asub is comprised of the current blocking columns plus the column that is going to be compared. Next the nullspace vector of Asub is found with the mat null function. If a column in the current block is part of the linear decomposition of the column in question, the position in the nullspace vector of that column will be non-zero. To see which columns in the block are in the decomposition yet another, inner, loop is entered. The inner loop counter is $Y$ which begins at the value 0 and stops once it reaches the last position in the block, Nred.

The algorithm next performs three checks to see if a substitution is valid. These checks see if the columns in question are dependent (nullspace vector check), if the 
column to be substituted satisfies the FirstOK condition, and if the next solution vector created by the substitution will have a non-zero value in the position substituted. If these tests are passed, then the system checks if this solution vector is the last one needed. If it is, then the system is complete, and the global variable SystemComplete becomes TRUE, otherwise the procedure is called again in order to find the remaining solutions. The variable SystemComplete is made to be global, so that when it does become true all levels of recursion see that FSP is done, and it no longer needs to find more solutions. 


\section{PROBLEMATIC PARTICULAR SYSTEMS}

This section describes two particular cases which caused the previous FSP algorithm to fail. In each section a detailed description of the case is followed by an explanation of how the matrix is reduced to eliminate the problem. In both cases failure is due to an inability of the algorithm to substitute all remaining columns into the square submatrix (i.e. inability to include a column in subsequent blocking patterns). This results in a deficiency in the number of linearly independent solution vectors.

The following two subsections present solutions to the two major algorithmic problems which have been found. Note that a numerical problem may still occur. If columns of $A$ contain all significantly large or all significantly small elements, a complete set of solution vectors will not be found since these columns will create non-invertible square submatrices. A solution for this problem is currently under development.

\subsection{SPECIALCASE1}

During testing of the algorithm, a particular case, called SpecialCase1, caused difficulty finding the needed $m-n+1$ linearly independent solution vectors. This particular case can only occur when the value in the $b$ vector, corresponding to a row $i$, contains the value zero. If all non-zero values in the row $i$ belong to columns that are multiples of each other, i.e., are linearly dependent, the algorithm will fail. The reason for this failure is due to the fact that all matrices containing two or more of these columns are not invertible, which requires that all the invertible matrices contain only one of these columns. Since the corresponding value in $b$ is zero, we are assured that all solution vectors created will have zeros in all the columns which corresponded to the non-zero values in the original matrix. Thus only $m-n+1$-(the number of non-zero columns) linearly independent vectors can be created.

For example given the equation $A x=b$ as follows:

$$
\left|\begin{array}{ccccc}
A_{11} & A_{12} & A_{13} & \cdots & A_{1 m} \\
A_{21} & A_{22} & A_{23} & \cdots & A_{2 m} \\
A_{31} & A_{32} & A_{33} & \cdots & A_{3 m} \\
\vdots & \vdots & \vdots & \ddots & \vdots \\
A_{n 1} & A_{n 2} & A_{n 3} & \cdots & A_{n m}
\end{array}\right|\left|\begin{array}{c}
X_{1} \\
X_{2} \\
X_{3} \\
\vdots \\
X_{m}
\end{array}\right|=\left|\begin{array}{c}
b_{1} \\
b_{2} \\
b_{3} \\
\vdots \\
b_{n}
\end{array}\right|
$$

Suppose that a given element in $b$ is equal to zero: $b_{i}=0,1 \leq i \leq n$. Thus Eq. (2) is produced:

$$
A_{12} X_{1}+A_{i 2} X_{2}+A_{i 3} X_{3}+\cdots+A_{i m} X_{m}=0
$$


Now suppose that a given number, $j_{a}$, of columns in the above matrix, $A$, are multiples of each other

$$
j_{a}, j_{a} \in\{1 \cdots m\}
$$

where $a=\{1, \ldots, k\}$ and $k=$ number of dependent columns in $A$.

Due to the characteristic of these columns being multiples of each other, all dependent columns $j_{a}$ can be related to the column $j_{1}$ by a constant value $C_{a}$, producing the following relations:

$$
j_{1}=C_{1} j_{1}, j_{2}=C_{2} j_{1}, \ldots, j_{k}=C_{k} j_{1}
$$

Now all columns not contained in the set $j_{a}$ can be included in a similar set of nonmultiple columns, $r$, with elements $r_{b}$, where $1 \leq b \leq s$, and $s=$ number of non-multiple columns in $A$. Thus Eq (2) may be modified into the following form:

$$
A_{i j_{1}} X_{j_{1}}+A_{i j_{2}} X_{j_{2}}+\cdots+A_{i j_{k}} X_{j_{k}}+A_{i r_{1}} X_{r_{1}}+A_{i r_{2}} X_{r_{2}}+\cdots+A_{i r_{s}} X_{r_{s}}=0
$$

If all values corresponding to the columns $r_{b}$ are zero in row $i$ of matrix $A$, Eq. (4) may be reduced to

$$
A_{i j_{1}} X_{j_{1}}+A_{i j_{2}} X_{j_{2}}+\cdots+A_{i j_{k}} X_{j_{k}}=0
$$

Equation (5) can be further reduced using Eq. (3) and the fact that $C_{1}=1$.

$$
\begin{gathered}
A_{i j_{1}} X_{j_{1}}+C_{2} A_{i_{1}} X_{j_{2}}+C_{3} A_{i_{1}} X_{j_{3}}+\cdots+C_{k} A_{i j_{1}} X_{j_{k}}=0 \\
X_{j_{1}}+C_{2} X_{j_{2}}+C_{3} X_{j_{3}}+\cdots+C_{k} X_{j_{k}}=0
\end{gathered}
$$

The remaining rows not categorized by SpecialCasel can be denoted as $p$. It can be seen that all such rows can be broken down into the same form as Eq. (4), except that now the sum may equal a non-zero value $b_{p}$. By a similar process used to reach Eq. (6), the remaining products are reduced to the form

$$
A_{p j_{1}}\left(X_{j_{1}}+C_{2} X_{j_{2}}+C_{3} X_{j_{3}}+\cdots+C_{k} X_{j_{k}}\right)+A_{p r_{1}} X_{r_{1}}+A_{p r_{2}} X_{r_{2}}+\cdots+A_{p r_{s}} X_{r_{s}}=b_{p}
$$

This can be reduced even further using Eq. (6):

$$
A_{p r_{1}} X_{r_{1}}+A_{p r_{2}} X_{r_{2}}+\cdots+A_{p r_{s}} X_{r_{s}}=b_{p}
$$


Equation (8) shows that for the given SpecialCase1, the values of multiple columns do not affect the equations created by the products of non-SpecialCase1 rows. With this knowledge, a scheme was developed which reduced the matrix so the SpecialCasel was no longer prevalent. Since the columns do not factor into any other equations, they were eliminated, creating a row of zeros which was also eliminated. For instance if a SpecialCasel occurred for one row and four columns in an $n \times m$ matrix the new matrix would be $(n-1) \times(m-4)$ resulting in $m-n-2$ independent solution vectors. The remaining three vectors needed to span the space are acquired from the nullspace of the non-zero multiple column values in the removed row.

An example of such a test run occurs with the following matrix $A$ and vector $b$, in which one row and two columns are reduced out:

$$
\begin{gathered}
A=\left[\begin{array}{rrrrrrrrr}
0.0000 & 0.0000 & 0.0000 & 0.0000 & 0.0000 & 0.0000 & 1.0000 & 0.0000 & -0.6100 \\
-1.2360 & 0.6350 & 0.8800 & 0.0000 & 0.0000 & 0.3430 & 0.0000 & 1.0000 & -0.8930 \\
0.0000 & 0.0000 & 0.0000 & -0.8510 & -0.3430 & 0.0000 & 0.0000 & 0.0000 & 0.0000
\end{array}\right] \\
b=\left[\begin{array}{lllll}
-0.0101 & 0.0000 & 0.0000
\end{array}\right]^{T}
\end{gathered}
$$

With the reducing scheme $A$ becomes:

$$
\left[\begin{array}{rrrrrrr}
0.0000 & 0.0000 & 0.0000 & 0.0000 & 1.0000 & 0.0000 & -0.6100 \\
-1.2360 & 0.6350 & 0.8800 & 0.3430 & 0.0000 & 1.0000 & -0.8930
\end{array}\right]
$$

Thus row 3 and columns 4 and 5 have been eliminated due to columns 4 and 5 being multiples of each other and the only non-zero components of row three. The algorithm checks for such a case by first examining the $b$ vector. If a value is zero, it must check all columns by testing all possible combinations, using only 2 columns at a time, for multiplicity (for condition of multiplicity see Eq.(2)). Once a pair of these columns are found not to be multiples of each other, the algorithm determines that a SpecialCasel condition does not occur for that row, and moves to next element in the $b$ vector.

For instance with the test matrix described above, row 1 is skipped due to the first position in the $b$ vector being non-zero. Next, row 2 alerts the SpecialCasel check due to a zero value in the $b$ vector. The check starts by comparing columns 1 and 2 which satisfy the condition for multiplicity. Next columns 1 and 3 are checked, then columns 1 and 6 , and then columns 1 and 8 are checked. Finally, when columns 1 and 9 are checked, the columns are determined not to be multiples of each other, and thus SpecialCase1 does not occur for row 2. If column 9 were $\left[\begin{array}{lll}0 & 1 & 0\end{array}\right]^{T}$., then here would have been a SpecialCase 1 for row 2 , and columns $1,2,3,6,8$, and 9 would be eliminated in addition to row 2. 
When row 3 is checked, there is a SpecialCasel. The reason for this is that all the non-zero values correspond to columns which are multiples of each other (columns 4 and 5). Now that this condition is recognized, the necessary solution vectors for the reduced matrix are produced. The program easily handles the reduced case, and for the given test case it creates the solution vectors.

$$
\left[\begin{array}{rrrrrrr}
0.0000 & 0.0000 & 0.0000 & 0.0000 & -0.0101 & 0.0000 & 0.0000 \\
-0.0120 & 0.0000 & 0.0000 & 0.0000 & 0.0000 & 0.0000 & 0.0166 \\
0.0000 & 0.0233 & 0.0000 & 0.0000 & 0.0000 & 0.0000 & 0.0166 \\
0.0000 & 0.0000 & 0.0168 & 0.0000 & 0.0000 & 0.0000 & 0.0166 \\
0.0000 & 0.0000 & 0.0000 & 0.0431 & 0.0000 & 0.0000 & 0.0166 \\
0.0000 & 0.0000 & 0.0000 & 0.0000 & 0.0000 & 0.0148 & 0.0166
\end{array}\right]
$$

However, these are only 6 of the needed $9-3+1=7$ solution vectors which span the space. The remaining solution vector is produced from the nullspace vector for the non-zero values of the row:

$$
\left[\begin{array}{ll}
-0.8510 & -0.3430
\end{array}\right]
$$

which has a nullspace vector:

$$
\left[\begin{array}{ll}
-0.3738 & -0.9275
\end{array}\right]^{T}
$$

Next the nullspace vector is transposed, and the values of one solution are inserted in positions of the columns that did not contribute to the nullspace vector. For simplicity, values from the first solution are used producing the solution vector

$$
\left[\begin{array}{lllllllll}
0.0000 & 0.0000 & 0.0000 & 0.3738 & -0.9275 & 0.0000 & -0.0101 & 0.0000 & 0.0000
\end{array}\right]
$$

Next the solutions created by the reduced matrix are modified by inserting zero values in the positions of eliminated columns. Then the solution vectors created by SpecialCasel are appended to the end of them creating the final $m-n+1$ linearly independent solution vectors. 


$\left[\begin{array}{rrrrrrrrr}0.0000 & 0.0000 & 0.0000 & 0.0000 & 0.0000 & 0.0000 & -0.0101 & 0.0000 & 0.0000 \\ -0.0120 & 0.0000 & 0.0000 & 0.0000 & 0.0000 & 0.0000 & 0.0000 & 0.0000 & 0.0166 \\ 0.0000 & 0.0233 & 0.0000 & 0.0000 & 0.0000 & 0.0000 & 0.0000 & 0.0000 & 0.0166 \\ 0.0000 & 0.0000 & 0.0168 & 0.0000 & 0.0000 & 0.0000 & 0.0000 & 0.0000 & 0.0166 \\ 0.0000 & 0.0000 & 0.0000 & 0.0000 & 0.0000 & 0.0431 & 0.0000 & 0.0000 & 0.0166 \\ 0.0000 & 0.0000 & 0.0000 & 0.0000 & 0.0000 & 0.0000 & 0.0000 & 0.0148 & 0.0166 \\ 0.0000 & 0.0000 & 0.0000 & 0.3738 & -0.9275 & 0.0000 & -0.0101 & 0.0000 & 0.0000\end{array}\right]$

\subsection{SPECIALCASE2}

During testing of the algorithm yet another particular case, called SpecialCase2, caused difficulty finding the needed $m-n+1$ linearly independent solution vectors. This case, unlike SpecialCase1, occurs when all components of the $b$ vector are non-zero.

Write the equation $A \bar{x}=b$ as follows:

$$
\left[\begin{array}{cccc}
A_{11} & A_{12} & \cdots & A_{1 m} \\
A_{21} & A_{22} & \cdots & A_{2 m} \\
\vdots & \vdots & \ddots & \vdots \\
A_{n 1} & A_{n 2} & \cdots & A_{n m}
\end{array}\right] \bullet\left[\begin{array}{c}
X_{1} \\
X_{2} \\
\vdots \\
X_{m}
\end{array}\right]=\left[\begin{array}{c}
b_{1} \\
b_{2} \\
\vdots \\
b_{n}
\end{array}\right]
$$

Consider all the columns in $A$ being split into two categories. For simplicity the first $i$ columns fit under the Classification $\mathrm{A}$, and the remaining $m-i$ components will satisfy Classification $B$. Classification $A$ is that for all these columns they must have row multiplicity. For example, if these were the only columns in the matrix, the matrix would have a nullity equal to $n$. Classification B states that for all of the remaining columns not in the first $i$, at least two rows must satisfy the condition $A_{2_{q}} / A_{1_{q}}=b_{2} / b_{1}=\beta$ (again for simplicity the first two rows have been selected. Thus $A$ may be expressed as

$$
\left[\begin{array}{cccccccc}
A_{1 p_{1}} & A_{1 p_{2}} & \cdots & A_{1 p_{2}} & A_{1 q_{1}} & A_{1 q_{2}} & \cdots & A_{1 q_{m-i}} \\
A_{2 p_{1}} & A_{2 p_{2}} & \cdots & A_{2 p_{i}} & A_{2 q_{1}} & A_{2 q_{1}} & \cdots & A_{2 q_{m-i}} \\
\vdots & \vdots & \ddots & \vdots & \vdots & \vdots & \ddots & \vdots \\
A_{n p_{1}} & A_{n p_{2}} & \cdots & A_{n p_{i}} & A_{n q_{1}} & A_{n q_{2}} & \cdots & A_{n q_{m-i}}
\end{array}\right]
$$

with the property that $\left[A_{1 p_{1}} \ldots A_{1 p_{i}}\right]=C_{2}\left[A_{2 p_{1}} \ldots A_{2 p_{i}}\right]=C_{n}\left[A_{n p_{1}} \ldots A_{n p_{i}}\right]$ and $A_{2 q_{1}}=\beta A_{1 q_{1}}, A_{2 q_{2}}=\beta A_{1 q_{2}}, \ldots, A_{2 q_{(m-i)}}=\beta A_{1 q_{(m-i)}}$. 
Substituting $A$ from Eq. (10) into Eq. (9), the products from the first and second rows become Eqs. (11) and (12) respectively:

$$
\begin{gathered}
A_{1 p_{1}} X_{p_{1}}+A_{1 p_{2}} X_{p_{2}}+\cdots+A_{1 p_{1}} X_{p_{1}}+A_{1 q_{1}} X_{q_{1}}+A_{1 q_{2}} X_{q_{2}}+\cdots+A_{1 q_{m-1}} X_{q_{m-1}}=b_{1} \\
A_{2 p_{1}} X_{p_{1}}+A_{2 p_{2}} X_{p_{2}}+\cdots+A_{2 p_{1}} X_{p_{1}}+A_{2 p_{1}} X_{q_{1}}+A_{2 q_{2}} X_{q_{2}}+\cdots+A_{2 q_{m-1}} X_{q_{m-1}}=b_{2}
\end{gathered}
$$

Equation (12) can be reduced using the properties of Classifications A and B shown in Eq. (10), giving:

$$
\begin{aligned}
& C_{2}^{-1}\left(A_{1 p_{1}} X_{p_{1}}+A_{1 p_{2}} X_{p_{2}}+\cdots+A_{1 p_{i}} X_{p_{i}}\right)+\beta\left(A_{1 q_{1}} X_{q_{1}}+A_{1 q_{2}} X_{q_{2}}+\cdots+A_{1 q_{m-i}} X_{q_{m-1}}\right)=\beta b_{1} \\
& \left(\beta C_{2}\right)^{-1}\left(A_{1 p_{1}} X_{p_{1}}+A_{1 p_{2}} X_{p_{2}}+\cdots+A_{1 p_{1}} X_{p_{i}}\right)+A_{1 q_{1}} X_{q_{1}}+A_{1 q_{2}} X_{q_{2}}+\cdots+A_{1 q_{m-1}} X_{q_{m-1}}=b_{1}
\end{aligned}
$$

Next, $b_{1}$ in Eq. (13) can be substituted into Eq. (11). Using a new constant value $K=1-\left(\beta C_{2}\right)^{-1}$ the substitution reduces to

$$
\begin{gathered}
K\left(A_{1 p_{1}} X_{p_{1}}+A_{1 p_{2}} X_{p_{2}}+\cdots+A_{1 p_{i}} X_{p_{i}}\right)=0 \\
A_{1 p_{1}} X_{p_{1}}+A_{1 p_{2}} X_{p_{2}}+\cdots+A_{1 p_{1}} X_{p_{i}}=0
\end{gathered}
$$

Due to Classification $\mathrm{A}$, any row $k$ such that $k \neq 1$ can have its product reduced using Eq. (14):

$$
\begin{gathered}
C_{k}^{-1}\left(A_{1 p_{1}} X_{p_{1}}+A_{1 p_{2}} X_{p_{2}}+\cdots+A_{1 p_{i}} X_{p_{i}}\right)+A_{k q_{1}} X_{q_{1}}+A_{k q_{2}} X_{q_{2}}+\cdots+A_{k q_{m-1}} X_{q_{m-i}}=b_{n} \\
A_{k q_{1}} X_{q_{1}}+A_{k q_{2}} X_{q_{2}}+\cdots+A_{k q_{m-i}} X_{q_{m-i}}=b_{n}
\end{gathered}
$$

Using Eqs. (14) and (15), the original matrix $A$ in Eq. (10) can be reduced, creating the system

$$
\left[\begin{array}{cccccccc}
A_{1 p_{1}} & A_{1 p_{2}} & \cdots & A_{1 p_{i}} & 0 & 0 & \cdots & 0 \\
0 & 0 & \cdots & 0 & A_{2 q_{1}} & A_{2 q_{2}} & \cdots & A_{2 q_{m-i}} \\
\vdots & \vdots & \ddots & \vdots & \vdots & \vdots & \ddots & \vdots \\
0 & 0 & \cdots & 0 & A_{n q_{1}} & A_{n q_{2}} & \cdots & A_{n q_{m-i}}
\end{array}\right] \bullet\left[\begin{array}{c}
X_{1} \\
X_{2} \\
\vdots \\
X_{m}
\end{array}\right]=\left[\begin{array}{c}
b_{1} \\
b_{2} \\
\vdots \\
b_{n}
\end{array}\right]
$$


The reduced system in Eq. (16) can be seen as two systems: one containing the first $i$ degrees of freedom, and the second system consisting of the remaining degrees of freedom. Thus the first $i$ columns and first row may be eliminated so the second system may be solved. Next, the additional needed solution vectors can be gathered from the first subsystem, being the nullspace of the first row. This is done in the same way as in SpecialCasel. In this case, however, the columns that satisfy Classification A are removed rather than the nonzero ones as in SpecialCase1.

An example of a system which contains a SpecialCase2 is shown below. Notice that the first $i$ columns do not satisfy Classification $\mathrm{A}$, and the first and second row do not contain a SpecialCase2.

$$
\left[\begin{array}{llllll}
1 & 1 & 2 & 2 & 4 & 3 \\
2 & 5 & 4 & 0 & 9 & 6 \\
3 & 2 & 6 & 4 & 8 & 9
\end{array}\right] \cdot\left[\begin{array}{l}
X_{1} \\
X_{2} \\
X_{3} \\
X_{4} \\
X_{5} \\
X_{6}
\end{array}\right]=\left[\begin{array}{l}
3 \\
7 \\
6
\end{array}\right]
$$

In this system the SpecialCase 2 occurs in rows 1 and 3 with columns 1,3 , and 6 satisfying Classification $\mathrm{A}$. To detect such a case, the program follows a specific time saving algorithm. This algorithm checks all possible beta values by combining every combination of elements in the $b$ vector. For example, with the above system, the first beta created will be formed from rows 1 and 2 (equal to (7/3)). After calculating the beta the algorithm searches the rows of the Jacobian that formed the beta, column by column, for Classification B. The first values it checks are the 1 and 2 of column 1 which have a beta equal to 2 . Since the first column does not satisfy Classification $B$, it must satisfy Classification A for a SpecialCase2 to occur. For this reason column 1 is marked as being Classification A, and a new array is formed. The new array contains the constant multiples of every row in column 1 with respect to some reference position. For instance if the reference was the first position, and the first column was $\left[A_{11}, C_{2} A_{11}, \ldots, C_{N} A_{11}\right]^{T}$ then the array would contain $\left[1, C_{2}, \ldots, C_{N}\right]$. The reference value is simply one of the rows that formed the beta value that does not contain a zero value. If both of these positions contain a zero value, then the next possible beta may be checked because it is impossible for a SpecialCase2 to occur for such a situation unless there is a column of zeros present which has already been checked. For the example case the first reference chosen is row 1 , creating the array $[1,2,3]$.

After the reference has been chosen and the array has been formed, the next column is checked for Classification B. In our example it fails again (beta $=5$ ), so it is assumed to be Classification A. Using the same reference, this entire column is compared to the first 
Classification A column. This new Classification A column fails when the first position is compared, so the combination of rows 1 and 2 fails the SpecialCase 2 check.

Next rows 1 and 3 are compared which form a beta equal to 2 . The first column again fails Classification $B$ and thus again becomes Classification $A$ with reference one and retaining the same array. Next, column 2 is checked which has a beta equal to 2, so the algorithm moves to column 3 which has a beta equal to 3 . Thus column 3 is put into Classification $\mathrm{A}$ and compared to the current array and reference which does not fail this time. So columns 4 and 5 are checked having betas both equal to $2((4 / 2)$ and $(8 / 4))$ respectively), and finally column 6 which falls into Classification A. Now that the algorithm has checked all the columns finding that they all satisfy either the requirements for Classification A or B, it is determined that a SpecialCase2 is present. Such an instance stops all checking for SpecialCase 2 and begins reduction.

While checking, the program has kept track of both a reference row and all columns which satisfy Classification A. Thus, to reduce the matrix all the Classification A columns and the reference row are eliminated, producing the system:

$$
\left[\begin{array}{lll}
5 & 0 & 9 \\
2 & 4 & 8
\end{array}\right] \bullet\left[\begin{array}{c}
X_{2} \\
X_{4} \\
X_{5}
\end{array}\right]=\left[\begin{array}{l}
7 \\
6
\end{array}\right]
$$

This system can easily be handled by the FSP algorithm producing the solution vectors below, whose 1,3 , and 6 columns have been filled with zeros due to elimination.

$$
\left[\begin{array}{rrrrrr}
0.0000 & 1.4000 & 0.0000 & 0.8000 & 0.0000 & 0.0000 \\
0.0000 & 0.0000 & 0.0000 & -0.0556 & 0.7778 & 0.0000
\end{array}\right]
$$

However these are only two of the $(6-3+1)=4$ needed linearly independent solution vectors. As discussed before, the two remaining vectors come from the nullspace of the eliminated row and columns. Thus, these two vectors come from the nullspace of the vector [ 123$]$ and are

$$
\left[\begin{array}{rrr}
0.0000 & -0.8321 & 0.5546 \\
0.9636 & -0.1482 & -0.2224
\end{array}\right]
$$

These two nullspace vectors are added to the previous two solution vectors with one of the previous solution vector values in the place of non-Classification A positions. This is done so that the SpecialCase2 solution vectors satisfy both the reduced and original system. For ease, the first solution is used for this substitution, thus the following set of solution vectors is created: 


$\left[\begin{array}{rrrrrr}0.0000 & 1.4000 & 0.0000 & 0.8000 & 0.0000 & 0.0000 \\ 0.0000 & 0.0000 & 0.0000 & -0.0556 & 0.7778 & 0.0000 \\ 0.0000 & 1.4000 & -0.8321 & 0.8000 & 0.0000 & 0.5547 \\ 0.9636 & 1.4000 & -0.1482 & 0.8000 & 0.0000 & -0.2224\end{array}\right]$




\section{HANDLING A LOSS OF RANK}

A loss of row rank in the matrix $A$ is first detected by the reduction procedure. To indicate such a case it has been stated that a value of " 2 " is stored in RowElim in the position of the row which has been eliminated due to the dependency. When FSPv2.0 has completed, and the Mred-Nred +1 solutions are generated, a check is performed to see if such a rank loss has occurred before going on to find a solution for $x$. If $r$ rows have been eliminated due to such a case, there would be $m-n+1+r$ linearly independent solution vectors created by FSPv2.0, but it should also be noted that the elimination of $r$ rows has created $r$ constraints on the system. Thus the solution space retains its original dimension and can be written as

$$
S=\left\{x \mid x=\sum_{i=1}^{m-n+1+r} t_{i} g^{i}, \quad \sum_{k=1}^{m-n+1+r} t_{k}=1, \quad \sum_{i=1}^{m-n+1+r} \beta_{i}^{j} t_{i}=1 ; j=1, r\right\}
$$

In Eq. (17) the terminology is the same that has been used in [1,3,4]. Each solution vector is expressed as $g^{i}$, and $\bar{t}$ is a vector of constants whose sum equals one [3]. The vectors $\beta^{j}$ represent the constraints imposed due to a loss of rank with each constraint written as:

$$
\beta_{i}^{j}=\frac{<A_{\ell} g^{i}>}{b_{\ell}}
$$

In Eq. (18), $A_{\ell}$ represents the dependent row vector which the current beta is being created for.

The major change in FSPv2.0 is the incorporation of the creation of these constraints in the system. Thus, after creating the solution vectors, FSPv2.0 checks to see if the matrix has lost rank. If such a case occurs, it calls a procedure which creates the constraint vector according to Eq. (18). Another feature of FSPv2.0 is its ability to handle a multiple rank lost situation. For such a case, the procedure is called multiple times in order to create more than one constraint. 


\section{EXAMPLES OF REDUCTION}

\subsection{ENDURANCE TEST}

The following system is an unrealistic system but was designed to show the operation of the reduction algorithm in great detail. To show this, the following example has a column of zeros, a SpecialCase1 which creates a SpecialCase2 which then creates a restricted element. The system is

$$
\left[\begin{array}{llllllll}
1 & 0 & 0 & 0 & 0 & 1 & 0 & 0 \\
0 & 3 & 1 & 0 & 2 & 0 & 1 & 4 \\
0 & 6 & 0 & 0 & 4 & 0 & 0 & 8 \\
0 & 5 & 6 & 0 & 0 & 0 & 6 & 0
\end{array}\right] \cdot\left[\begin{array}{c}
X_{1} \\
X_{2} \\
X_{3} \\
X_{4} \\
X_{5} \\
X_{6} \\
X_{7} \\
X_{8}
\end{array}\right]=\left[\begin{array}{l}
0 \\
1 \\
2 \\
3
\end{array}\right]
$$

First, the algorithm checks for any rows with less than two non-zero elements in it. After this is done it next checks columns of all zero values, which column 4 satisfies, then checks for row dependency. Thus after a first check for common occurrences, the matrix $A$ effectively becomes

$$
\begin{aligned}
& \text { Column \# } \\
& \begin{array}{|l|l|l|l|l|l|l|}
\hline 1 & 2 & 3 & 5 & 6 & 7 & 8 \\
\hline
\end{array} \\
& \text { Row \# }\left[\begin{array}{lllllll}
1 & 1 \\
2 \\
2 \\
3 \\
4
\end{array}\right]\left[\begin{array}{lllllll}
1 & 0 & 0 & 0 & 1 & 0 & 0 \\
0 & 3 & 1 & 2 & 0 & 1 & 4 \\
0 & 6 & 0 & 4 & 0 & 0 & 8 \\
0 & 5 & 6 & 0 & 0 & 6 & 0
\end{array}\right]
\end{aligned}
$$

Now, the check for special cases begin with a check for SpecialCase1. A's can be seen above, the $b$ vector contains only one zero element, corresponding to the first row. Thus all non-zero elements in row one of the current matrix $A$ are considered. The first element found is in column 1, and the second and final value is found in column 6 . Next 
these columns are tested for multiplicity (see Section 5). Since they satisfy the test, they are determined to cause a SpecialCase1, and the corresponding row and columns are eliminated from $A$. To create the additional solution vector, the nullspace of the vector $[1,1]$ is found, and the solution vector $[0.7071,0,0,0,0,-0.7071,0,0]$ is created.

Since a SpecialCase1 has been detected and removed, the system must check again for any common occurrences. The new system now is

$$
\begin{gathered}
\text { Column \# } \\
\left.\begin{array}{llllll}
2 & 3 & 5 & 7 & 8
\end{array}\right] \\
\text { Row \#[ } \frac{2}{3}\left[\begin{array}{lllll}
3 & 1 & 2 & 1 & 4 \\
6 & 0 & 4 & 0 & 8 \\
5 & 6 & 0 & 6 & 0
\end{array}\right] \cdot \bar{x}=\left[\begin{array}{l}
1 \\
2 \\
3
\end{array}\right]
\end{gathered}
$$

One can see that the newly created matrix $A$ has no common occurrences and does not contain a SpecialCasel due to the $b$ vector being entirely composed of non-zero values. Thus the algorithm, in the second iteration, moves on to check for a SpecialCase2. Such a case can be easily recognized in rows 2 and 3 with $\beta=2$, ClassificationA columns 3 and 7 , and ClassificationB columns 2, 5, and 8. Therefore the program removes row 2 and columns 3 and 7 creating the new solution vector $[0,0,0.7071,0,0,0,-0.7071,0]$.

The occurrence of another special case has created the need to completely check the reduction loop again sending it the new system

$$
\begin{gathered}
\text { Column \# } \\
\begin{array}{llll}
\hline 2 & 5 & 8 \\
\text { Row \# }
\end{array} \\
\hline 4\left[\begin{array}{lll}
6 & 4 & 8 \\
5 & 0 & 0
\end{array}\right] \cdot \bar{x}=\left[\begin{array}{l}
2 \\
3
\end{array}\right]
\end{gathered}
$$

The new reduced system is again checked for restrictions which it contains in row 4 . To handle such a case, it fixes the value $X_{2}=(3 / 5)=0.6$; eliminates row 4 and column 2, and backsubstitutes the remaining values of column 2 into the $b$ vector. These computations create the new following system 


\section{RUN TIME TESTING}

To compare the two separate FSP algorithms, tests were done on the unchanged FSPv1.0 and the FSPv2.0 code without special case checks. This method was chosen so that the two versions could handle all of the same cases and had the same reduction procedure including the existence of the occasional misrecognition of dependent rows (see Fig. 3 in Section 2). Therefore only the initial block selection and remaining solution generators were effectively compared between the two versions, keeping the method of reduction constant. In addition, to further increase accuracy of measurement, all debugging statements in each version were shut off and each version was driven by the same code. Data for each test were collected from random trajectories which consisted of 170 to 330 time steps. Next the completion time for each time step was computed from total computing time of each trajectory, creating an average value for each time step in that trajectory. To show the relative speed of each version, a standard pseudo-inverse algorithm (found in Ref. 4) was used so that this system could be compared with other various systems.

The first manipulator arm used to compare the two versions was the HERMIES arm with a holonomic platform. The data for this configuration can be seen in Fig. 10 below. One aspect to notice is that the completion time of FSPv2.0 is faster than FSPv1 .0 by about 15 percent. This difference is also apparent in Table 1 which shows the comparison ratios of each version to the pseudo-inverse method.

\section{Runtime Comparison of FSPv1.0, FSPv2.0, and Pseudo-Inverse for the HERMIES Configuration}

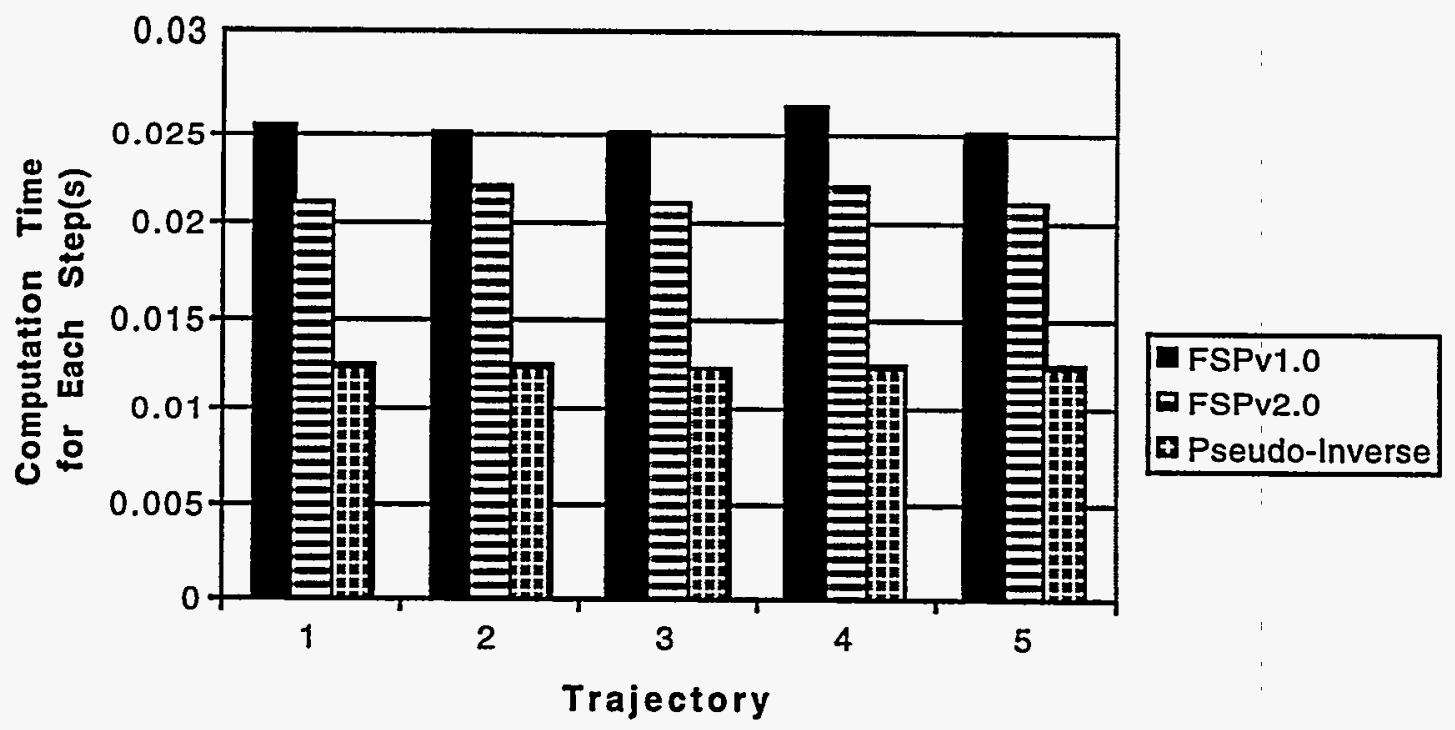

Fig. 10. Timing comparison for the HERMIES manipulator arm. 
$\because$ 


$$
\begin{aligned}
& \text { Column \# } \\
& \begin{array}{l|l|l}
5 & 8
\end{array} \\
& \text { Row \# } 3\left[\begin{array}{ll}
4 & 8
\end{array}\right] \cdot \overline{X^{\prime}}=[-1.6]
\end{aligned}
$$

Next the checks for row dependency, zero columns, and special cases are done. After the program has deduced that such a circumstance does not exist, it proceeds to generate the additional two solution vectors for the original system.

\subsection{MULTIPLE RANK LOST}

For a matrix that has multiple dependent rows, the procedure eliminates the rows which create the problem. In addition, to eliminating such rows, the procedure marks them so that a constraint will be created for the elimination. An example of such a problematic $A$ is shown below.

$$
\left[\begin{array}{lllll}
1 & 1 & 1 & 1 & 1 \\
2 & 2 & 2 & 2 & 2 \\
1 & 0 & 1 & 0 & 1 \\
3 & 3 & 3 & 3 & 3 \\
0 & 1 & 0 & 1 & 0
\end{array}\right]
$$

For this matrix, the program transposes the matrix and finds the nullity of the matrix which equals two. Then it searches through these nullspace vectors to find which rows contribute to each one. Then it eliminates one row in the first vector and one different row in the second vector which produces the reduced $A$ equal to

$$
\left[\begin{array}{lllll}
1 & 0 & 1 & 0 & 1 \\
3 & 3 & 3 & 3 & 3 \\
0 & 1 & 0 & 1 & 0
\end{array}\right]
$$


Table 1. Ratio of computation time of FSPv1.0 and FSPv2.0 to pseudo-inverse for the HERMIES configuration

\begin{tabular}{|c|c|c|c|c|c|}
\hline Trajectory & 1 & 2 & 3 & 4 & 5 \\
\hline FSPv1.0 & 2.08 & 2.07 & 2.13 & 2.14 & 2.04 \\
\hline FSPv2.0 & 1.77 & 1.80 & 1.81 & 1.81 & 1.77 \\
\hline
\end{tabular}

To further compare the versions, the AIRARM with holonomic platform configuration was also used with the same five random trajectories. One major problem called for modification of the testing procedure. Due to the nature of the Jacobian matrices produced by the AIRARM configuration, the system is prone to the misrecognition of dependent rows error described in Section 2. To circumvent such a case, the method for solving this problem was placed in the reduction procedure of both FSPv1.0 and FSPv2.0. The collected data is shown in Fig. 11 which again shows that FSPv2.0 is 15 percent faster than FSPv1.0. In addition, the completion times for all methods are decreased due to the nature of the manipulator which has only 5 degrees of redundancy rather than the 7 in the HERMIES system.

- Runtime Comparison of FSPv1.0, FSPv2.0, and Pseudo-Inverse for the AIRARM Configuration

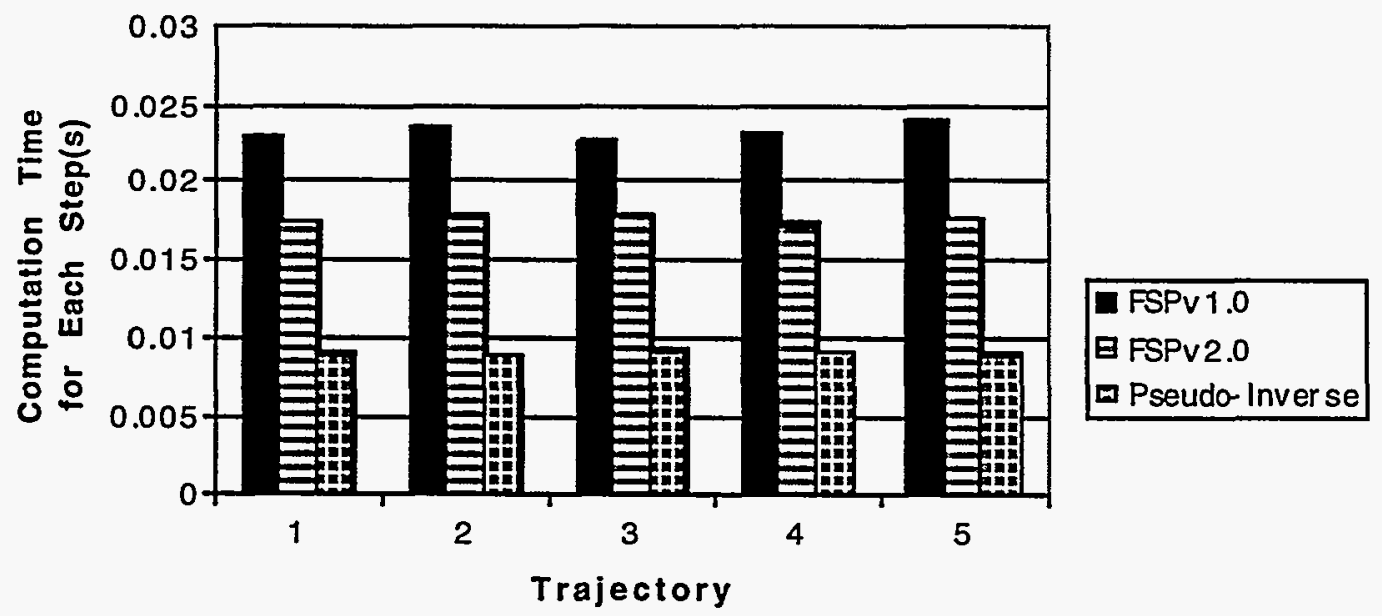

Fig. 11. Timing comparison for the AIRARM manipulator arm.

Table 2. Ratio of computation time of FSPv1.0 and FSPv2.0 to pseudo-inverse for the AIRARM configuration

\begin{tabular}{|c|c|c|c|c|c|}
\hline Trajectory & $\mathbf{1}$ & $\mathbf{2}$ & $\mathbf{3}$ & $\mathbf{4}$ & $\mathbf{5}$ \\
\hline FSPv1.0 & 2.56 & 2.65 & 2.48 & 2.55 & 2.70 \\
\hline FSPv2.0 & 1.93 & 2.00 & 1.95 & 1.91 & 1.99 \\
\hline
\end{tabular}


Each randomly created trajectory produced a set of systems which each version attempted to solve. Two separate manipulator configurations were used to show how the versions compared to one another for different setups. Table 3 summaries the results of the two configurations with the ratio of completion time between the two versions.

Table 3. Ratio of computation time; FSPv1.0 to FSPv2.0

\begin{tabular}{|c|c|c|c|c|c|}
\hline Trajectory & 1 & 2 & 3 & 4 & 5 \\
\hline HERMIES & 1.18 & 1.15 & 1.18 & 1.18 & 1.15 \\
\hline AIRARM & 1.32 & 1.33 & 1.27 & 1.33 & 1.36 \\
\hline
\end{tabular}

Variations in the ratio for each configuration are mainly due to fluctuations in FSPv1.0 where a completely new first solution is needed, and the searching must be started over again for that time step. Trajectories which contain many such cases would thus cause a larger average time step. This contrasts to the nearly constant average time for FSPv2.0 for each configuration because it does not need to restart the entire search. It can also be seen that the efficiency of FSPv2.0 significantly increases when the degree of redundancy is decreased. For pictures of trajectories and a complete listing of trajectory data see Appendix $C$ and Appendix D.

The next set of timing tests involved orientation control in addition to end-effector position control. With such a situation, the degree of redundancy is decreased by three. Thus for the HERMIES configuration there are only 4 degrees of redundancy, and for the AIRARM configuration there are only 2 degrees of redundancy. For such a case, one would assume that the computation time would be decreased considerably, but there is still another consideration other than number of branches. For a manipulator with orientation control each submatrix is $6 \times 6$ which consumes a much greater amount of computing time to invert compared to the previous $3 \times 3$ submatrix. Thus there is a trade-off between the decrease of time due to the decrease in the number of levels of branches to be searched and the increase in time due to a larger submatrix to invert.

A case which sways this trade-off to take more time is in the HERMIES configuration with holonomic platform. The timing results are shown in Fig. 12 and Table 4. From these graphs it can be seen that FSPv1.0 is very erratic during Trajectories 3 and 4. Again this can be attributed to the search pattern searching for a new first solution many times for most of the time steps in these trajectories. In addition it can be seen that FSPv2.0 again is quicker than FSPv1.0 by about 15 to 20 percent on average.

Table 4. Ratio of computation time of FSPv1.0 and FSPv2.0 to pseudo-inverse for the HERMIES configuration with orientation control

\begin{tabular}{|c|c|c|c|c|c|}
\hline Trajectory & $\mathbf{1}$ & $\mathbf{2}$ & 3 & 4 & 5 \\
\hline FSPv1.0 & 3.55 & 3.42 & 6.33 & 6.37 & 3.55 \\
\hline FSPv2.0 & 3.18 & 2.99 & 3.06 & 3.18 & 3.10 \\
\hline
\end{tabular}


Runtime Comparison of FSPv1.0, FSPv2.0, and Pseudo-Inverse for the AIRARM Configuration with Orientation Control

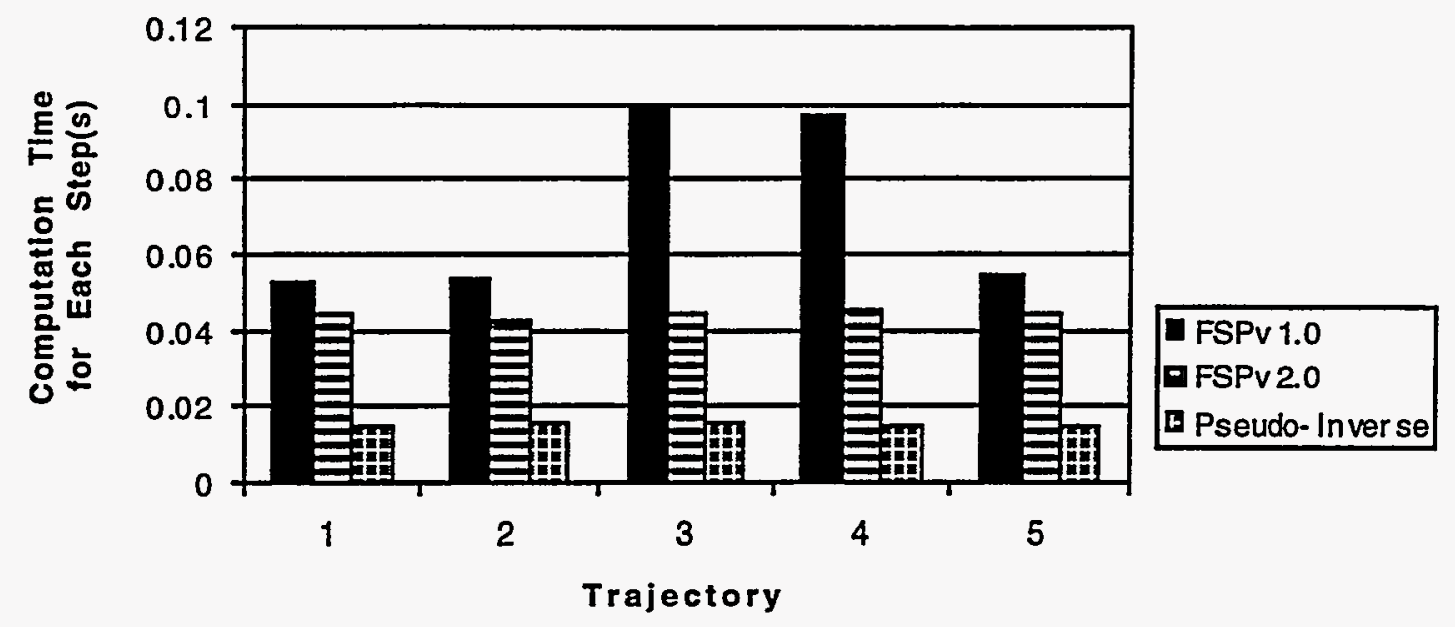

Fig. 12. Timing comparison for the AIRARM manipulator arm.

When orientation control is implemented on the AIRARM configuration, it can be seen that the previously mentioned balance remains at the same level. Figure 13 and Table 5 show the results of timing tests for such a scenario. The results of the test are close to the results without orientation control except for FSPv1.0 consuming more time, taking 20 to 30 percent longer than FSPv2.0.

Runtime Comparison of FSPv1.0, FSPv2.0, and Pseudo-Inverse for the AIRARM Configuration

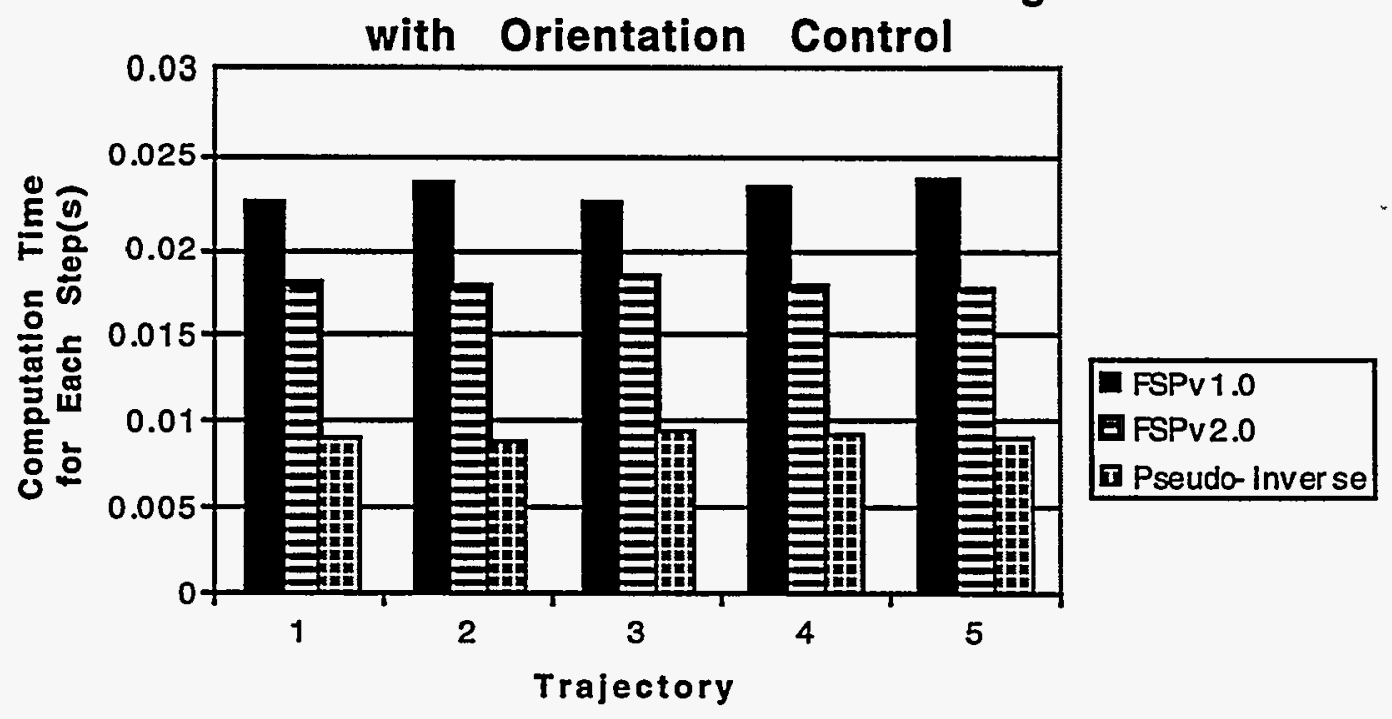

Fig. 13. Timing comparison for AIRARM manipulator arm. 
Table 5. Ratio of computation time of FSPv1.0 and FSPv2.0 to pseudo-inverse for the AIRARM configuration with orientation control

\begin{tabular}{|c|c|c|c|c|c|}
\hline Trajectory & 1 & 2 & 3 & 4 & 5 \\
\hline FSPv1.0 & 2.50 & 2.65 & 2.42 & 2.55 & 2.65 \\
\hline FSPv2.0 & 2.00 & 2.00 & 2.01 & 1.96 & 1.99 \\
\hline
\end{tabular}

When comparing FSPv1.0 and FSPv2.0 directly, the increase in code efficiency and consistency can be seen. Table 6 shows the results of such a comparison when orientation control is used.

Table 6. Ratio of computation time; FSPv1.0 to FSPv2.0, with orientation control

\begin{tabular}{|c|c|c|c|c|c|}
\hline Trajectory & 1 & 2 & 3 & 4 & 5 \\
\hline HERMIES & 1.12 & 1.14 & 2.07 & 1.99 & 1.15 \\
\hline AIRARM & 1.25 & 1.33 & 1.21 & 1.30 & 1.33 \\
\hline
\end{tabular}




\section{CONCLUSION}

This article discusses the enhancements and modifications made to the initial code for the recently developed Full Space Parameterization (FSP) method. It has been shown how the needed $m-n+1$ linearly independent solution vectors are determined. This method uses the branch-like structure of solution combinations so that large blocks can be eliminated while still considering all possible combinations. Timing tests were done on this new method comparing it to both FSPv1.0 and the pseudo-inverse method. It has been shown that this new method is on the order of 15 to 20 percent faster than FSPv1.0 and takes about 1.8 times as long as pseudo-inverse method.

In addition, the new code is able to handle special particular cases which were not considered in earlier versions. The methods for identifying and resolving these cases are outlined in detail. A listing of the code for this new method, incorporating the tests and reduction algorithms, is given in Appendix $B$.

The major time-using segments in FSPv2.0 are the nullity checks for a first solution and dependency checks which both use SVD. If a faster alternative method were used to calculate the nullspace of a matrix, the computing time would be reduced considerably. 


\section{ACKNOWLEDGMENT}

This research was supported in part by the U.S. Air Force Combat Command (ACC), the U.S. Air Force Munition Material Handling Equipment (MMHE) Focal Point, the U.S. Air Force Reliability and Maintainability Technology Insertion Program (PRAM-RAMTIP), the U.S. Department of Defense Office of the Secretary of Defense (OSD), and the U.S. Advanced Project Research Agency (ARPA), under Interagency Agreement 2146-H055-A1 between the U.S. Air Force Material Command (AFMC) San Antonio Air Logistics Center, Robotics and Automation Center of Excellence (SA/ALC-RACE) and the U.S. Department of Energy. 


\section{REFERENCES}

[1] K. A. Morgansen and F. G. Pin, "Enhanced Code for the Full Space Parameterization Approach to Solving Underspecified Systems of Algebraic Equations, V.1.0," Oak Ridge National Laboratory Technical Report No. ORNL/TM-12816, March 1995.

[2] B. Siciliano, "Kinematic Control of Redundant Robot Manipulators: A Tutorial," Journal of Intelligent and Robotic Systems 3, 201-212, 1990.

[3] F. G. Pin et al., "A New Solution Method for the Inverse Kinematics Joint Velocity Calculations of Redundant Manipulators," IEEE Conference on Robotics and Automation, San Diego, California, 96-102, 1994.

[4] W. H. Press, S. A. Teukolsky, W. T. Velterling, and B. P. Flannery, Numerical Recipes in C, Cambridge University Press, second edition, 1992.

[5] C. J. Hacker and F. G. Pin, "Inverse Kinematics on Redundant Systems, IKOR Driver (V.1.0 and V.2.0): FSP with Orientation Control, Platform Mobility, and Portability," Oak Ridge National Laboratory Technical Report No. ORNL/TM-13096 (in press). 


\section{APPENDIX A USERS GUIDE}

The current FSPv2.0 code can be used in both a one step and multiple step technique. The one step method is used when an individual equation in the form of (1) needs to be solved. This is particularly useful when analyzing special cases or when a solution for a single configuration and displacement is sought. The multiple step option is currently implemented as part of the IKOR system [5]. In it, a file of positions and orientations for each time step is sent to the system. Depending on the robot configuration, the system develops the Jacobian and displacements in both the position and orientation. For a complete guide for using this system see Ref. 5 .

The one step option was developed in response to the discovery of the particular cases outlined in this paper. What developed was a need to test FSP for a system of equations which has a very small chance of appearing in a created trajectory. Thus the one step option allows the user to design a system to analyze the performance of FSP without the entire IKOR system.

To implement a particular matrix the entire IKOR system must be run, compiling it with the oneshot macro active (see Ref. 5). Next a text file must be made which contains the Jacobian matrix with elements separated by spaces, then a carriage return, then the $d x$ in the same fashion. A sample of such a file is listed below.

$\begin{array}{cccccc}0 & 0 & 2 & 1 & 0 & 0 \\ 2 & 1 & 0 & 1 & 0 & 1 \\ 3 & 4 & 0 & 0 & 5 & 0 \\ & & & & & \\ -0.25 & -0.50 & 0.00\end{array}$




\section{APPENDIX B CODE LISTINGS}




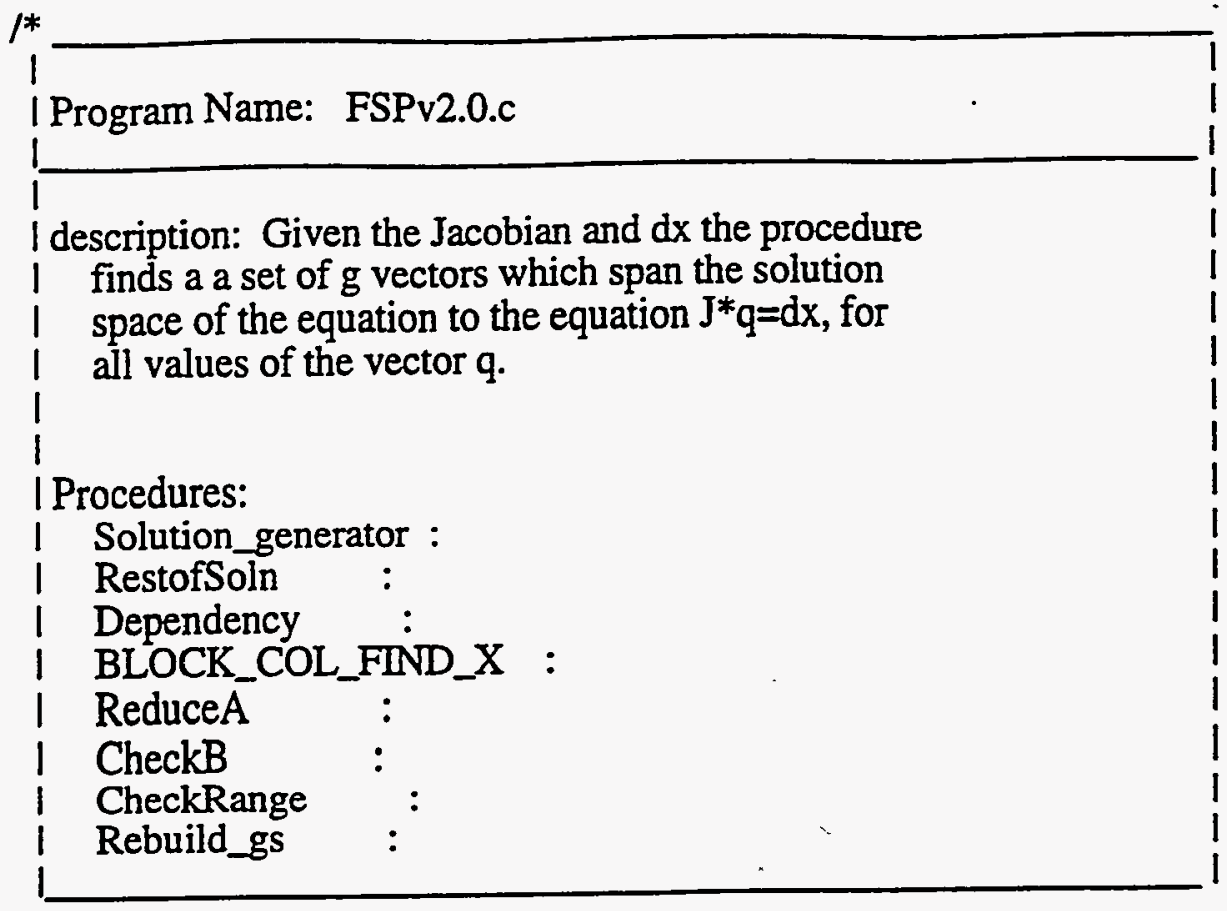

\#include "general.h"

int SystemComplete; /* marks when all necessary vecs have been found*I

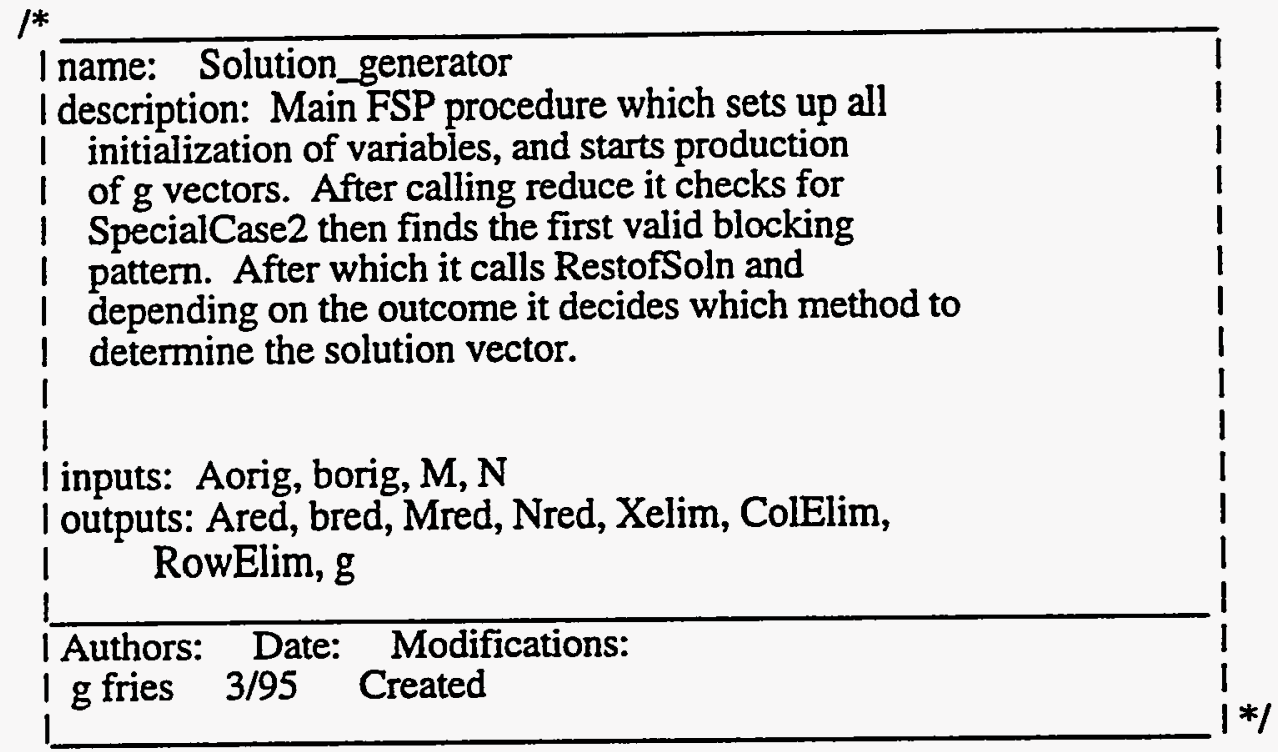

int Solution_generator(FSP_data, Aorig, borig, check)

FILE *check;

MATRIX *Aorig,

*borig;

Solutions *FSP_data;

MATRIX

*Ared, $\quad / *$ reduced A

*Asub, $\quad 1 *$ submatrix from square submatrix is found 
*Asqr, $\quad$ * square submatrix - - *I

*bred, $\quad 1 *$ reduced b *1

*block, $\quad /$ * locations of blocked columns for each soln *I

*Xelim, $\quad$ * comps of final $\mathrm{X}$ that were elim. in reduction */

$*_{n}, \quad / *$ null space vectors

*Specialg; $\quad$ * null vectors created by SpecialCase1 */

float

$\mathrm{K} 2, \quad \quad$ * matrix condition number: sv_max/sv_min

N_BND; $\quad$ * min acceptable value for nonzero nspace comp

int

$\mathrm{i}, \mathrm{j}, \mathrm{k}, \mathrm{I}, \quad / *$ loop counters

bcheck, $\quad t^{*}$ check for completely zero bred

*ColElim, $\quad$ * marks columns eliminated from original A

*RowElim, $1 *$ which rows are to be eliminated from the A

move, $\quad 1^{*}$ which of four possible blocks is being moved

NextToFind, /* vector number being searched for

*Tackon, $\quad / *$ columns are indexed for effic, soln finding

*FirstOK, $\quad 1 *$ if OK to substitute in for firstsoln column

pos, $\quad$ * used with the Ordering[ variable

nullity, $\quad \quad^{*}$ dimension of null space

binrange, $\quad \quad^{*}$ marks if $\mathrm{b}$ is in the range of the $\mathrm{A}$

Exit_Status, $/ *$ tells calling routine if $\mathrm{OK}$

NumSpg, $\quad l^{*}$ number of $g$ vectors created by SpecialCase 1

Stop; $\quad 1 *$ loop flag to end

I* allocate memory space for variables */

Tackon $=($ int $*)$ malloc $((M-N) *$ sizeof $($ int $))$;

FirstOK $=($ int $*$ ) malloc ( $\mathrm{N} *$ sizeof (int $)$ );

ColElim $=($ int $*)$ malloc $(M *$ sizeof $($ int $))$;

RowElim $=($ int $*)$ malloc $(N *$ sizeof $($ int $))$;

Ared = mat_malloc $(\mathrm{N}, \mathrm{M})$;

bred = mat_malloc $(\mathrm{N}, 1)$;

Xelim = mat_malloc(M,1);

Specialg= mat_malloc $((M-1), M)$;

/* initialize SystemComplete and FirstOK */

SystemComplete $=$ FALSE;

for $(i=0 ; i<N ; i++)$

FirstOK $[i]=$ FALSE;

$I^{*}$ check $=$ stderr; $\quad I^{*}$ uncomment when in trouble

$/ *$ Eliminate all the nonredundancies from the $A$ and $b * /$

ReduceA(FSP_data, Aorig, Ared, borig, bred, Xelim,ColElim,RowElim, \&NumSpg, Specialg);

/* Setup and initialization of $\mathrm{g}$ vectors */

mat_free(FSP_data->g);

FSP_data- $>$ g $=$ mat_malloc $($ FSP_data $>$ Mred-FSP_data- $>$ Nred $+1+N u m S p g), M)$;

for $(i=0 ; i<(S P A N) ; i++)$

for $(j=0 ; j<M ; j++)$

FSP_data- $>\mathrm{g}->\mathrm{p}[\mathrm{i}][\mathrm{i}]=0.0 \mathrm{e} 0$;

n = mat_malloc(FSP_data->Mred, FSP_data->Nred); 


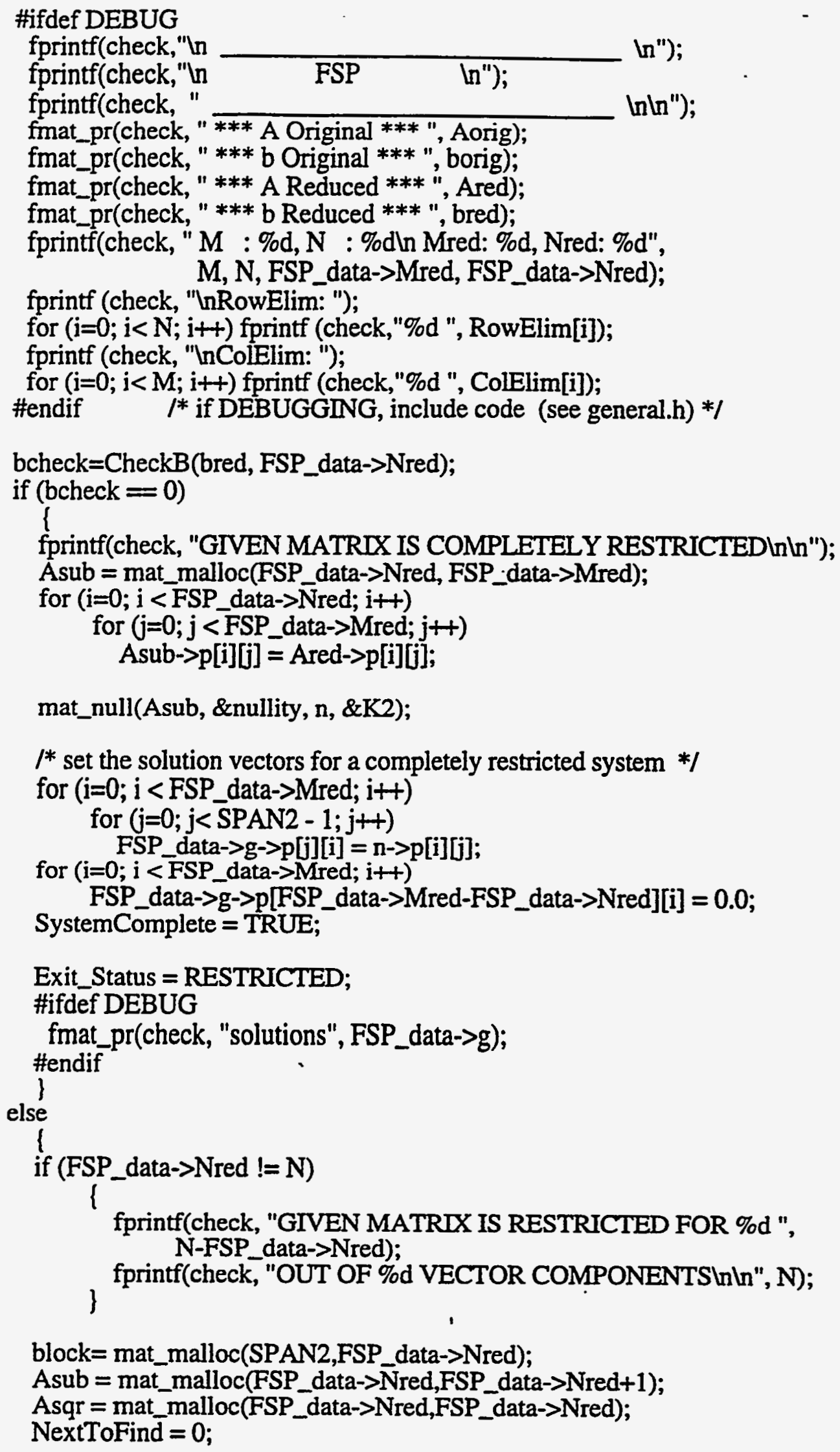


$/$ initialize the blocking positions for the first solution vector */ \#ifdef DEBUG

for $(\mathrm{i}=0 ; \mathrm{i}<$ (FSP_data->Nred); $\mathrm{i}++)$

for $(j=0 ; j<($ FSP_data- $>$ Mred-FSP_data- $>N r e d+1) ; j++)$

\#endif

$$
\text { block }->\mathrm{p}[\mathrm{j}][\overline{\mathrm{i}}]=0 \text {; }
$$

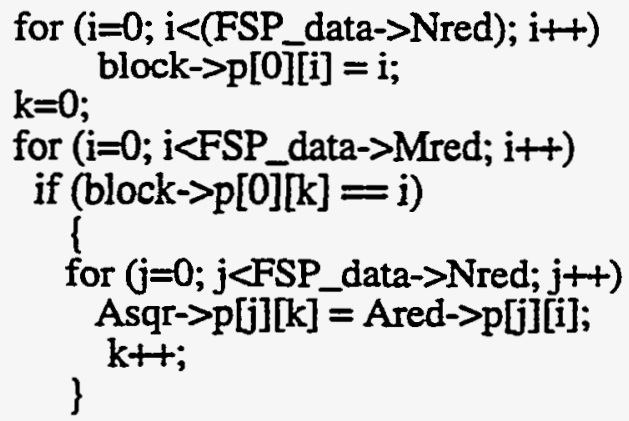

mat_null(Asqr, \&nullity, n, \&K2);

\#ifdef DEBUG

fmat_pr (check, "block", block);

fmat_pr (check, "Asqr", Asqr);

fprintf(check,"nullity = \%d, K2 = \%fln", nullity, K2);

\#endif $/ *$ if DEBUGGING, include code (see general.h) */

$1 *$ loop until an acceptable well-conditioned first solution found */

while ((nullity $!=0) \& \&$ (block->p $[0][0]<N)$ )

\{

pos = FSP_data $->$ Nred -1 ;

while (block->p[0][pos] $=$ SPAN2 - 1 +pos)

pos--;

block->p[0][pos]++;

move $=$ pos +1

while (move $\left.<F S P \_d a t a->N r e d\right)$

\{

block->p[0][move $]=$ block->p[0][(move- 1$)]+1$;

movet+;

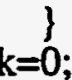

for $(\mathrm{i}=0$; i<FSP_data->Mred; $\mathrm{i}++)$

if (block->p[0] $[\mathrm{k}]=\mathrm{i}$ )

I

for $\left(j=0 ; j<F S P \_d a t a->N r e d ; j++\right)$

Asq $1->\mathrm{p}[\mathrm{j}][\mathrm{k}]=$ Ared $\rightarrow \mathrm{p}[\mathrm{i}][\mathrm{i}]$;

$\mathrm{k}++$;

\}

if (block $\rightarrow$ p [0][0] $<=$ FSP_data->Nred)

mat_null(Asqr, \&nullity, n, \& K2)!

\#ifdef DEBUG

fmat_pr(check, "block", block);

fmat_pr(check, "Asqr", Asqr);

fprintf(check,"nullity = \%d, K2 = \%fln", nullity, K2);

\#endif 


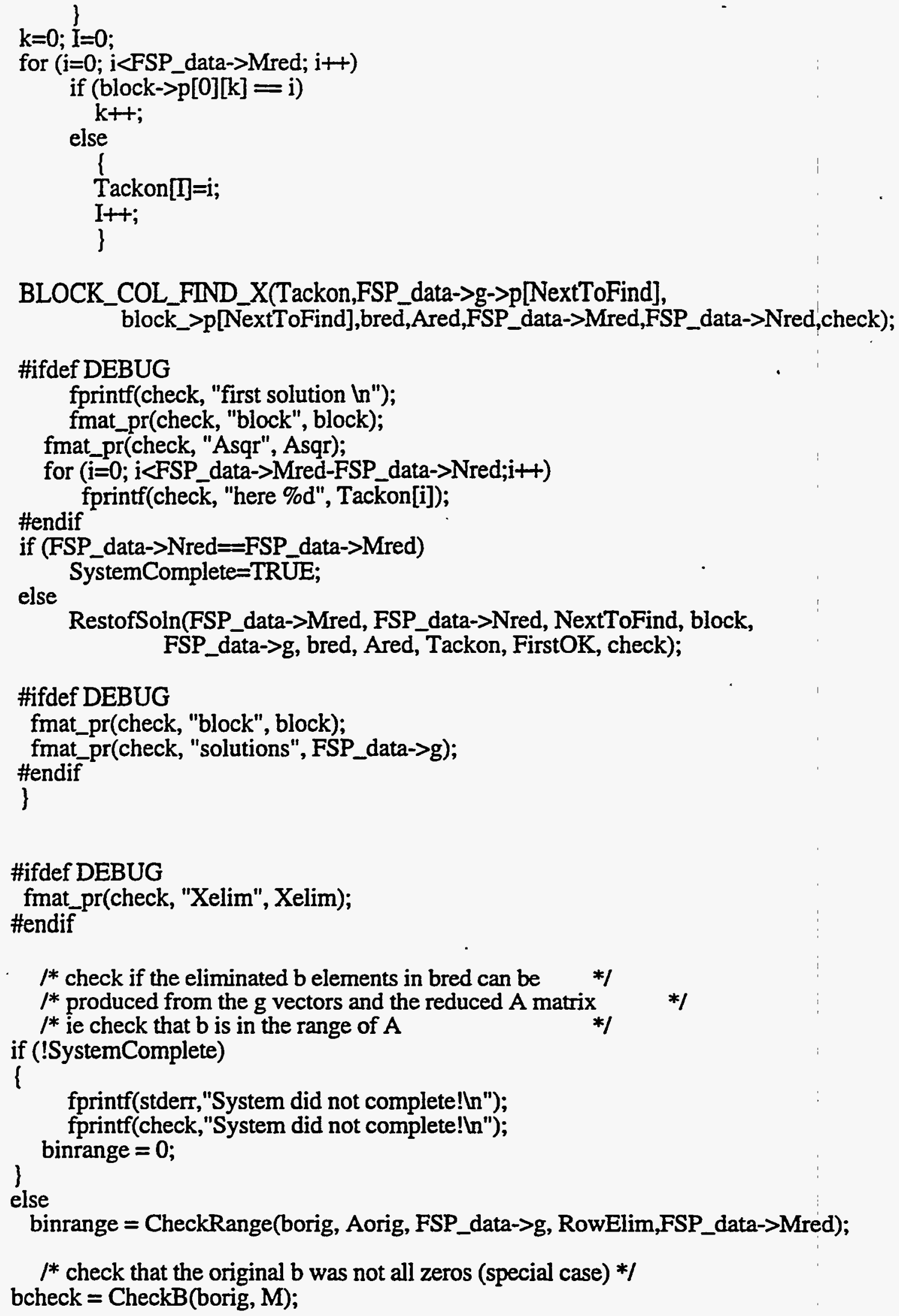

RestofSoln(FSP_data->Mred, FSP_data->Nred, NextToFind, block, FSP_data->g, bred, Ared, Tackon, FirstOK, check); 


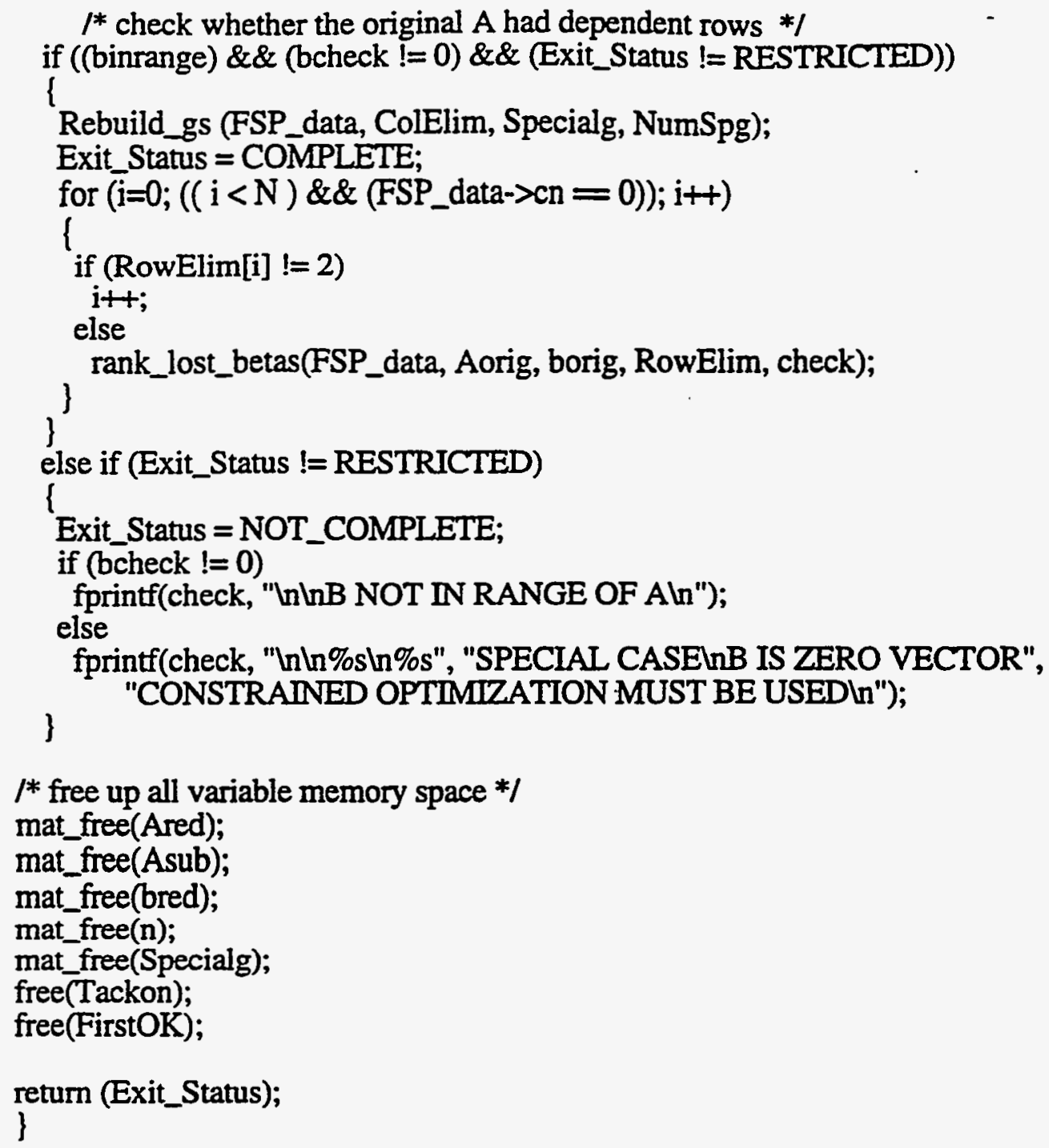



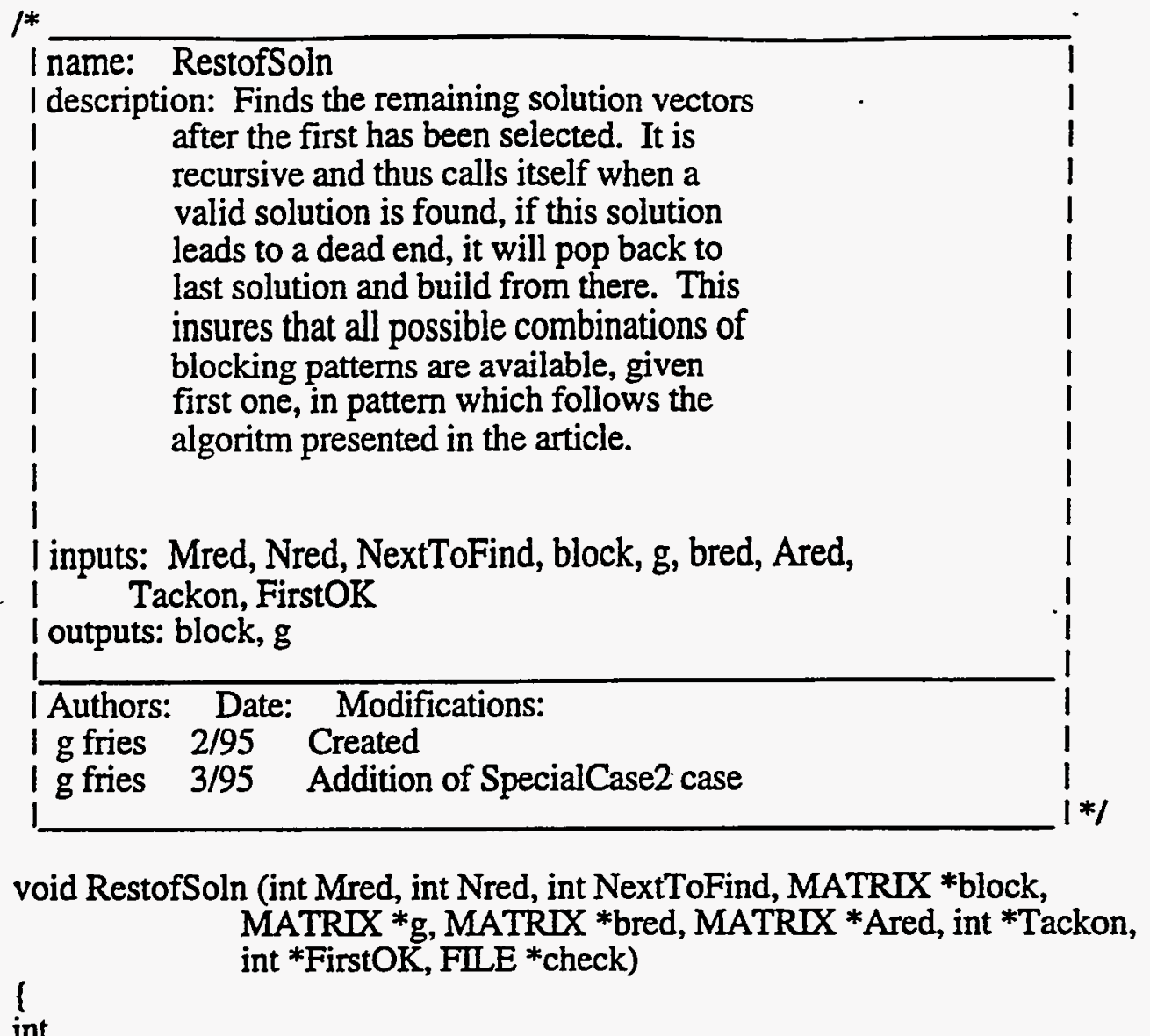


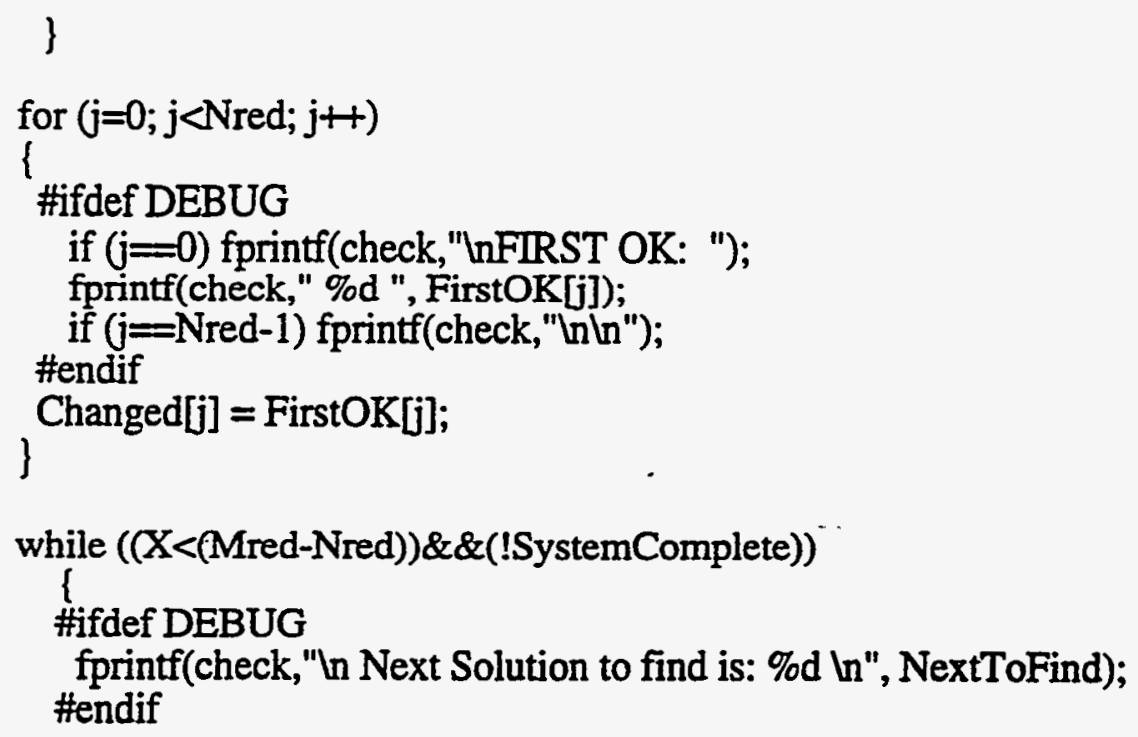

$1 *$ Set up next asub comparing the next remaining column to be subbed *I

$1 *$ in with the blocking pattern for that level of recursion */

Asub = mat_malloc(Nred,Nred +1 );

for $(\mathrm{i}=0 ; \mathrm{i}<\overline{\mathrm{N}} \mathrm{red} ; \mathrm{i}++)$

for $(j=0 ; j<N r e d ; j++)$

Asub->p[j][i] = Ared->p[j][(int)block->p[(NextToFind-1)][i]];

for $(j=0 ; j<N r e d ; j++)$

Asub $\rightarrow \mathrm{p}[\mathrm{j}][$ Nred $]=$ Ared->p[j][Tackon[X]];

\#ifdef DEBUG

fmat_pr(check, "Asub", Asub);

\#endif

$\mathrm{n}=$ mat_malloc(Mred,Nred);

mat_null(Asub, \&nullity, n, \&K2);

\#ifdef DEBUG

fmat_pr(check, "n", n);

\#endif

$\mathrm{Y}=0$;

/*Loop which compares column to be subbed in with current blocking *I

$/ *$ pattern for dependency, if dependent it completes swap and valid $* I$

while $((\mathrm{Y}<($ Nred $)) \& \&($ !SystemComplete $))$

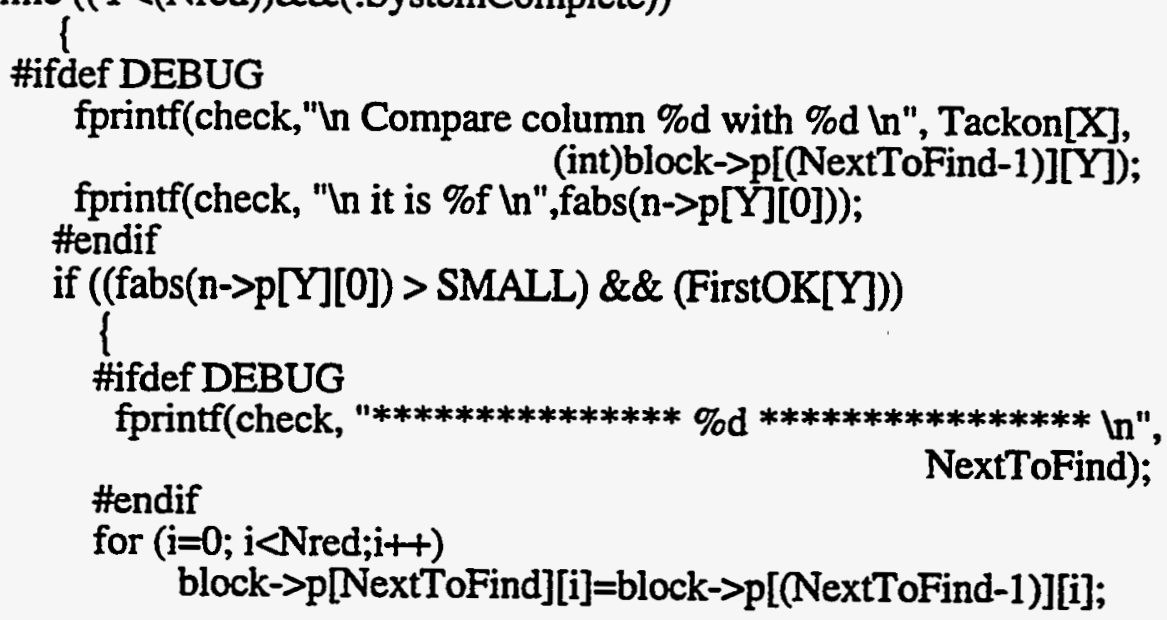


Newtack[0]=(int)block->p[NextToFind $][Y]$;

block->p[NextToFind $][\mathrm{Y}]=$ Tackon $[\mathrm{X}]$;

for $(\mathrm{i}=0 ; \mathrm{i}<\mathrm{X} ; \mathrm{i}++)$

Newtack[i+1]=Tackon[i];

for $(\mathrm{j}=\mathrm{X}+1 ; \mathrm{j}<$ (Mred-Nred); $\mathrm{j}+)$

Newtack[j]=Tackon[j];

BLOCK_COL_FIND_X(Newtack,g->p[NextToFind],block->p[NextToFind],

\#ifdef DEBUG bred,Ared,Mred,Nred,check);

\#endif

fprintf(check, " $\mathrm{l} n$ it will be \%f $\ln$ ",fabs(g->p[(NextToFind)]

[(int)block->p[NextToFind][Y]]));

$1 *$ Check to ensure the newly created $\mathrm{g}$ vector is independent *I

$1 *$ of previously created ones in branch

*I

if (fabs(g->p[(NextToFind)][(int)block->p[NextToFind][Y]]) >SMALL) if (NextToFind <(Mred-Nred))

\{

\#ifdef DEBUG

fmat_pr(check, "block", block);

fmat_pr(check, "solutions", g);

for $(\mathrm{i}=0$; $\mathrm{i}<$ (Mred-Nred); i+t)

\#endif

fprintf(check, "Ord \%d",Newtack[i]);

$/ *$ if more g vectors are needed it goes to next level $* /$

RestofSoln(Mred, Nred, NextToFind, block, g, bred, Ared,

\} Newtack, Changed, check);

else

SystemComplete $=$ TRUE;

if (!SystemComplete)

block->p[NextToFind] $[Y]=$ Newtack[0];

\#ifdef DEBUG

fprintf(check, "ln $* * * * * * * * * * * * * * * * * * * * * * * * * * * * * * * * * * * \ln )$ ); \#endif

$\underset{3}{\mathrm{X}++}$ \}

free (Newtack);

free (Changed);

\} 


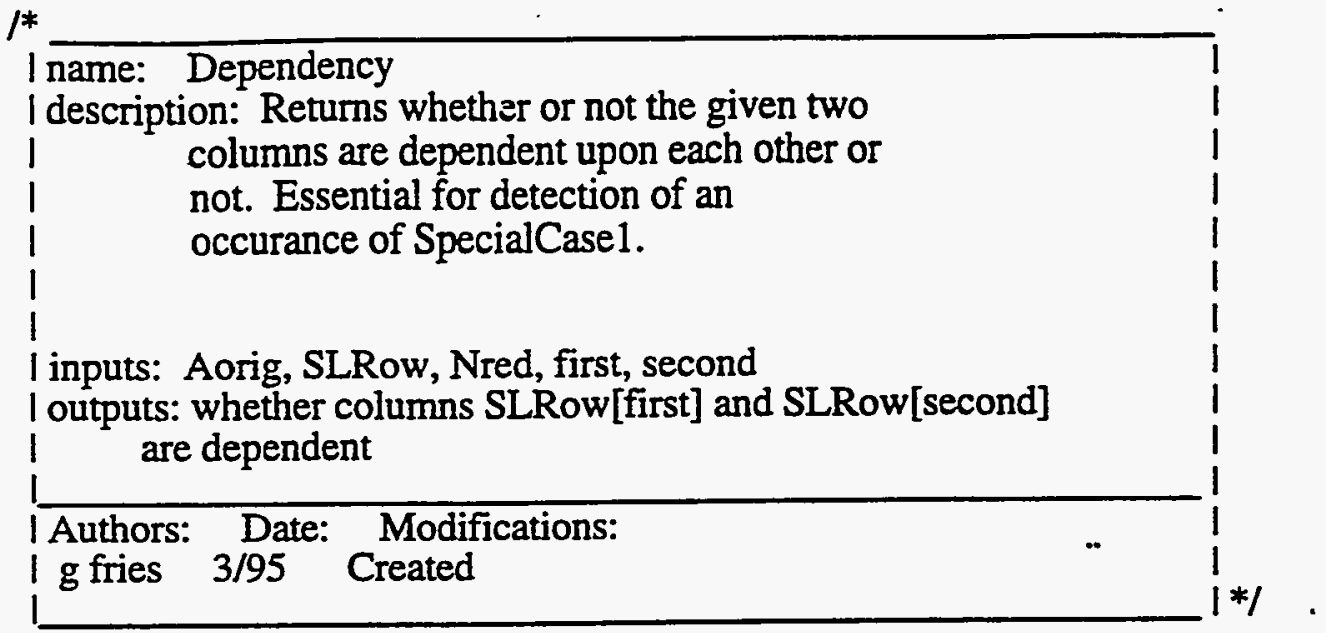

int Dependency (MATRIX *Atemp, int *SLRow, int Nred, int first, int second)

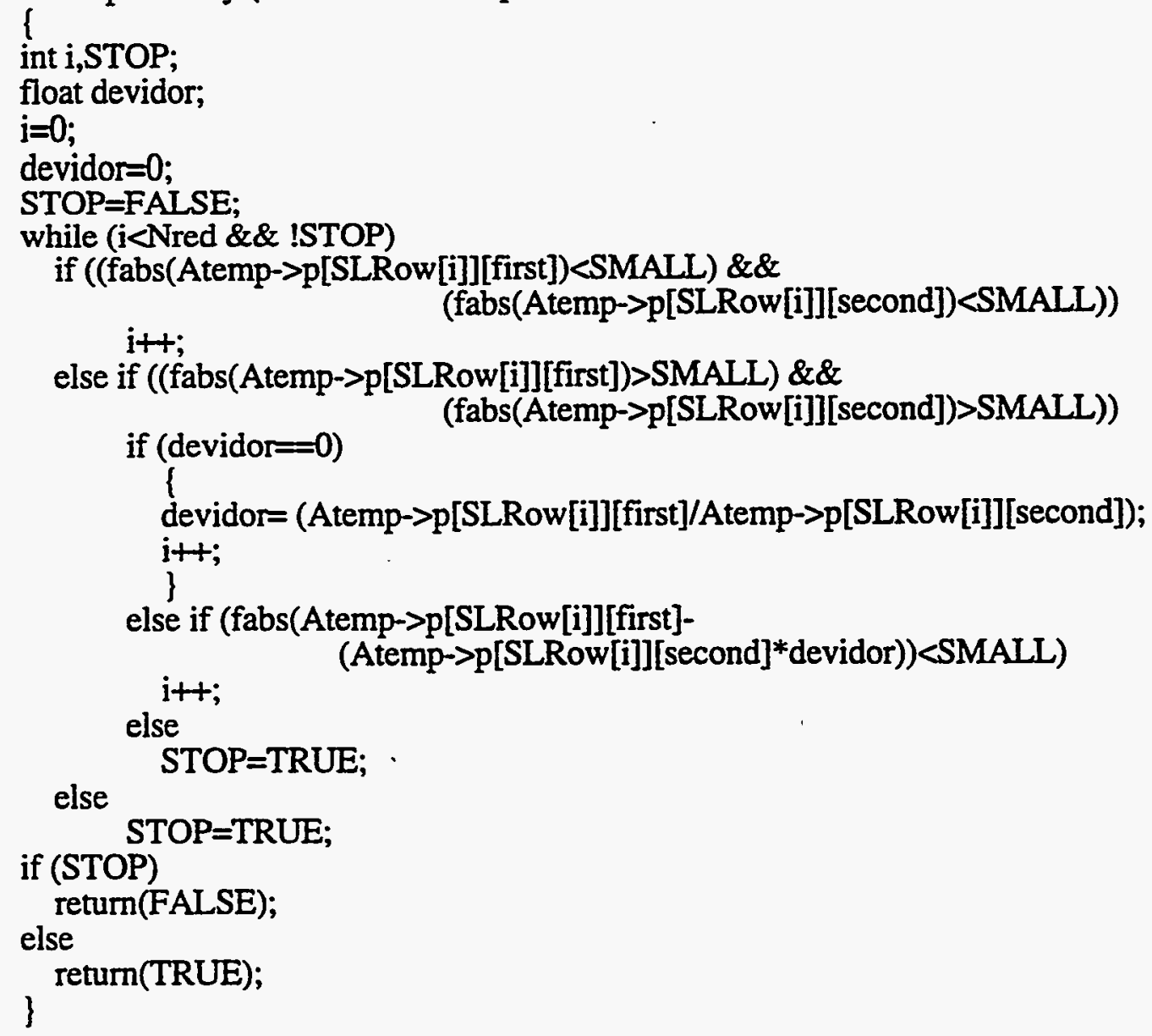




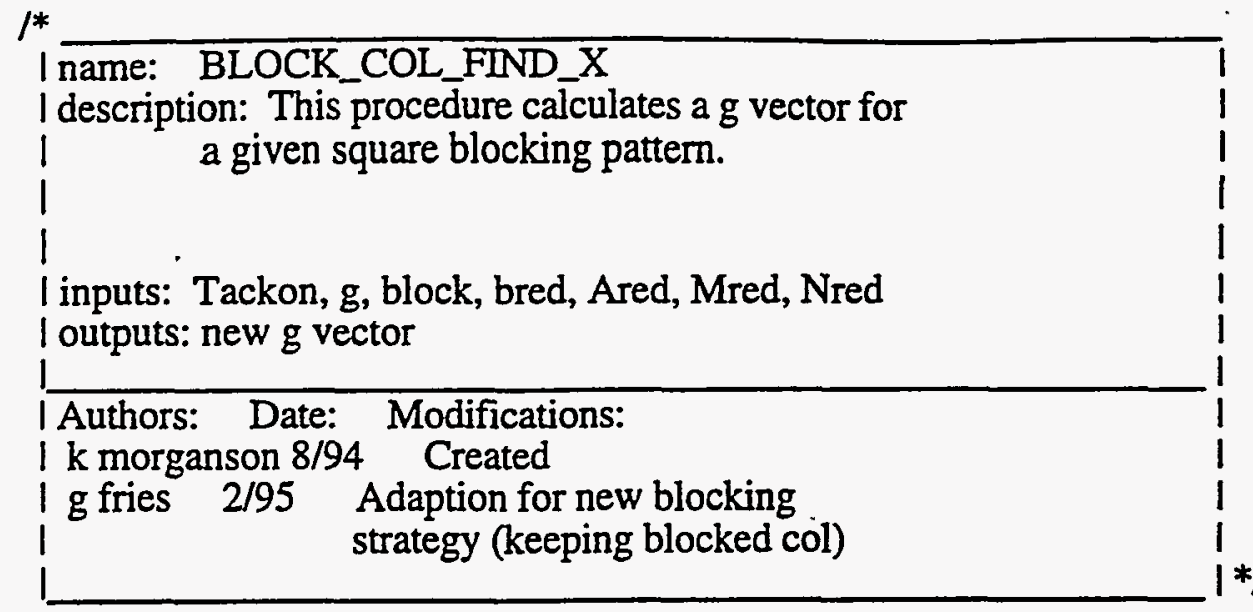

\section{BLOCK_COL_FIND_X (int *Tackon, float *g, float *block, MATRIX *b, MATRIX *A, int Mred, int Nred, FILE *check)}

l

int $r, I, i, j, k$;

float blocktemp[Nred], temp;

MATRIX *Atemp, *gtemp, *btemp;

Atemp = mat_malloc(Nred,Nred);

gtemp= mat_malloc(Nred,1);

btemp = mat_malloc(Nred,1);

for $(i=0 ; i<N r e d ; i++)$

btemp->p[i][0]=b->p[i][0];

$/ *$ the columns blocked need to be listed from smallest to largest */

for $(\mathrm{i}=0 ; \mathrm{i}<($ Nred $) ; \mathrm{i}++)$

blocktemp[i] $=$ (int)block[i];

for $(i=(N r e d-1) ; i>0 ; i--)$

for $(j=i-1 ; j>=0 ; j-)$

if (blocktemp[i]<blocktemp[j])

l

temp=blocktemp[i];

blocktemp[i]=blocktemp[j];

blocktemp[j]=temp;

\}

$\mathrm{k}=0 ; \mathrm{I}=0$;

for $(\mathrm{i}=0$; $\mathrm{i}<$ Mred; $\mathrm{i}++)$

if (blocktemp $[k]=$ i)

i

for $(j=0 ; j<N r e d ; j++)$

Atemp->p[j][k] = A->p[j][i];

\}

$\mathrm{k}++$;

else

$\mathrm{It+}$

\#ifdef DEBUG

fmat_pr(check, "asqr", Atemp); 
\#endif

mat_pseudoinv(Atemp);

gtemp = mat_mul2(Atemp, btemp);

\section{\#ifdef DEBUG}

fprintf(check, "In gvector");

for ( $\mathrm{i}=0$; $\mathrm{i}<\mathrm{Nred}$; ++ )

fprintf(check, "\%f ", gtemp->p[i][0]);

\#endif

/*Mow add a zero to where column was blocked */

$j=0 ;$

for ( $\mathrm{i}=0$; $\mathrm{i}<$ Nred; $\mathrm{i}+\mathrm{H})$

g[(int)blocktemp [i]] =gtemp->p[i][0];

for $(j=0 ; j<($ Mred-Nred $) ; j+)$

g[Tackon $[j]]=0.0 \mathrm{e} 0$;

mat_free(Atemp);

mat_free(gtemp);

mat_free(btemp);

\} 


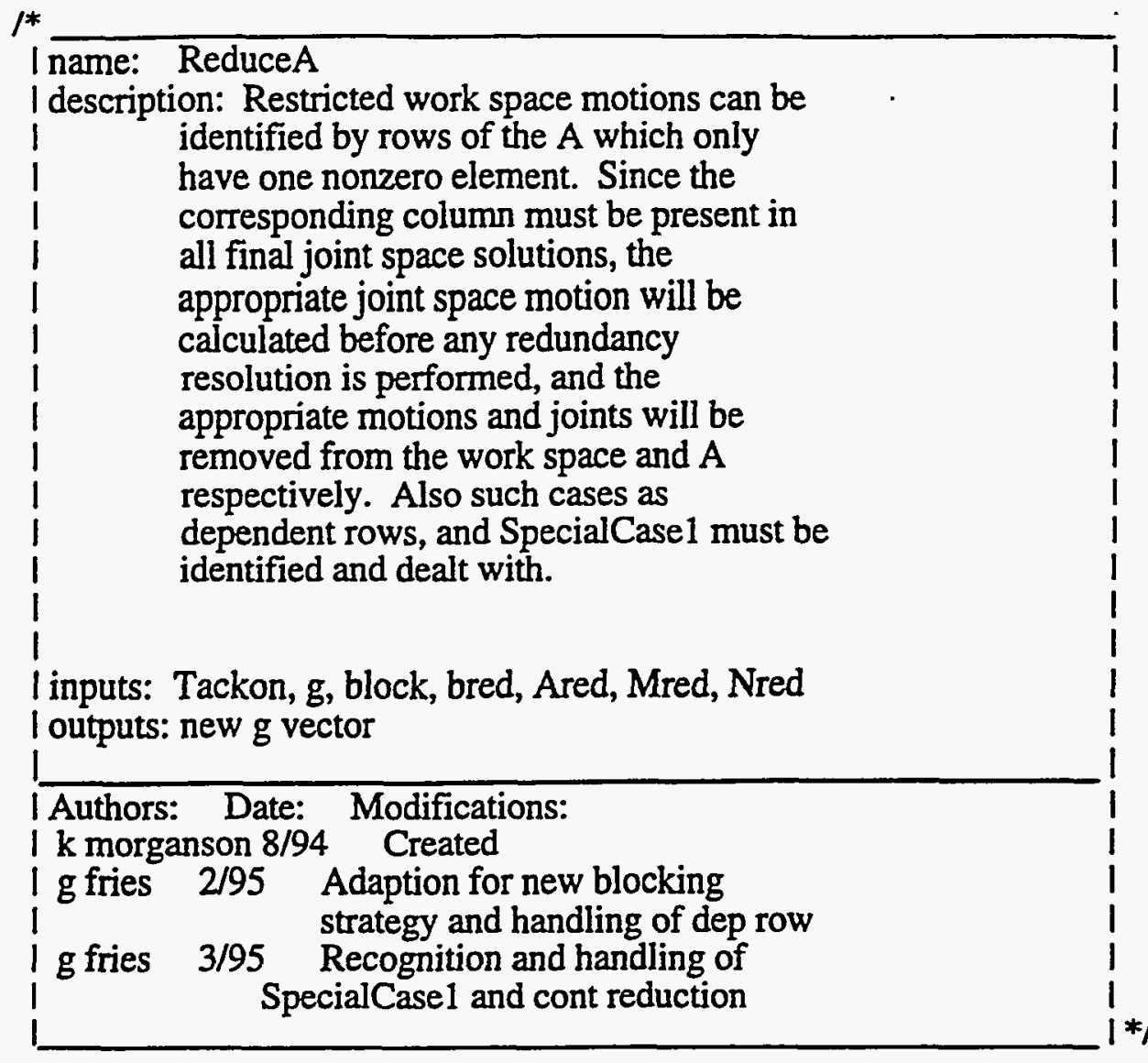

ReduceA(Solutions *FSP_data, MATRIX *Aorig, MATRIX *Ared, MATRIX *borig, MATRIX *bred, MATRIX *Xelim, int* ColElim,int* RowElim, int *NumSpg, MATRIX *Specialg)

int

$\mathrm{i}, \mathrm{j}, \mathrm{k}, \mathrm{m}, \mathrm{r}, \mathrm{p}, \quad / *$ loop counters */

StillChecking, $/ *$ flag to mark when all nonredundancies are gone */

Stop,Stop2, $\quad / *$ Used as loop flag in detection for SpecialCase1\&2 */

ClassANum, /*Number of ClassificationA columns, for SpecialCase2 * *

reference, $\quad / *$ Reference position used for SpecialCase2 row */

LastNred, $\quad 1 *$ Check if all rows have been looked at *l

nullity, $\quad / *$ Used in mat_null when determining depend rows $\quad *$ /

nul_count, $\quad /$ Loop counter for dependent rows */

Restriction, /*num of joints which contrib to a work space d.o.f. */

DoneRed, $\quad$ /*Keeps track if reduction is done, loop has executed */

/*without any reduction */

*SLRow, $\quad / *$ Stands for Still Left Row, stores remaining non $\quad *$ /

/*eliminated rows in sequence */

*SLCol, /*Stands for Still Left Column, stores remaining non */

* $\quad / *$ eliminated columns in sequence $*$ *

*ClassA, $\quad 1 *$ Array used to store ClassificationA columns $\quad * 1$

count,count2, /*Position markers when copying over SLCol when the */

position, $\quad l^{*}$ SpecialCasel has been found */

nonzerocol, $\quad / *$ Number of nonzero col when check for SpecialCase1 */

*Nonzero, /*Stores values of nonzero col when check for SCl 
NonZeroCol, $\quad / *$ Column which has nonzero element for give row

Flag, $1 *$ Used in SC2 for an undefined beta value

Goin; $l^{*}$ "Go in" for entance into ClassA for SC2

double btemp $[N]$; $/ *$ Holds the $b$ vector as it is modified by backsub float

$\mathrm{K} 2$,

/*Matrix condition number: sv_max/sv_min

*Constant,

/*Constant values used when comparing ClassificationA /*columns

beta;

/*Value used for determining SpecialCase2

MATRIX

*n,

$/ *$ null space vectors

*Atrans,

/*Transpose matrix used when looking for depend rows

*AElim; $/ *$ Matrix used to find null vectors when dealing with /* SpecialCase1

I* allocation of memory *

SLRow $=\left(\right.$ int $\left.{ }^{*}\right)$ malloc $((\mathrm{N}) *(\operatorname{sizeof}($ int $)))$;

SLCol $=($ int $*)$ malloc $((M) *($ sizeof $($ int $)))$;

Nonzero $=($ int $*)$ malloc $((M) *($ sizeof $($ int $)))$;

ClassA = (int *) malloc $((M) *($ sizeof $($ int $)))$;

Constant $=($ float $*)$ malloc $((\mathrm{N}) *($ sizeof $($ int $)))$;

$\mathrm{n}=$ mat_malloc(M,M);

/* initialize variables */

FSP_data $\rightarrow$ Nred $=N ; \quad$ * number of rows in the reduced $A *$ /

FSP_data- $>$ Mred $=M ; \quad 1 *$ number of columns in the reduced $A \quad * /$

*NumSpg $=0$;

for $(\mathrm{i}=0 ; \mathrm{i}<\mathrm{N} ; \mathrm{i}++)$

\{

SLRow[i]=i;

RowElim $[i]=0$;

btemp[i]=borig $->\mathrm{p}[\mathrm{i}][0]$;

\}

for $(i=0 ; i<M ; i+t)$

\{

SLCol[i]=i;

Xelim $\rightarrow p[i][0]=0$;

ColElim $[\mathrm{i}]=0$;

\}

DoneRed=FALSE;

/* start reduction */

while (!DoneRed)

\{

DoneRed=TRUE;

StillChecking $=1$;

I* Check each row for the number of nonzero elements. If only one

$I^{*}$ element is nonzero, solve for the joint space motion, and

$/ *$ modify the $b$ vector so that the appropriate row and column

$I^{*}$ can be eliminated from the A. After a row is eliminated

$/ *$ the remaining A must be rechecked for any new restrictions 


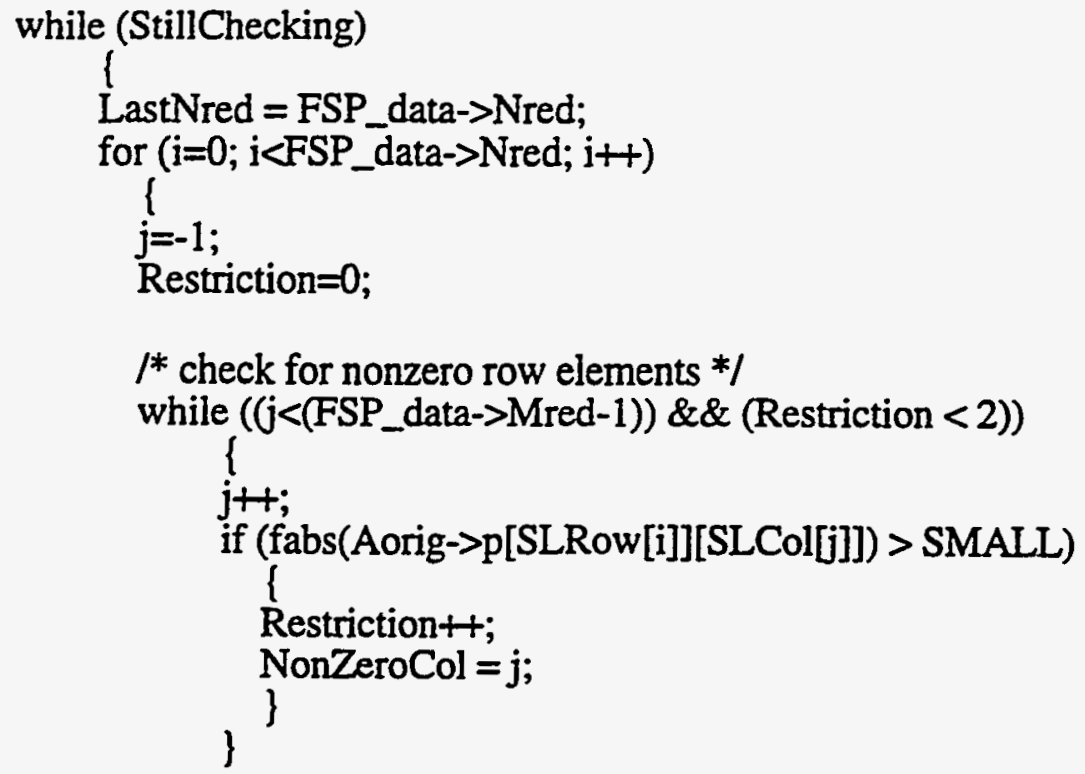

$1 *$ if a row only has one nonzero element, eliminate it from */

I* the A *I

if $(($ Restriction $=1) \& \&$ (RowElim[SLRow[i]] != 1))

I

Xelim->p[SLCol[NonZeroCol] $][0]=$ btemp[SLRow[i]]/Aorig->p[SLRow[i]][SLCol[NonZeroCol]];

for $\left(k=0 ; k<F S P \_d a t a->N r e d ; k++\right)$ btemp[SLRow[k]] = btemp[SLRow[k]]-Aorig->p[SLRow[k]][SLCol [NonZeroCol]]*Xelim->p[SLCol[NonZeroCol]][0];

ColElim[SLCol[NonZeroCol]] $=1$;

RowElim[SLRow[i]]=1;

for $(j=$ NonZeroCol; $j<$ (FSP_data->Mred-1); $j++$ )

SLCol[j]=SLCol[j+1];

for $(j=i ; j<($ FSP_data- $>$ Nred-1); $j++)$

SLRow $[j]=S L R o w[j+1]$;

FSP_data->Nred--;

FSP_data->Mred-;

else $\quad I^{*}$ row of all zeros, also eliminated *

if ((Restriction $==0) \& \&$ (RowElim[SLRow[i]] != 1))

l

RowElim[SLRow[i]]=1;

for $\left(j=i ; j<\left(F S P \_d a t a->N r e d-1\right) ; j+4\right)$

SLRow[j] =SLRow $[j+1]$;

FSP_data->Nred--;

\}

\}

if (FSP_data->Nred $=$ LastNred)

StillChecking $=0$; 


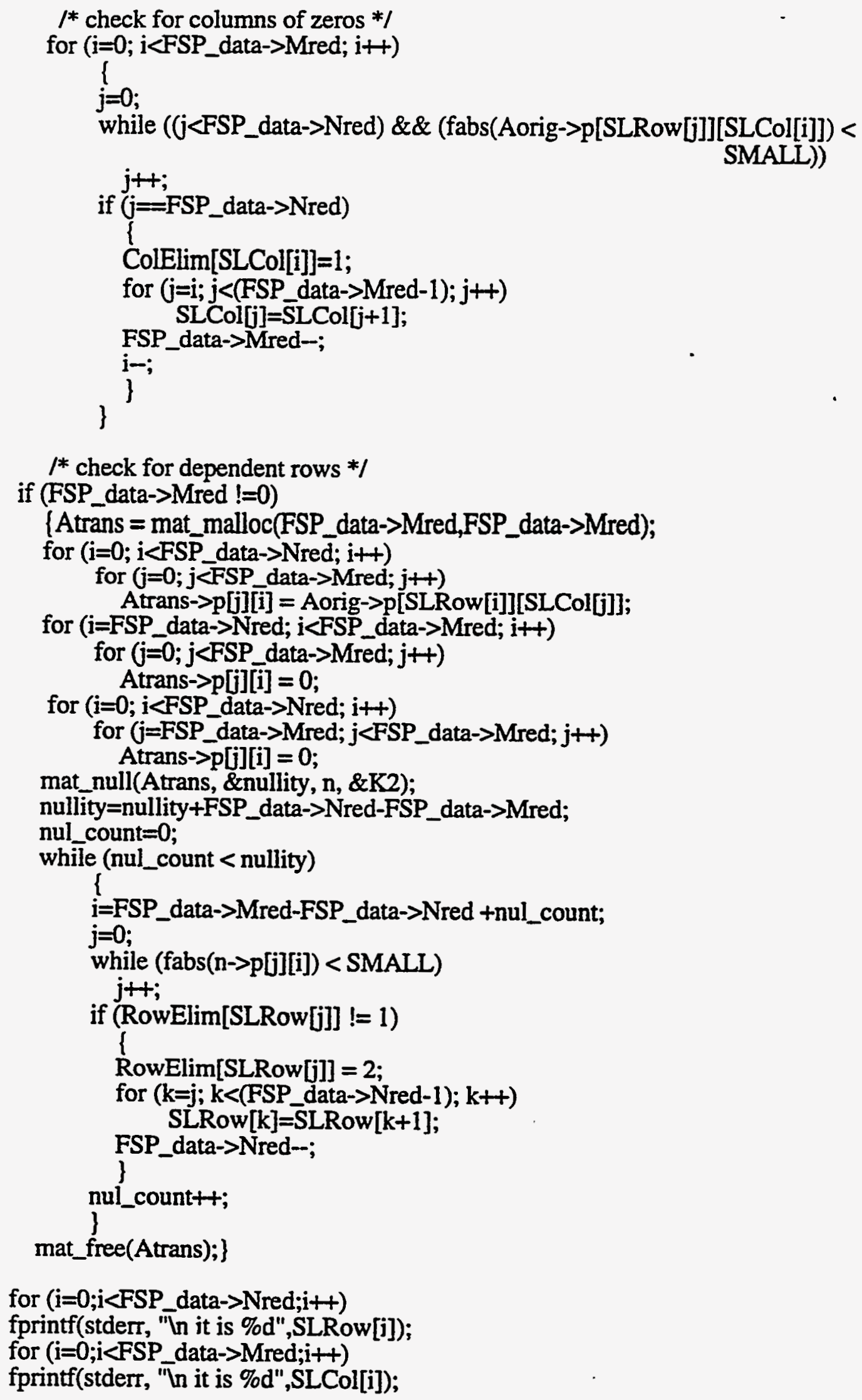

for ( $\mathrm{i}=0$; $\mathrm{i}<F S P$ data- $>$ Nred; $\mathrm{i}++$ )

fprintf(stder, "In it is \%d",SLRow[i]);

for ( $\mathrm{i}=0$;i<FSP_data- $>$ Mred; $\mathrm{i}++$ )

fprintf(stderr, "ln it is \%d",SLCol[i]); 


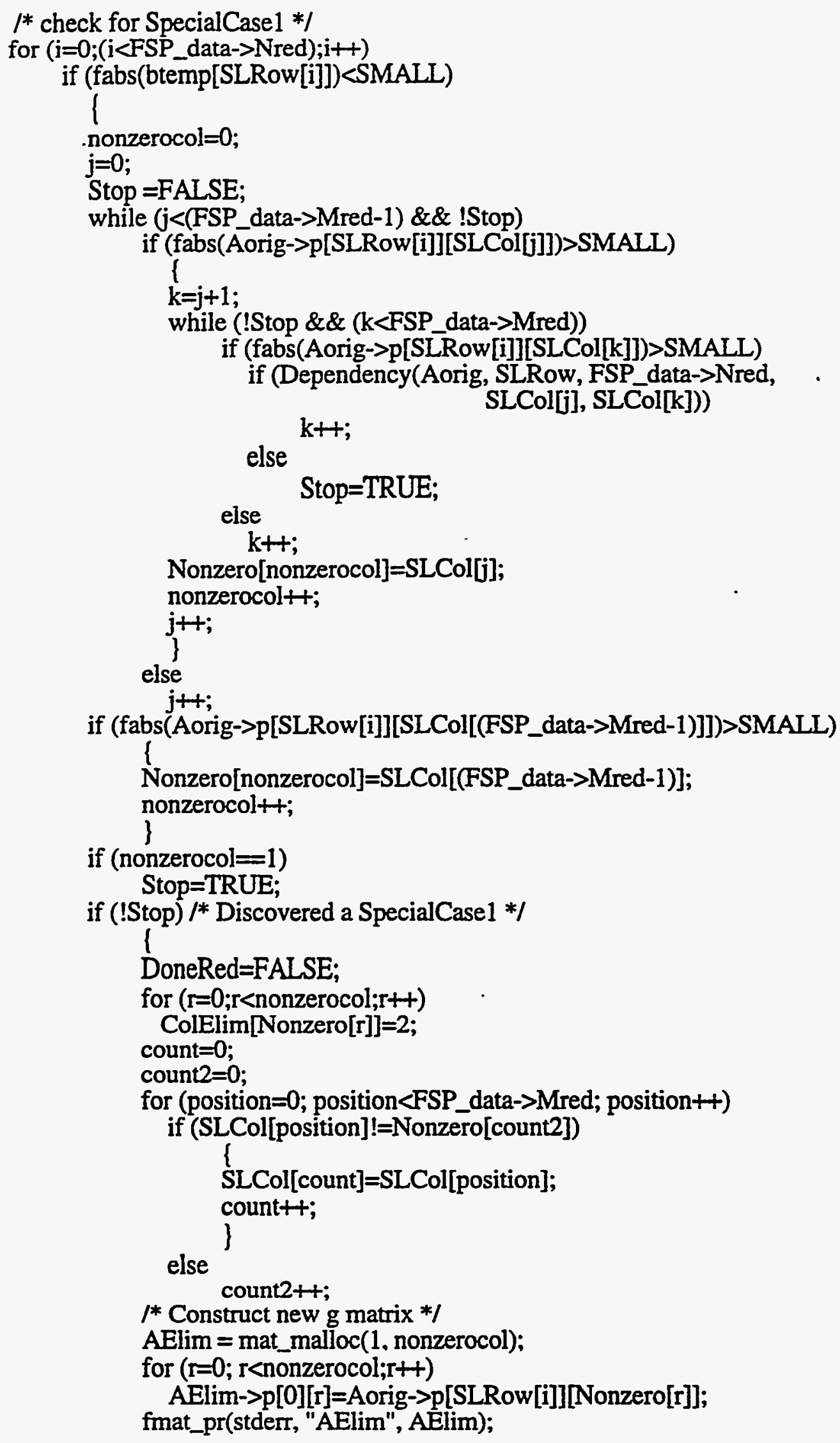


mat_null(AElim, \&nullity, n, \&K2);

fmat_pr(stderr, " $n$ ", n);

mat_free(AElim);

FSP_data- $>$ Mred=FSP_data- $>$ Mred-nonzerocol;

RowElim[SLRow[i]] $=\overline{1}$;

for ( $\mathrm{r}=\mathrm{i} ; \mathrm{r}<($ FSP_data->Nred-1); $\mathrm{r}++)$

SLRow[r]=SLRow[r+1];

FSP_data- $\rightarrow$ Nred--;

for $(m=0 ; m<$ (nonzerocol-1); $m+4)$

\{

for $(r=0 ; r<M ; r+H)$

Specialg $\rightarrow$ p [*NumSpg] $[\mathrm{r}]=0$;

for $(r=0 ; r<$ nonzerocol; $r++)$

Specialg $\rightarrow$ p [*NumSpg][Nonzero $[r]]=n->p[r][m]$;

*NumSpg=*NumSpg+1;

\}

)

\}

/*Check for SpecialCase2*I

$\mathrm{i}=0$;

Stop2=FAISE;

while ((i<(FSP_data->Nred-1)) \&\& (!Stop2))

!

$\mathrm{j}=\mathrm{i}+1$;

while ((j<FSP_data->Nred) \&\& (!Stop2))

I

Stop=FALSE;

Flag=FALSE;

fprintf(stderr, "In Comparing \%d and \%d In",SLRow[i],SLRow[j]);

if (fabs(btemp[SLRow[i]])>SMALL)

else

beta $=$ btemp[SLRow[j]]/btemp[SLRow[i]];

Flag=TRUE;

Class ANum=0;

$\mathrm{k}=0$;

reference=i;

while ((!Stop) \&\& (k<FSP_data->Mred))

\{

Goin=FALSE;

if (fabs(Aorig->p[SLRow[i]][SLCol[k]])<SMALL)

if (!Flag)

else;

Goin=TRUE;

else

!

if (fabs((Aorig->p[SLRow[j]][SLCol[k]]/

Aorig $\rightarrow$ p[SLRow[i]][SLCol[k]])-beta)>SMALL)

Goin=TRUE;

if (Flag)

\}

Goin=TRUE; 


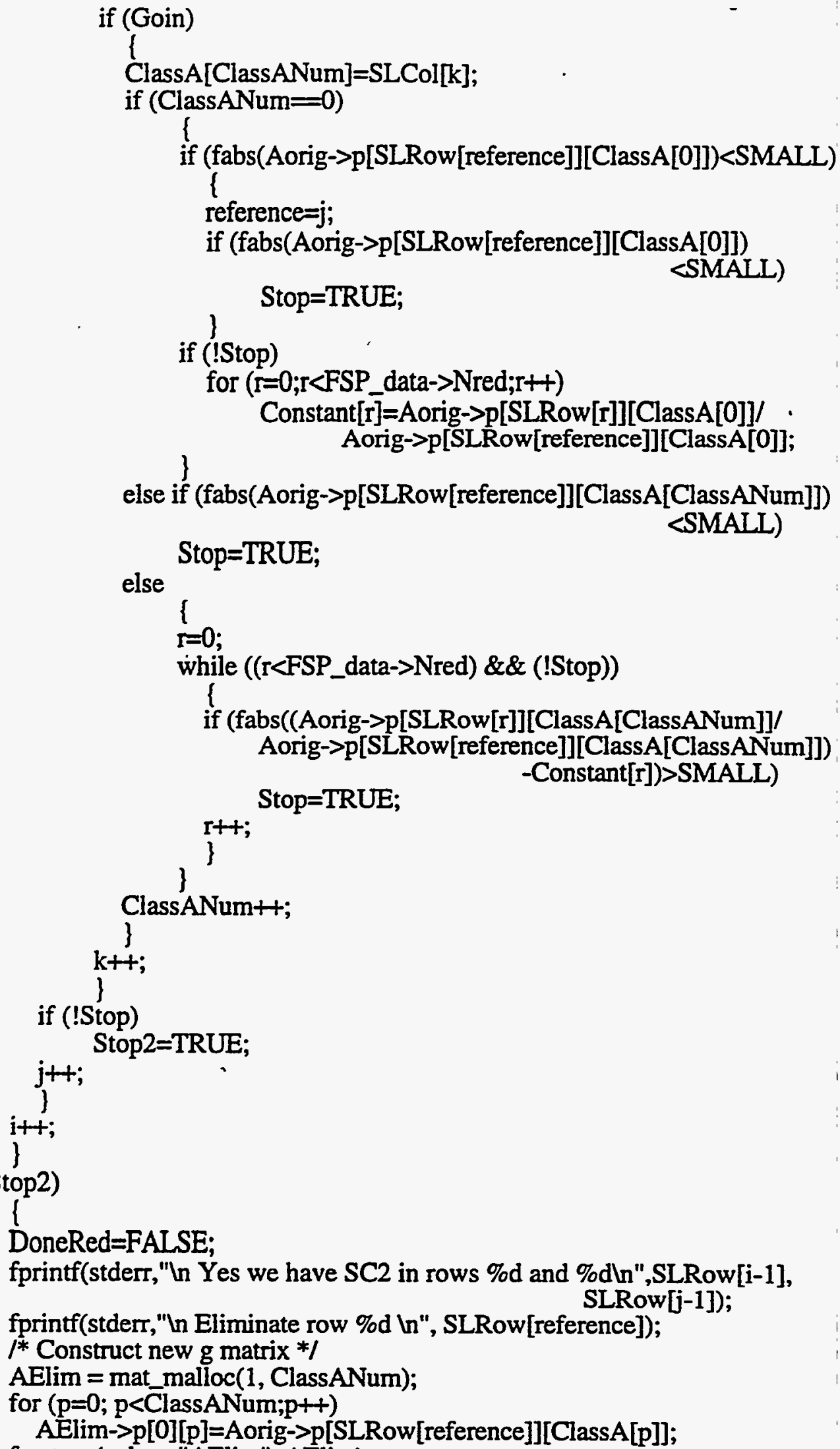




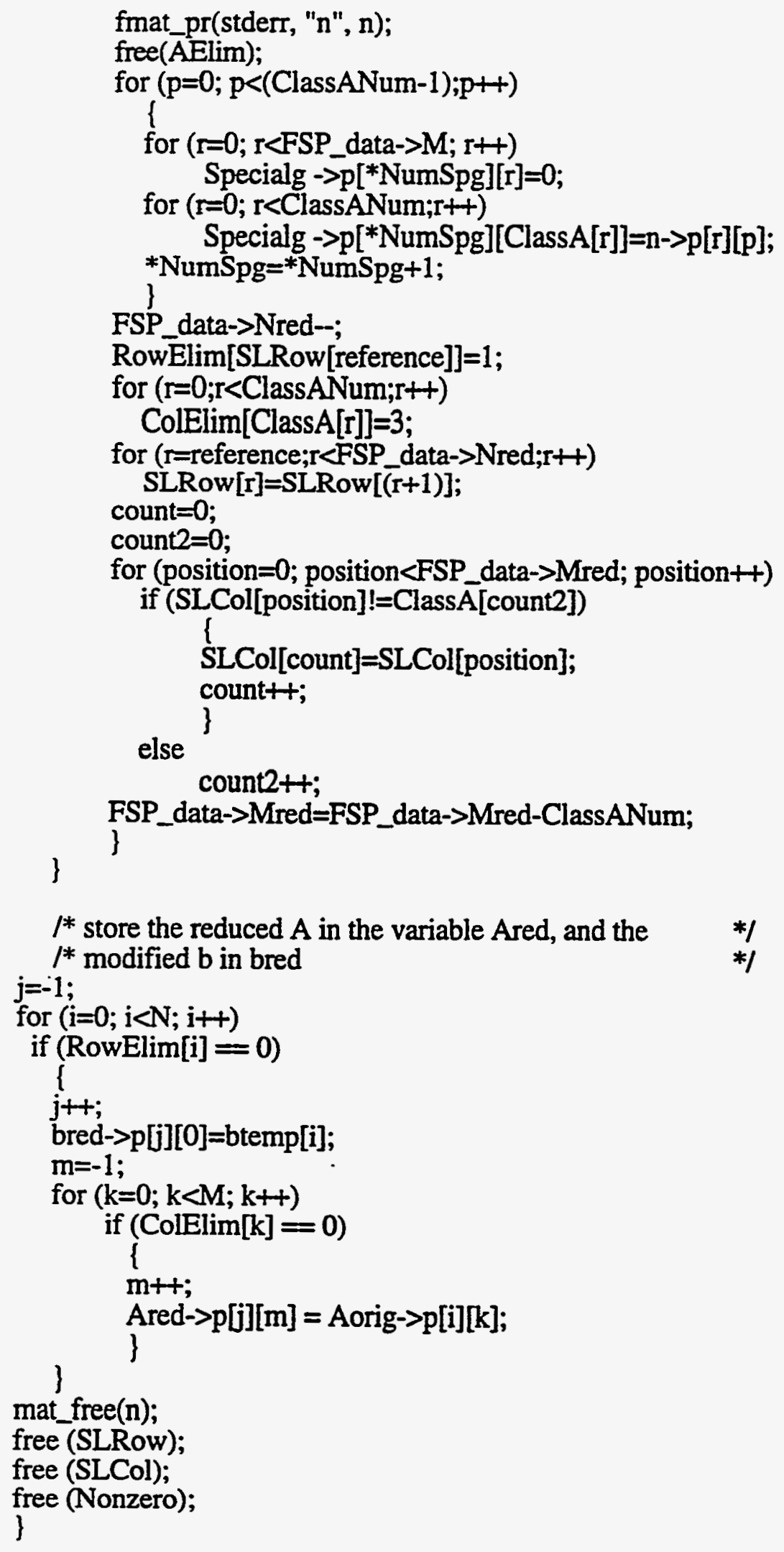




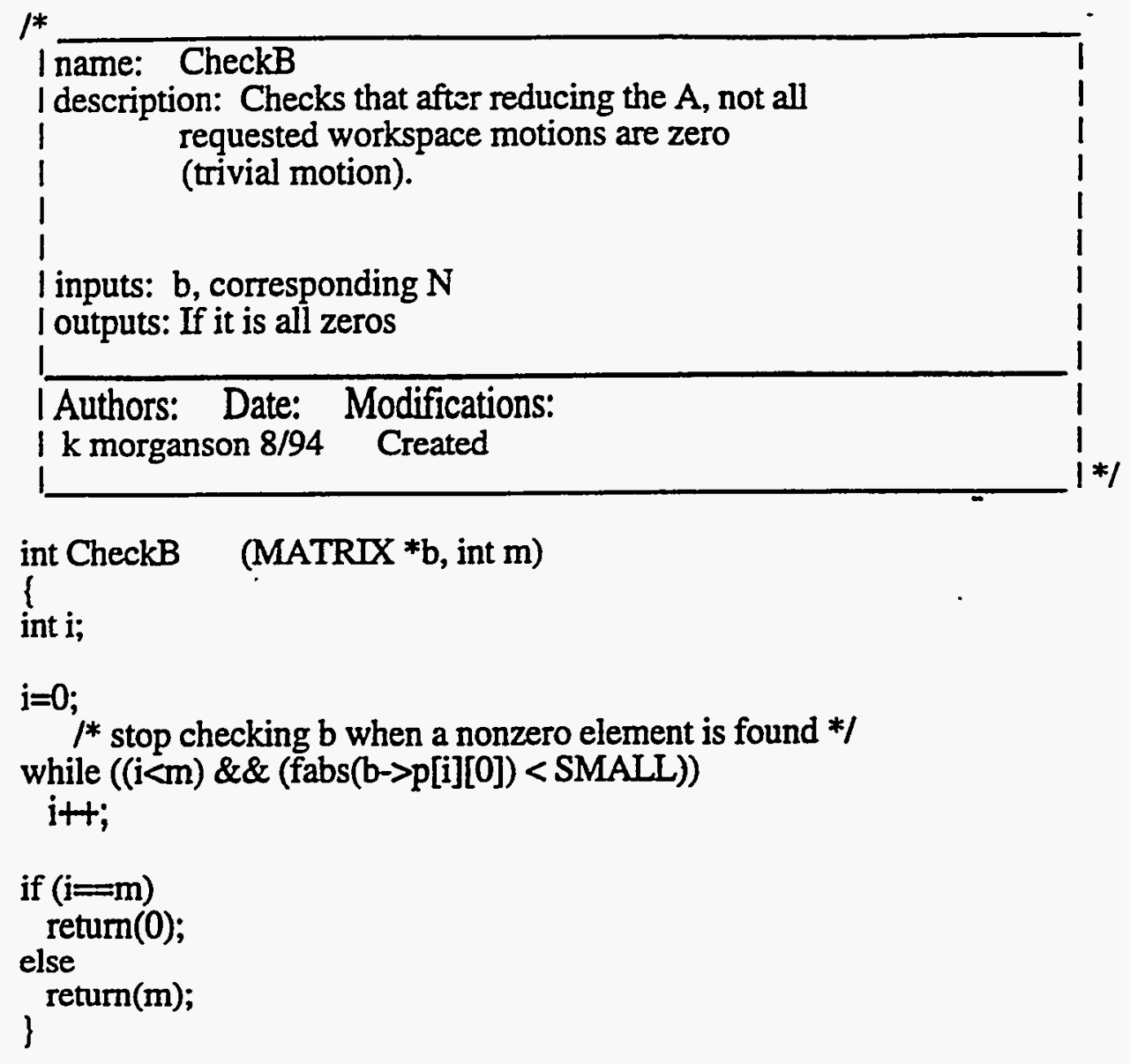

int CheckB (MATRIX *b, int m)

I

int $i$;

$\mathrm{i}=0$;

* stop checking $b$ when a nonzero element is found */

while $((\mathrm{i}<\mathrm{m}) \& \&(\mathrm{fabs}(\mathrm{b} \rightarrow \mathrm{p}[\mathrm{i}][0])<$ SMALL $))$

it+;

if $(i=m)$

return(0);

else

\}

return(m); 


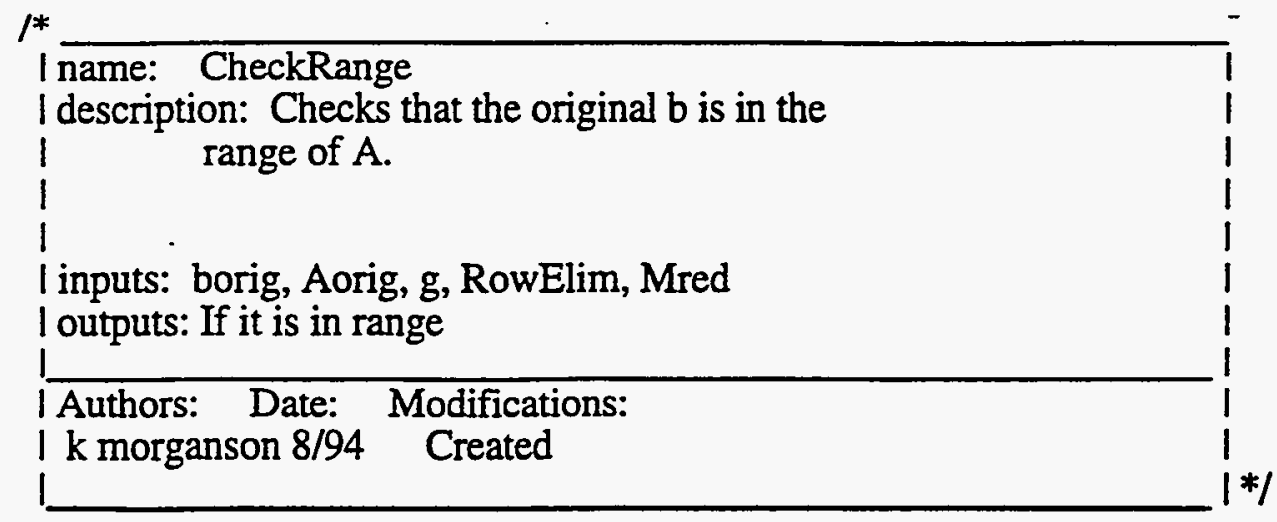

int CheckRange (MATRIX * b, MATRIX * Aorig, MATRIX * g, int *RowElim, int Mred)

\{

int $\mathrm{i}, \mathrm{j}, \mathrm{k}$;

double CheckValue, CheckTemp, CheckTemp2;

for $(\mathrm{i}=0 ; \mathrm{i}<$ (Aorig $\rightarrow$ rows); $\mathrm{i}++$ )

\{

if (RowElim[i] $=2$ )

l

for $(j=0 ; j<(g->$ rows $) ; j++)$

\{

CheckValue $=0.0$;

for $(k=0 ; k<$ (Aorig $\rightarrow>$ cols); $k++)$

CheckValue $+=$ Aorig $\rightarrow p[i][k] *$ g $\rightarrow$ p[j][k];

CheckTemp $=$ fabs (b->p[i][0] - CheckValue);

CheckTemp2 = .01;

if (CheckTemp > CheckTemp2)

\} return (0);

\}

return (1);

\} 


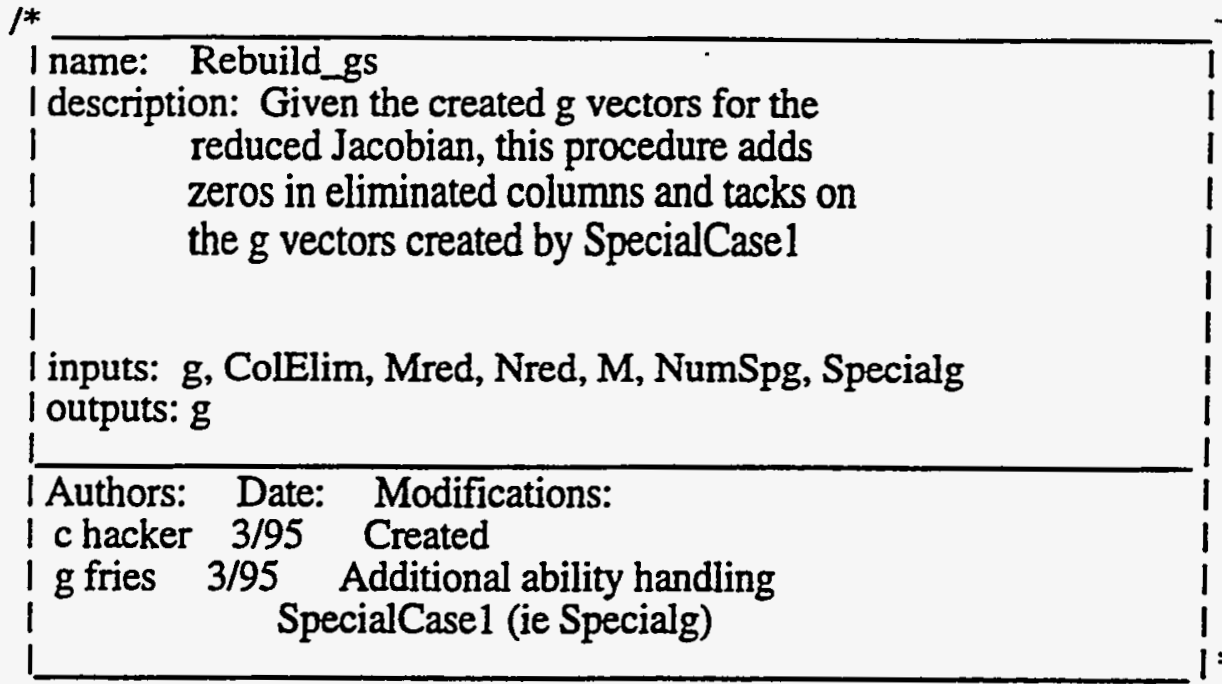

void Rebuild_gs (Solutions *FSP_data, int *ColElim, MATRIX *Specialg,int NumSpg)

( int $\mathrm{i}, \mathrm{j}, \mathrm{k}$;

MATRLX *gtemp;

gtemp $=$ mat_cp2(FSP_data- $>$ g);

$\mathrm{k}=0$;

for $\left(j=0 ; j<F S P \_d a t a->M ; j++\right)$

l

if (ColElim[j] !=0)

l

for (i=0;i<FSP_data->g->rows;i+t)

)

FSP_data- $>\mathrm{g}->\mathrm{p}[\mathrm{i}][\mathrm{j}]=\mathrm{ZERO}$;

else

I

for ( $i=0$;i<FSP_data->g->rows;i+t)

FSP_data- $>\mathrm{g}->\mathrm{p}[\mathrm{i}] \mathrm{j}] \mathrm{j}]=\mathrm{gtemp}->\mathrm{p}[\mathrm{i}][\mathrm{k}]$;

\} $\mathrm{k}++$;

/*Addition of null vectors created by SpecialCase $1 * /$

for (i=(FSP_data->Mred-FSP_data- $>$ Nred +1$) ;$; $<$

for $\left(j=0 ; j<F S P \_d a t a->M ; j++\right)$

(FSP_data- $\rightarrow$ Mred-FSP_data- $>$ Nred $+1+$ NumSpg);i+t)

if (ColElim[j] !=0)

FSP_data- $>\mathrm{g} \rightarrow>\mathrm{p}[\mathrm{i}][\mathrm{j}]=$

else

Specialg->p[(i-FSP_data $>$ Mred+FSP_data- $>$ Nred-1)][j];

FSP_data->g->p[i][j] $=F S P \_d a t a->g->p[0][j]$;

mat_free(gtemp);

\} 


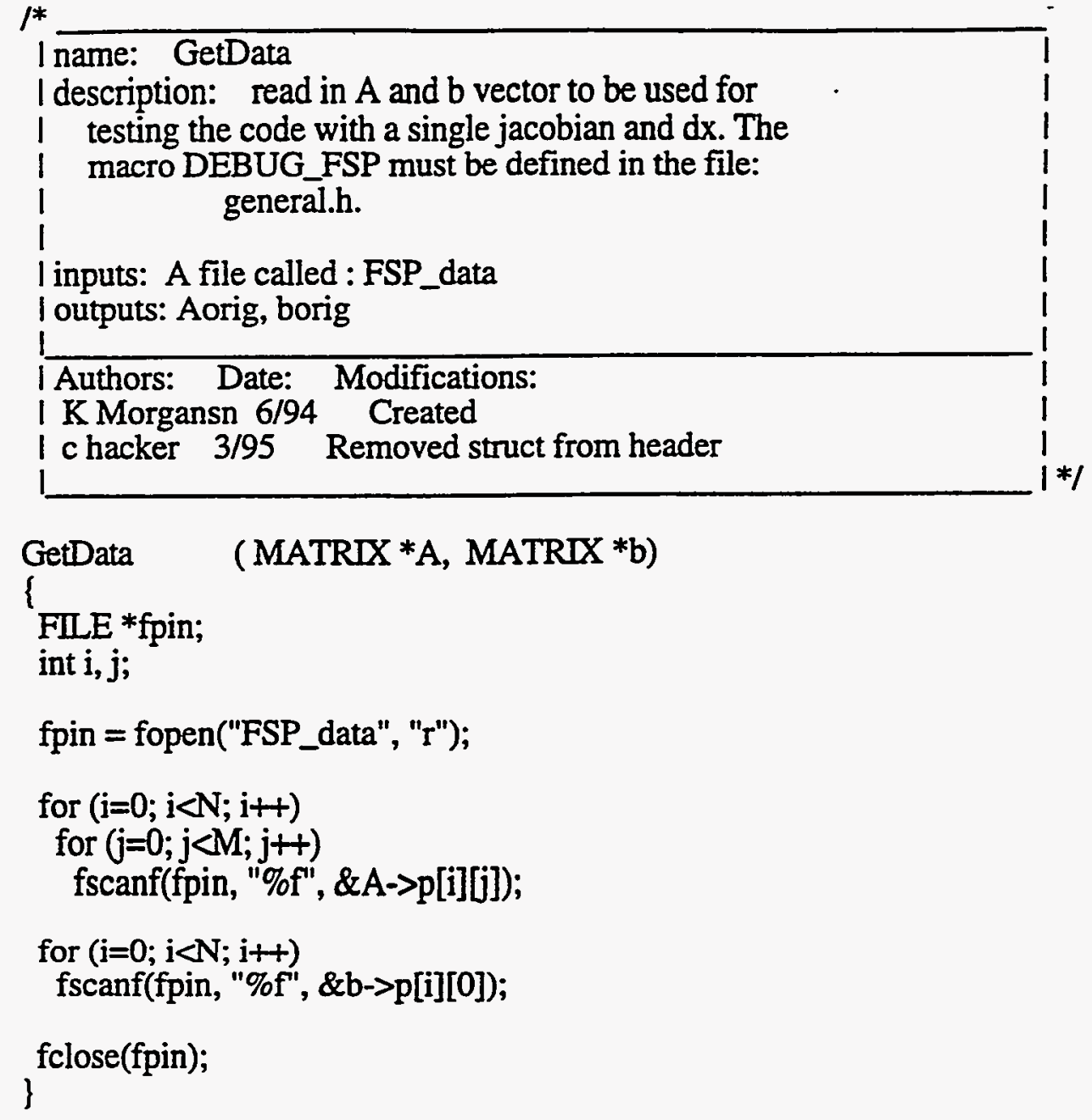




\section{APPENDIX C TRAJECTORIES}

The following trajectories are the five randomly generated trajectories used for timing. Each trajectory started with the manipulator in its "home" position and moved to a specified point. This home position corresponded to an end effector position and initial angles of the manipulator arm, shown below:

Initial end effector $\mathbf{b}:(-0.880,0.254,0.935)$

Hermies initial configuration:

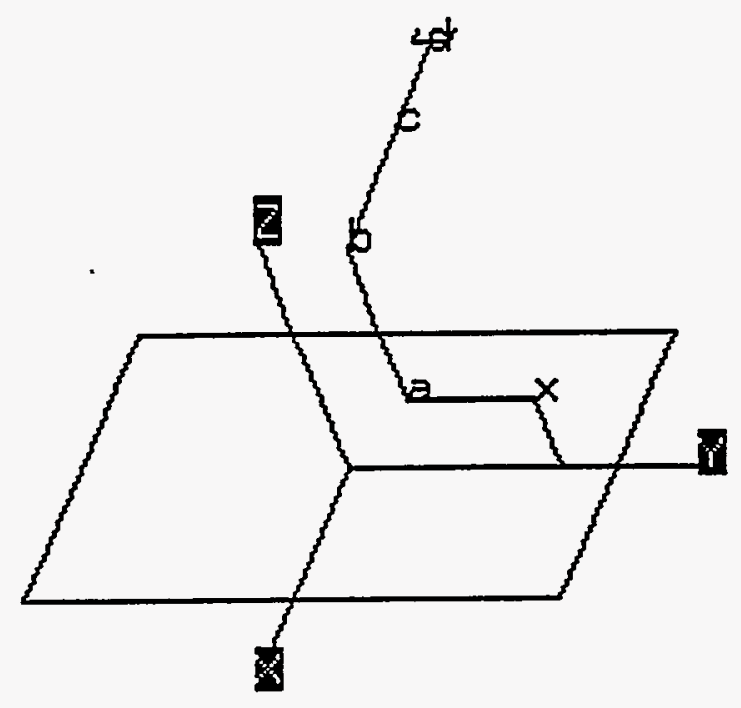

x: $(0.00,180.00,0.00,0.00,0.00,90.00,0.00,0.00,0.00,0.00)$

Airarm initial configuration:

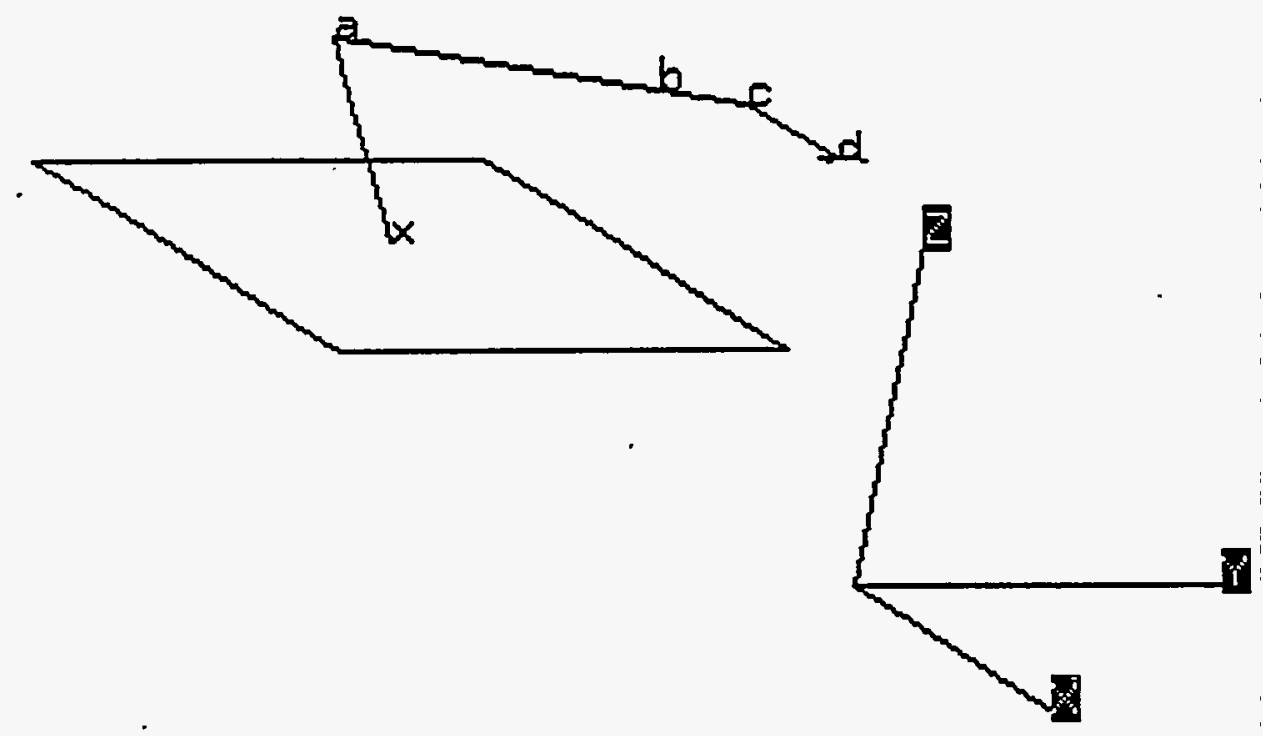

x: $(-45.00,119.96,12.00,30.00,0.00,-153.70,14.55)$ 
As noted before these trajectories were used for both the Hermies and Airarm configuration, and therefore began with the same end effector position. For ease the following examples all present the Hermies system in the start and finish position for each of the trajectories. In addition each trajectory had a corresponding end position for the end effector, which was reached by a straight line. This line was split into discrete steps, and angle displacements were solved for accordingly. for a listing of the new $\mathbf{b}$ for each time step see Âppendix D.

\section{C.1 Trajectory 1}

Starting Position:

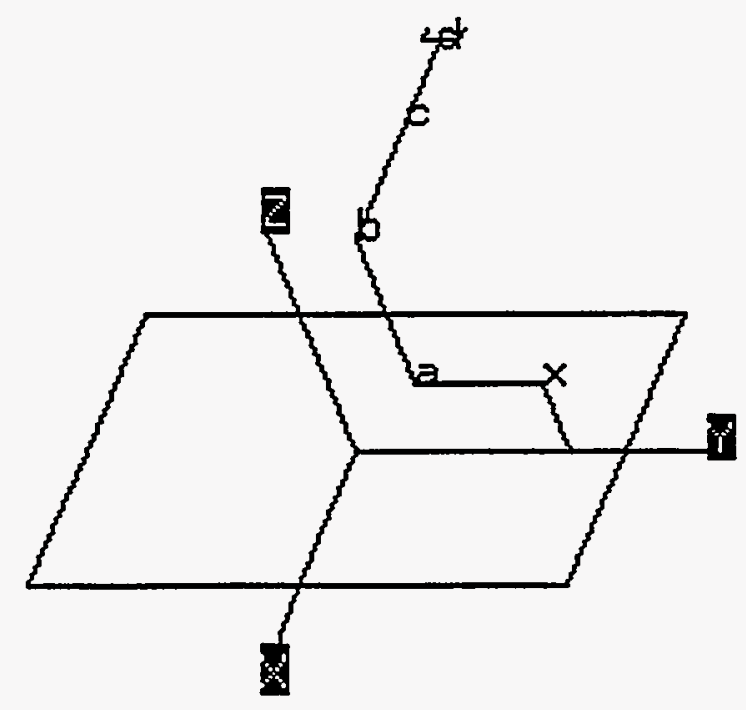

Finish Position:

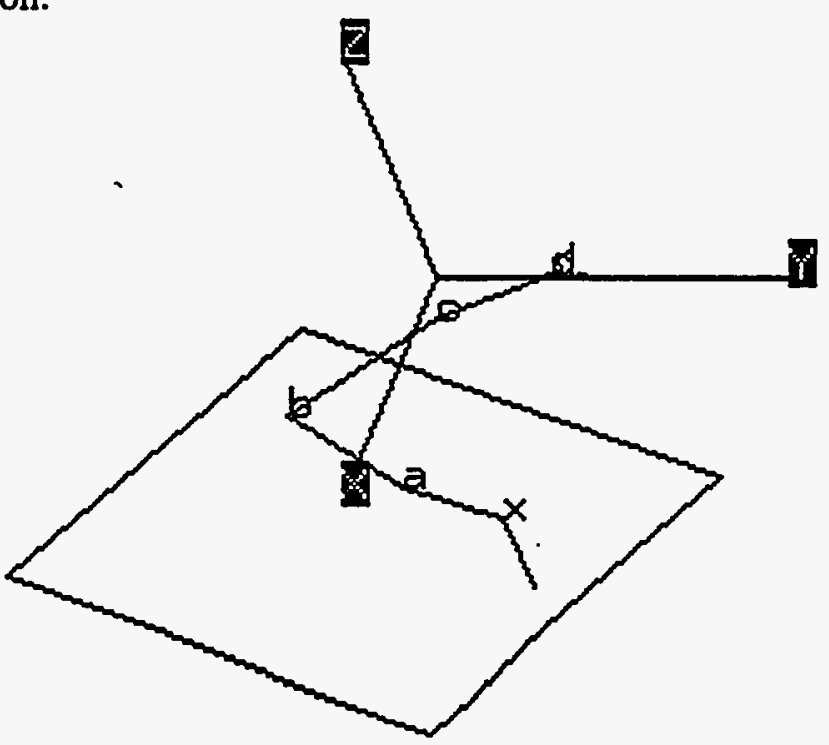

Trajectory 1 consisted of 178 time steps. 


\section{C.2 Trajectory 2}

Starting Position:

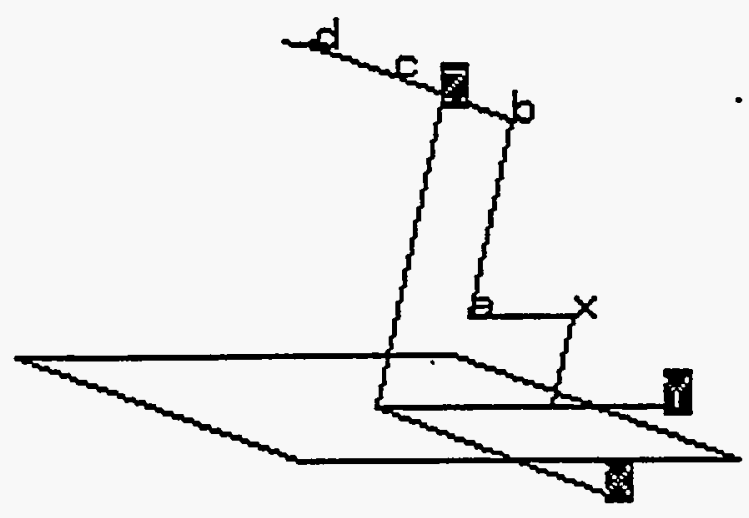

Finish Position:

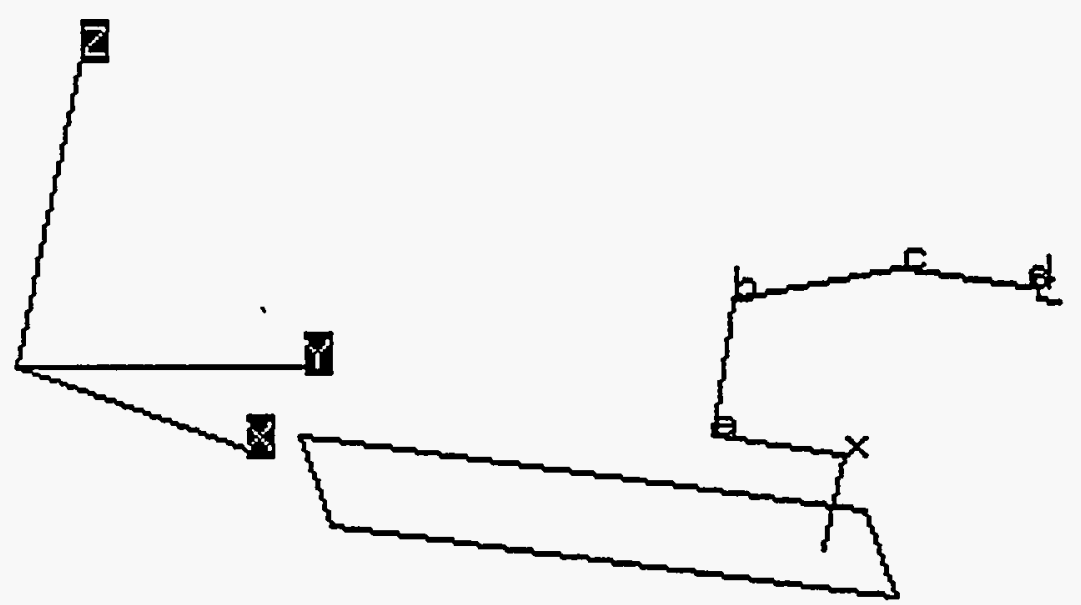

Trajectory 2 consisted of 326 time steps. 


\section{C.3 Trajectory 3}

Starting Position:

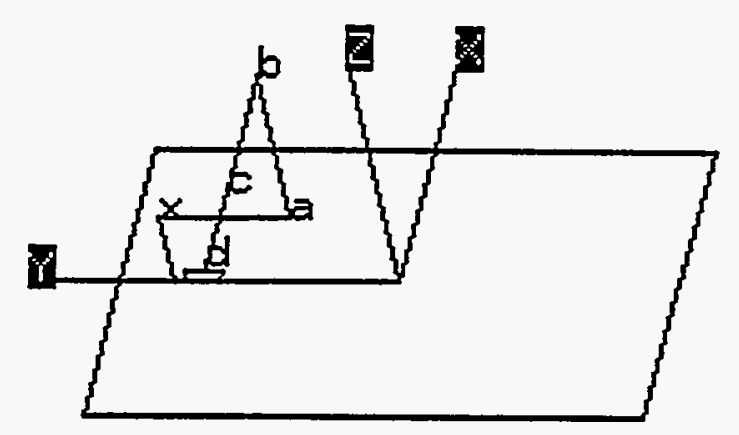

Finish Position:

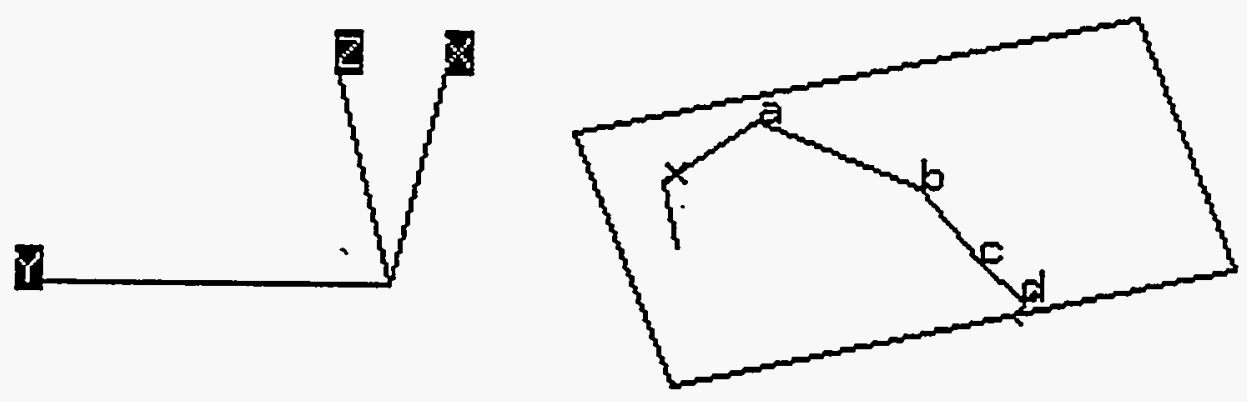

Trajectory 3 consisted of 184 time steps. 


\section{C.4 Trajectory 4}

Starting Position:

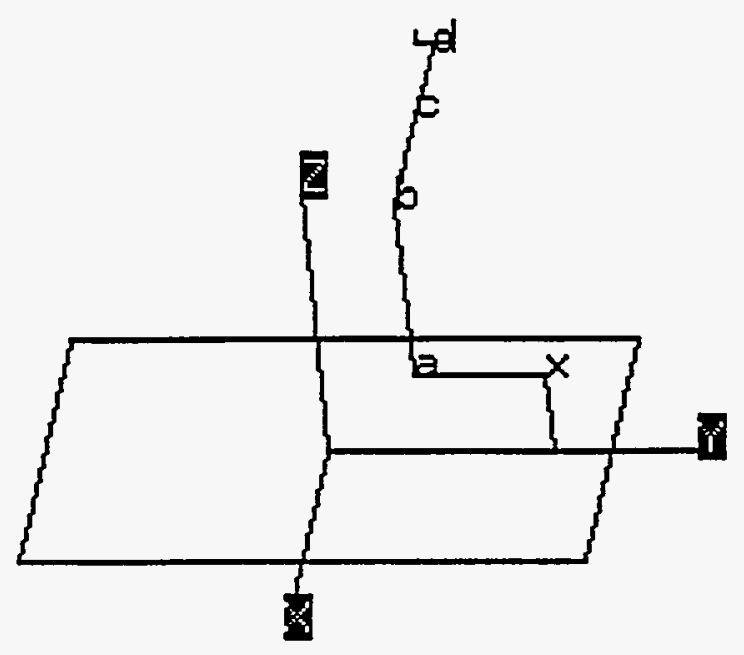

Finish Position:

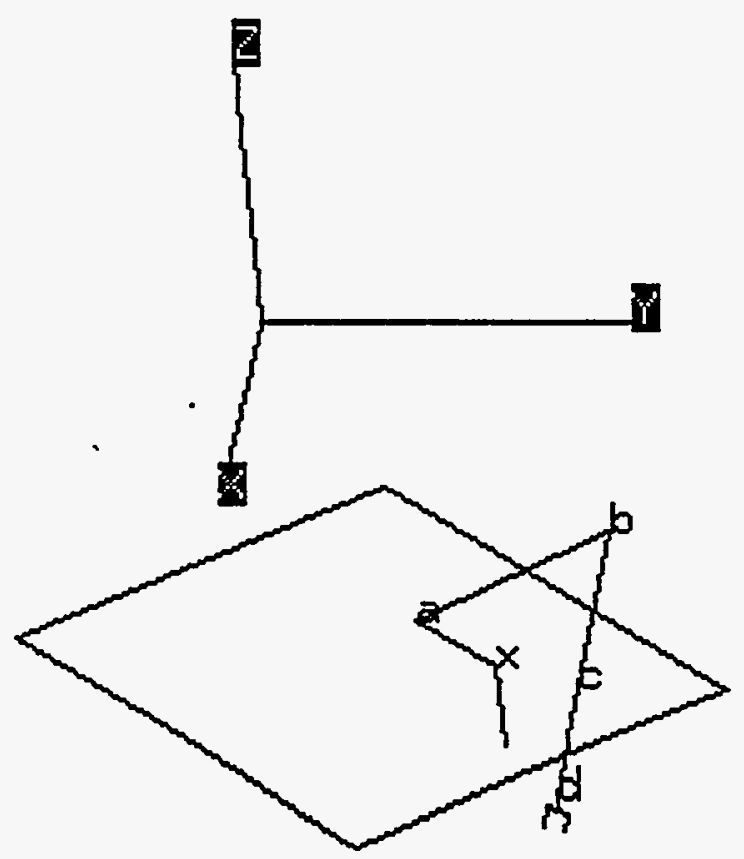

Trajectory 4 consisted of 298 time steps. 


\section{C.5 Trajectory 5}

Starting Position:

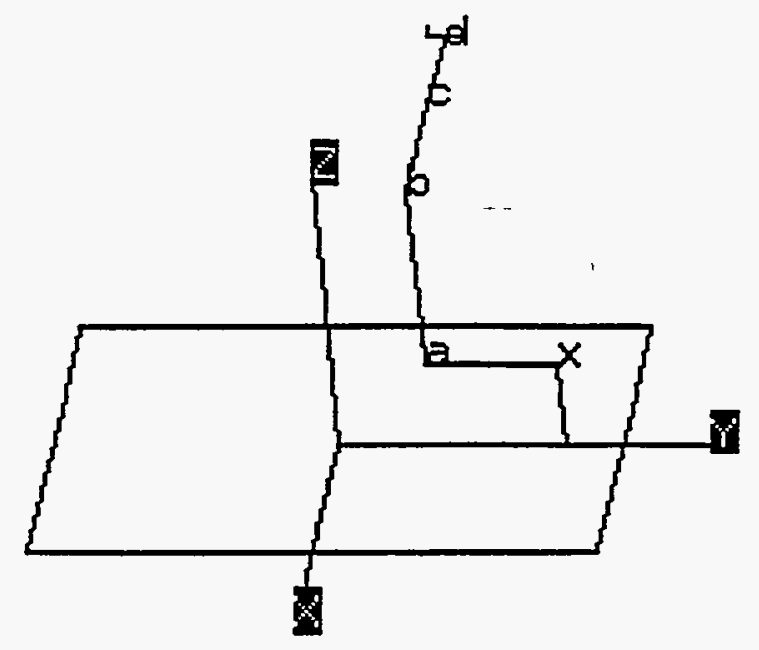

Finish Position:

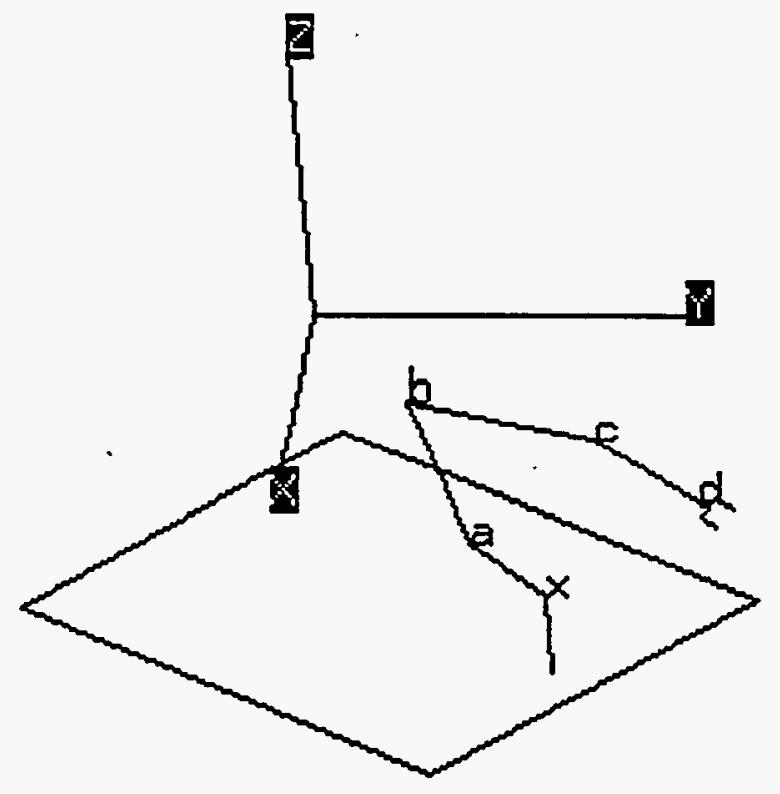

Trajectory 5 consisted of 271 time steps. 
D.1 Trajectory 1

\section{APPENDIX D \\ TRAJECTORY DATA}

180

$\begin{array}{lll}-0.880000 & 0.254000 & 0.935000 \\ -0.869968 & 0.257386 & 0.935000 \\ -0.859936 & 0.260772 & 0.935000 \\ -0.849904 & 0.264158 & 0.935000 \\ -0.839872 & 0.267544 & 0.935000 \\ -0.829840 & 0.270930 & 0.935000 \\ -0.819807 & 0.274316 & 0.935000 \\ -0.809775 & 0.277702 & 0.935000 \\ -0.799743 & 0.281088 & 0.935000 \\ -0.789711 & 0.284474 & 0.935000 \\ -0.779679 & 0.287860 & 0.935000 \\ -0.769647 & 0.291246 & 0.935000 \\ -0.759615 & 0.294632 & 0.935000 \\ -0.749583 & 0.298018 & 0.935000 \\ -0.739551 & 0.301404 & 0.935000 \\ -0.729519 & 0.304790 & 0.935000 \\ -0.719487 & 0.308176 & 0.935000 \\ -0.709455 & 0.311563 & 0.935000 \\ -0.699422 & 0.314949 & 0.935000 \\ -0.689390 & 0.318335 & 0.935000 \\ -0.679358 & 0.321721 & 0.935000 \\ -0.669326 & 0.325107 & 0.935000 \\ -0.659294 & 0.328493 & 0.935000 \\ -0.649262 & 0.331879 & 0.935000 \\ -0.639230 & 0.335265 & 0.935000 \\ -0.629198 & 0.338651 & 0.935000 \\ -0.619166 & 0.342037 & 0.935000 \\ -0.609134 & 0.345423 & 0.935000 \\ -0.599102 & 0.348809 & 0.935000 \\ -0.589069 & 0.352195 & 0.935000 \\ -0.579037 & 0.355581 & 0.935000 \\ -0.569005 & 0.358967 & 0.935000 \\ -0.558973 & 0.362353 & 0.935000 \\ -0.548941 & 0.365739 & 0.935000 \\ -0.538909 & 0.369125 & 0.935000 \\ -0.528877 & 0.372511 & 0.935000 \\ -0.518845 & 0.375897 & 0.935000 \\ -0.508813 & 0.379283 & 0.935000 \\ -0.498781 & 0.382669 & 0.935000\end{array}$

$\begin{array}{ll}-0.488749 & 0.386055\end{array}$

$-0.478717$

0.389441

$-0.468684$

0.392827

$-0.458652$

$-0.448620$

$-0.438588$

$-0.428556$

$-0.418524$

$-0.408492$

$-0.398460$

$-0.388428$

$-0.378396$

$-0.368364$

$-0.358331$

$-0.348299$

$-0.338267$

$-0.328235$

$-0.318203$

$-0.308171$

$-0.298139$

$-0.288107$

$-0.278075$

$-0.268043$

$-0.258011$

$-0.247979$

$-0.237946$

$-0.227914$

$-0.217882$

$-0.207850$

$-0.197818$

$-0.187786$

$-0.177754$

$-0.167722$

$-0.157690$

$-0.147658$

$-0.137626$

$-0.127593$

$-0.117561$

$-0.107529$
0.396213

0.399599

0.402985

0.406371

0.409757

0.413143

0.416529

0.419915

0.423302

0.426588

0.430074

0.433460

0.436846

0.440232

0.443618

0.447004

0.450390

0.453776

0.457162

0.460548

0.463934

0.467320

0.470706

0.474092

0.477478

0.480864

0.484250

0.487636

0.491022

0.494408

0.497794

0.501180

0.504566

0.507952

0.511338

0.514724
0.935000

0.935000

0.935000

0.935000

0.935000

0.935000

0.935000

0.935000

0.935000

0.935000

0.935000

0.935000

0.935000

0.935000

0.935000

0.935000

0.935000

0.935000

0.935000

0.935000

0.935000

0.935000

0.935000

0.935000

0.935000

0.935000

0.935000

0.935000

0.935000

0.935000

0.935000

0.935000

0.935000

0.935000

0.935000

0.935000

0.935000

0.935000

0.935000 


\begin{tabular}{|c|c|c|c|c|c|}
\hline 97497 & 0.518110 & 0.935000 & 0.333883 & 0.663710 & 0.935000 \\
\hline .087465 & 0.521496 & 0.935000 & 0.343915 & 0.667096 & 0.935000 \\
\hline .077433 & 0.524882 & 0.935000 & 0.353947 & 0.670482 & 0.935000 \\
\hline .067401 & 0.528268 & .935000 & 0.363979 & 0.673868 & 0.935000 \\
\hline .057369 & 0.531654 & 0.935000 & 0.374011 & 0.677254 & 0.935000 \\
\hline 7337 & 0.535041 & 0.935000 & 0.384043 & 0.680640 & 0.935000 \\
\hline 7305 & 8427 & 0.935000 & 0.394075 & 0.684026 & 0.935000 \\
\hline & 1813 & 0.935000 & 0.404107 & 0.687412 & 0.935000 \\
\hline 241 & 199 & 0.935000 & 0.414139 & 0.690798 & 0.935000 \\
\hline .007208 & 0.548585 & .935000 & 0.424171 & 0.694184 & 0.935000 \\
\hline 0.002824 & 0.55 & 000 & 34203 & 0.697570 & 0.935000 \\
\hline 0.012856 & 357 & 000 & 4235 & 0.700956 & 0.935000 \\
\hline 0.022888 & 0.558743 & 000 & 268 & 0.704342 & 0.935000 \\
\hline 0.032920 & 0.562129 & .935000 & 300 & 0.707728 & 0.935000 \\
\hline 0.042952 & 0.565 & 000 & 332 & 0.711114 & 0.935000 \\
\hline 0.052984 & 0.568901 & 000 & 364 & 14500 & 0.935000 \\
\hline 0.063016 & 0.57 & $0.9 s$ & 4396 & 0.717886 & 0.935000 \\
\hline 0.073048 & 0.5 & 0.9 & 428 & 0.721272 & 0.935000 \\
\hline 0.083080 & 0.57 & 0.9 & 460 & 4658 & 0.935000 \\
\hline 0.093112 & 0.582445 & 0.935000 & 4492 & 0.728044 & 0.935000 \\
\hline 0.103145 & & & 24 & 430 & 0.935000 \\
\hline 177 & & & & 816 & 35000 \\
\hline 0.123 & 0.5 & & & 202 & 000 \\
\hline 0.13 & $0.5 s$ & & & 588 & \\
\hline 0.14 & 0.59 & & & 974 & \\
\hline 0.15 & 0.60 & & & 360 & \\
\hline 0.16 & 0.6 & & & 746 & \\
\hline 0.173 & 0.60 & & & 132 & 0.935000 \\
\hline 0.183401 & 0.612919 & 0.98 & & 3519 & 0.935000 \\
\hline 0.193433 & 0.616305 & 0.935000 & 813 & 1905 & 0.935000 \\
\hline 0.203465 & 0.619691 & 0.935000 & 0.634845 & 0.765291 & 0.935000 \\
\hline 0.213497 & 0.623077 & 0.935000 & 0.644877 & 0.768677 & 0.935000 \\
\hline 0.223530 & 0.626463 & 0.935000 & 0.654909 & 0.772063 & 0.935000 \\
\hline 0.233562 & 0.629849 & 0.935000 & 0.664941 & 0.775449 & 0.935000 \\
\hline 0.243594 & 0.633235 & 0.935000 & 0.674973 & 0.778835 & 0.935000 \\
\hline 0.253626 & 0.636621 & 0.935000 & 0.685006 & 0.782221 & 0.935000 \\
\hline 0.263658 & 0.640007 & 0.935000 & 0.695038 & 0.785607 & 0.935000 \\
\hline 0.273690 & 0.643393 & 0.935000 & 0.705070 & 0.788993 & 0.935000 \\
\hline 0.283722 & 0.646780 & 0.935000 & 0.715102 & 0.792379 & 0.935000 \\
\hline 0.293754 & 0.650166 & 0.935000 & 0.725134 & 0.795765 & 0.935000 \\
\hline 0.303786 & 0.653552 & 0.935000 & 0.735166 & 0.799151 & 0.935000 \\
\hline 0.313818 & 0.656938 & 0.935000 & 0.745198 & 0.802537 & 0.935000 \\
\hline 0.323850 & 0.660324 & 0.935000 & 0.755230 & 0.805923 & 0.935000 \\
\hline
\end{tabular}




$\begin{array}{llllll}0.765262 & 0.809309 & 0.935000 & 0.845519 & 0.836397 & 0.935000 \\ 0.775294 & 0.812695 & 0.935000 & 0.855551 & 0.839783 & 0.935000 \\ 0.785326 & 0.816081 & 0.935000 & 0.865583 & 0.843169 & 0.935000 \\ 0.795359 & 0.819467 & 0.935000 & 0.875615 & 0.846555 & 0.935000 \\ 0.805391 & 0.822853 & 0.935000 & 0.885647 & 0.849941 & 0.935000 \\ 0.815423 & 0.826239 & 0.935000 & 0.895679 & 0.853327 & 0.935000 \\ 0.825455 & 0.829625 & 0.935000 & 0.905712 & 0.856713 & 0.935000 \\ 0.835487 & 0.833011 & 0.935000 & 0.915744 & 0.860099 & 0.935000\end{array}$

D.2 Trajectory 2 328

$\begin{array}{llllll}-0.880000 & 0.254000 & 0.935000 & -0.559973 & 0.365627 & 0.935000 \\ -0.869999 & 0.257488 & 0.935000 & -0.549972 & 0.369115 & 0.935000 \\ -0.859998 & 0.260977 & 0.935000 & -0.539972 & 0.372604 & 0.935000 \\ -0.849997 & 0.264465 & 0.935000 & -0.529971 & 0.376092 & 0.935000 \\ -0.839997 & 0.267953 & 0.935000 & -0.519970 & 0.379580 & 0.935000 \\ -0.829996 & 0.271442 & 0.935000 & -0.509969 & 0.383069 & 0.935000 \\ -0.819995 & 0.274930 & 0.935000 & -0.499968 & 0.386557 & 0.935000 \\ -0.809994 & 0.278418 & 0.935000 & -0.489967 & 0.390045 & 0.935000 \\ -0.799993 & 0.281907 & 0.935000 & -0.479966 & 0.393534 & 0.935000 \\ -0.789992 & 0.285395 & 0.935000 & -0.469966 & 0.397022 & 0.935000 \\ -0.779992 & 0.288883 & 0.935000 & -0.459965 & 0.400510 & 0.935000 \\ -0.769991 & 0.292372 & 0.935000 & -0.449964 & 0.403999 & 0.935000 \\ -0.759990 & 0.295860 & 0.935000 & -0.439963 & 0.407487 & 0.935000 \\ -0.749989 & 0.299348 & 0.935000 & -0.429962 & 0.410975 & 0.935000 \\ -0.739988 & 0.302837 & 0.935000 & -0.419961 & 0.414464 & 0.935000 \\ -0.729987 & 0.306325 & 0.935000 & -0.409961 & 0.417952 & 0.935000 \\ -0.719987 & 0.309813 & 0.935000 & -0.399960 & 0.421440 & 0.935000 \\ -0.709986 & 0.313302 & 0.935000 & -0.389959 & 0.424929 & 0.935000 \\ -0.699985 & 0.316790 & 0.935000 & -0.379958 & 0.428417 & 0.935000 \\ -0.689984 & 0.320278 & 0.935000 & -0.369957 & 0.431905 & 0.935000 \\ -0.679983 & 0.323767 & 0.935000 & -0.359956 & 0.435394 & 0.935000 \\ -0.669982 & 0.327255 & 0.935000 & -0.349956 & 0.438882 & 0.935000 \\ -0.659982 & 0.330744 & 0.935000 & -0.339955 & 0.442370 & 0.935000 \\ -0.649981 & 0.334232 & 0.935000 & -0.329954 & 0.445859 & 0.935000 \\ -0.639980 & 0.337720 & 0.935000 & -0.319953 & 0.449347 & 0.935000 \\ -0.629979 & 0.341209 & 0.935000 & -0.309952 & 0.452835 & 0.935000 \\ -0.619978 & 0.344697 & 0.935000 & -0.299951 & 0.456324 & 0.935000 \\ -0.609977 & 0.348185 & 0.935000 & -0.289951 & 0.459812 & 0.935000 \\ -0.599977 & 0.351674 & 0.935000 & -0.279950 & 0.463300 & 0.935000 \\ -0.589976 & 0.355162 & 0.935000 & -0.269949 & 0.466789 & 0.935000 \\ -0.579975 & 0.358650 & 0.935000 & -0.259948 & 0.470277 & 0.935000 \\ -0.569974 & 0.362139 & 0.935000 & -0.249947 & 0.473766 & 0.935000\end{array}$




\begin{tabular}{|c|c|c|c|c|c|}
\hline-0.239946 & 0.477254 & 0.935000 & 0.190090 & 0.627253 & 0.935000 \\
\hline-0.229946 & 0.480742 & 0.935000 & 0.200091 & 0.630741 & 0.935000 \\
\hline 0.219945 & 0.484231 & 0.935000 & 0.210091 & 0.634229 & 0.935000 \\
\hline .209944 & 0.487719 & 0.935000 & 0.220092 & 0.637718 & 0.935000 \\
\hline 0.199943 & 0.491207 & 0.935000 & 0.230093 & 0.641206 & 0.935000 \\
\hline-0.189942 & 0.494696 & 0.935000 & 0.240094 & 0.644694 & 0.935000 \\
\hline-0.179941 & 0.498184 & 0.935000 & 0.250095 & 0.648183 & 0.935000 \\
\hline-0.169940 & 0.501672 & 0.935000 & 0.260096 & 0.651671 & 0.935000 \\
\hline-0.159940 & 0.505161 & 0.935000 & 0.270096 & 0.655159 & 0.935000 \\
\hline-0.149939 & 0.508649 & 0.935000 & 0.280097 & 0.658648 & 0.935000 \\
\hline-0.139938 & 0.512137 & 0.935000 & 0.290098 & 0.662136 & 0.935000 \\
\hline-0.129937 & 0.515626 & 0.935000 & 0.300099 & 0.665624 & 0.935000 \\
\hline-0.119936 & 0.519114 & 0.935000 & 0.310100 & 0.669113 & 0.935000 \\
\hline-0.109935 & 0.522602 & 0.935000 & 0.320101 & 0.672601 & 0.935000 \\
\hline-0.099935 & 0.526091 & 0.935000 & 0.330101 & 0.676089 & 0.935000 \\
\hline-0.089934 & 0.529579 & 0.935000 & 0.340102 & 0.679578 & 0.935000 \\
\hline-0.079933 & 0.533067 & 0.935000 & 0.350103 & 0.683066 & 0.935000 \\
\hline-0.069932 & 0.536556 & 0.935000 & 0.360104 & 0.686554 & 0.935000 \\
\hline-0.059931 & 0.540044 & 0.935000 & 0.3701 .05 & 0.690043 & 0.935000 \\
\hline-0.049930 & 0.543532 & 0.93 & 0.380106 & 0.693531 & 0.935000 \\
\hline-0.039930 & 0.547021 & 0.93 & 0.390106 & 0.697019 & 0.935000 \\
\hline-0.029929 & 0.550509 & 0.935000 & 0.400107 & 0.700508 & 0.935000 \\
\hline-0.019928 & 0.553997 & 0.935000 & 0.410108 & 0.703996 & 0.935000 \\
\hline-0.009927 & 0.557486 & 000 & 0.420109 & 0.707484 & 35000 \\
\hline 0.000074 & 0.560974 & 0.98 & 110 & 73 & 000 \\
\hline 0.010075 & 0.564 & 0.9 & & 61 & \\
\hline 0.020075 & 0.567 & 0.9 & 11 & 0.717949 & 000 \\
\hline 0.030076 & 0.57 & 0.9 & & 21438 & 000 \\
\hline 0.040077 & 0.57 & 0.9 & 13 & 0.724926 & 000 \\
\hline 0.050078 & 0.57 & 0.9 & & 8414 & 000 \\
\hline 0.060079 & 0.58 & & & 0.731903 & 000 \\
\hline 0.070080 & 0.585392 & 0.9 & 16 & 0.735391 & 000 \\
\hline 0.080080 & 0.588881 & 00 & & 0.738879 & 000 \\
\hline 0.090081 & 0.592369 & 00 & & 0.742368 & 35000 \\
\hline 0.100082 & 0.595857 & 0.93 & & 0.745856 & 000 \\
\hline 0.110083 & 0.599346 & 0.935000 & 0.540119 & 0.749344 & 0.935000 \\
\hline 0.120084 & 0.602834 & 0.935000 & 0.550120 & 0.752833 & 0.935000 \\
\hline 0.130085 & 0.606322 & 0.935000 & 0.560121 & 0.756321 & 0.935000 \\
\hline 0.140085 & 0.609811 & 0.935000 & 0.570122 & 0.759810 & 0.935000 \\
\hline 0.150086 & 0.613299 & 0.935000 & 0.580122 & 0.763298 & 0.935000 \\
\hline 0.160087 & 0.616788 & 0.935000 & 0.590123 & 0.766786 & 0.835000 \\
\hline 0.170088 & 0.620276 & 0.935000 & 0.600124 & 0.770275 & 0.935000 \\
\hline 0.180089 & 0.623764 & 0.935000 & 0.610125 & 0.773763 & 0.935000 \\
\hline
\end{tabular}




\begin{tabular}{|c|c|c|c|c|c|}
\hline 0.620126 & 0.777251 & 0.935000 & 1.050162 & 0.927250 & 0.935000 \\
\hline 0.630127 & 0.780740 & 0.935000 & 1.060163 & 0.930738 & 35000 \\
\hline 640127 & 0.784228 & 0.935000 & .070163 & 0.934227 & 935000 \\
\hline 0.650128 & 0.787716 & 0.935000 & .080164 & 0.937715 & ח \\
\hline 0.660129 & 0.791205 & 0.935000 & .090165 & 0.941203 & 500 \\
\hline 0.670130 & 0.794693 & 0.935000 & .100166 & 0.944692 & \\
\hline 0.680131 & 0.798181 & 0.935000 & .110167 & 0.948180 & o \\
\hline 0.690132 & 0.801670 & 0.935000 & .120168 & 0.951668 & \\
\hline 0.700132 & 0.805158 & 0.935000 & 1.130168 & 0.955157 & \\
\hline 0.7101 & 0.808646 & 5000 & .140169 & 0.958645 & \\
\hline 0.7 & & 5000 & 50170 & 0.962133 & \\
\hline 35 & 0.815623 & 000 & 0171 & 0.965622 & \\
\hline 36 & 19111 & 5000 & .170172 & 0.969110 & \\
\hline 0.750137 & 0.822600 & 0.935000 & .180173 & 0.972598 & \\
\hline 0.760137 & 0.826088 & 0.935000 & .190173 & 0.976087 & \\
\hline 0.770138 & 0.829576 & 000 & .200174 & 0.979575 & \\
\hline 30139 & 0.833 & 35000 & 0175 & 0.983063 & \\
\hline 0.790140 & 0.836 & 0.935000 & 1.220176 & 0.986552 & \\
\hline 0.800141 & 0.840041 & 0.935000 & 1.230177 & 0.990040 & \\
\hline 0.810142 & 0.843530 & 0.935000 & 0178 & 0.993528 & \\
\hline 0142 & 847018 & 35000 & 79 & 0.997017 & \\
\hline 0.830143 & 0.8505 & 0.935000 & 1.2601 & 1.00050 & \\
\hline 0.840144 & 0.853995 & 0.935000 & 80 & 1.0039 & \\
\hline 50145 & 0.857483 & 0.935000 & 81 & 1.007482 & \\
\hline 0.860146 & 0.860971 & 0.935000 & 1.290182 & 1.010970 & 0.93 \\
\hline 0.870147 & 0.864460 & 0.935000 & 1.300183 & 1.014458 & 0.9 \\
\hline 0.880148 & 0.867948 & 0.935000 & 84 & 1.017947 & \\
\hline 0148 & 871436 & 35000 & & & \\
\hline 0.900149 & 0.874925 & 0.935000 & 1.330185 & 1.024923 & 0.9 \\
\hline 0.910150 & 0.878413 & 0.935000 & 1.340186 & 1.028412 & 0.9 \\
\hline 0.920151 & 0.881901 & 0.935000 & 1.350187 & 1.031900 & 0.9 \\
\hline 0.930152 & 0.885390 & 0.935000 & 1.360188 & 1.035388 & \\
\hline 0.940153 & 0.888878 & 0.935000 & 1.370189 & 1.038877 & \\
\hline 0.950153 & 0.892366 & 0.935000 & 1.380189 & 1.042365 & 0.9350 \\
\hline 0.960154 & 0.895855 & 0.935000 & 1.390190 & 1.045854 & 0.9350 \\
\hline 0.970155 & 0.899343 & 0.935000 & 1.400191 & 1.049342 & 0.9 \\
\hline 0.980156 & 0.902832 & 0.935000 & 1.410192 & 1.052830 & 0.93500 \\
\hline 0.990157 & 0.906320 & 0.935000 & 1.420193 & 1.056319 & 0.935000 \\
\hline 1.000158 & 0.909808 & 0.935000 & 1.430194 & 1.059807 & 0.935000 \\
\hline 1.010158 & 0.913297 & 0.935000 & 1.440194 & 1.063295 & 0.93500 \\
\hline 1.020159 & 0.916785 & 0.935000 & 1.450195 & 1.066784 & 0.93500 \\
\hline 1.030160 & 0.920273 & 0.935000 & 1.460196 & 1.070272 & 0.93500 \\
\hline 1.040161 & 0.923762 & 0.935000 & 1.470197 & 1.073760 & 0.93500 \\
\hline
\end{tabular}




\begin{tabular}{|c|c|c|c|c|c|}
\hline 1.480198 & 1.077249 & $0: 935000$ & 1.910234 & 1.227247 & 0.935000 \\
\hline 1.490199 & 1.080737 & 0.935000 & 1.920235 & 1.230736 & 0.935000 \\
\hline .500199 & 1.084225 & 0.935000 & 1.930235 & 1.234224 & 0.935000 \\
\hline .510200 & 1.087714 & 0.935000 & 1.940236 & 1.237712 & 0.935000 \\
\hline 520201 & 1.091202 & 0.935000 & 1.950237 & 1.241201 & 0.935000 \\
\hline 1.530202 & 1.094690 & 0.935000 & 1.960238 & 1.244689 & 0.935000 \\
\hline 1.540203 & 1.098179 & 0.935000 & 1.970239 & 1.248177 & 0.935000 \\
\hline 1.550204 & 1.101667 & 0.935000 & 1.980240 & 1.251666 & 0.935000 \\
\hline 1.560204 & 1.105155 & 0.935000 & 1.990241 & 1.255154 & 0.935000 \\
\hline 1.570205 & 1.108644 & 0.935000 & 2.000241 & 1.258642 & 0.935000 \\
\hline 1.580206 & 1.112132 & 0.935000 & 2.010242 & 1.262131 & 0.935000 \\
\hline 1.590207 & 1.115620 & 0.935000 & 2.020243 & 1.265619 & 0.935000 \\
\hline 1.600208 & 1.119109 & 0.935000 & 2.030244 & 1.269107 & 0.935000 \\
\hline 1.610209 & 1.122597 & 0.935000 & 2.040246 & 1.272596 & 0.935000 \\
\hline 1.620210 & 1.126085 & 0.935000 & 2.050246 & 1.276084 & 0.935000 \\
\hline 1.630210 & 1.129574 & 0.935000 & 2.060246 & 1.279572 & 0.935000 \\
\hline 1.640211 & 1.133062 & 0.935000 & 2.070247 & 1.283061 & 0.935000 \\
\hline 1.650212 & 1.136550 & 0.935000 & 2.080248 & 1.286549 & 0.935000 \\
\hline 1.660213 & 1.140039 & 0.935000 & 2.090249 & 1.290037 & 0.935000 \\
\hline 1.670214 & 1.143527 & 0.935000 & 2.100250 & 1.293526 & 0.935000 \\
\hline 1.680215 & 1.147015 & 0.935000 & 2.110251 & 1.297014 & 0.935000 \\
\hline 1.690215 & 1.150504 & 15000 & 2.120251 & 1.300502 & 0.935000 \\
\hline 1.700216 & 1.153992 & 0.935000 & 2.130252 & 1.303991 & 0.935000 \\
\hline 1.710217 & 1.157480 & 0.935000 & 2.140253 & 1.307479 & 0.935000 \\
\hline 1.720218 & 1.160969 & 0.935000 & 2.150254 & 1.310967 & 0.935000 \\
\hline $1.73 c$ & 1.164457 & 5000 & 2.160255 & 1.314456 & 0.935000 \\
\hline 1.74 & 1.167945 & 5000 & 256 & 1.317944 & 0.935000 \\
\hline 1.75 & 1.171434 & 5000 & 0256 & 1.321432 & 0.935000 \\
\hline 1.760 & 1.174 & & 257 & 1.324 & 0.935000 \\
\hline 1.77 & 1.178410 & 0.9 & 0258 & 1.328409 & 0.935000 \\
\hline 1.780223 & 1.181899 & 0.935000 & 2.210259 & 1.331898 & 0.935000 \\
\hline 1.790224 & 1.185387 & 0.935000 & 2.220260 & 1.335386 & 0.935000 \\
\hline 1.800225 & 1.188876 & 0.935000 & 2.230261 & 1.338874 & 0.935000 \\
\hline 1.810225 & 1.192364 & 0.935000 & 2.240261 & 1.342363 & 0.935000 \\
\hline 1.820226 & 1.195852 & 0.935000 & 2.250262 & 1.345851 & 0.935000 \\
\hline 1.830227 & 1.199341 & 0.935000 & 2.260263 & 1.349339 & 0.935000 \\
\hline 1.840228 & 1.202829 & 0.935000 & 2.270264 & 1.352828 & 0.935000 \\
\hline 1.850229 & 1.206317 & 0.935000 & 2.280265 & 1.356316 & 0.935000 \\
\hline 1.860230 & 1.209806 & 0.935000 & 2.290266 & 1.359804 & 0.935000 \\
\hline 1.870230 & 1.213294 & 0.935000 & 2.300267 & 1.363293 & 0.935000 \\
\hline 1.880231 & 1.216782 & 0.935000 & 2.310257 & 1.356781 & 0.935000 \\
\hline 1.890232 & 1.220271 & 0.935000 & 2.320268 & 1.370269 & 0.935000 \\
\hline 1.900233 & 1.223759 & 0.935000 & 2.330269 & 1.373758 & 0.935000 \\
\hline
\end{tabular}




$\begin{array}{llllll}2.340270 & 1.377246 & 0.935000 & 2.370272 & 1.387711 & 0.935000 \\ 2.350271 & 1.380734 & 0.935000 & 2.380273 & 1.391199 & 0.935000 \\ 2.360272 & 1.384223 & 0.935000 & 2.390274 & 1.394688 & 0.935000\end{array}$

D.3 Trajectory 3

186

\begin{tabular}{|c|c|c|c|c|c|}
\hline 0.880000 & 0.254000 & 0.935000 & -0.623618 & -0.116799 & 0.656392 \\
\hline-0.873071 & 0.243978 & 0.927470 & -0.616689 & -0.126821 & 0.648862 \\
\hline .866142 & 0.233957 & 0.919940 & -0.609759 & -0.136843 & 0.641332 \\
\hline-0.859212 & 0.223935 & 0.912410 & -0.602830 & -0.146864 & 0.633802 \\
\hline .852283 & 0.213914 & 0.904880 & -0.595901 & -0.156886 & 0.626272 \\
\hline .845354 & 0.203892 & 0.897350 & -0.588972 & -0.166907 & 0.618742 \\
\hline-0.838425 & 0.193870 & 0.889820 & -0.582042 & -0.176929 & 0.611212 \\
\hline$=0.831495$ & 0.183849 & 0.882290 & -0.575113 & -0.186951 & 0.603682 \\
\hline-0.824566 & 0.173827 & 0.874760 & -0.568184 & -0.196972 & 0.596152 \\
\hline-0.817637 & 0.163806 & 0.867230 & -0.561255 & -0.206994 & 0.588622 \\
\hline-0.810708 & 0.153784 & 0.859700 & -0.554326 & -0.217015 & 0.581092 \\
\hline-0.803778 & 0.143762 & 0.852170 & -0.547396 & -0.227037 & 0.573562 \\
\hline-0.796849 & 0.133741 & 0.844641 & -0.540467 & -0.237059 & 0.566032 \\
\hline-0.789920 & 0.123719 & 0.837111 & -0.533538 & -0.247080 & 0.558502 \\
\hline-0.782991 & 0.113698 & 0.829581 & -0.526609 & -0.257102 & 0.550972 \\
\hline-0.776061 & 0.103676 & 0.822051 & 679 & -0.267123 & 43442 \\
\hline-0.769132 & 0.093654 & 0.81 & 750 & -0.277145 & 0.535912 \\
\hline-0.762203 & 0.083633 & 0.806991 & 821 & -0.287167 & 0.528382 \\
\hline-0.755274 & 0.073611 & 0.799461 & 892 & -0.297188 & 0.520852 \\
\hline-0.748344 & 0.063590 & 0.791931 & -0.491962 & -0.307210 & 0.513322 \\
\hline-0.741415 & 0.053568 & 0.784401 & -0.485033 & -0.317231 & 0.505792 \\
\hline-0.734486 & 0.043546 & 0.776871 & -0.478104 & -0.327253 & 0.498263 \\
\hline-0.727557 & 0.033525 & 0.769341 & -0.471175 & -0.337275 & 0.490733 \\
\hline 0.720627 & 0.023503 & 0.761811 & -0.464245 & -0.347296 & 0.483203 \\
\hline 0.713698 & 0.013482 & 0.754281 & -0.457316 & -0.357318 & 0.475673 \\
\hline $\begin{array}{r}0.706769 \\
-0.699840\end{array}$ & $\begin{array}{r}0.003460 \\
-0.006562\end{array}$ & & $\begin{array}{l}-0.450387 \\
-0.443458\end{array}$ & $\begin{array}{l}-0.367339 \\
-0.377361\end{array}$ & 0.468143 \\
\hline 0.692910 & -0.016583 & 0.731691 & -0.436528 & -0.387383 & 0.453083 \\
\hline 0.685981 & -0.026605 & 0.724161 & -0.429599 & -0.397404 & 0.445553 \\
\hline 0.679052 & -0.036627 & 0.716631 & -0.422670 & -0.407426 & 0.438023 \\
\hline-0.672123 & -0.046648 & 0.709101 & -0.415741 & -0.417447 & 0.43049 \\
\hline-0.665193 & -0.056670 & 0.701571 & -0.408811 & -0.427469 & 0.42296 \\
\hline 0.658264 & -0.066691 & 0.694041 & -0.401882 & -0.437491 & 0.415433 \\
\hline-0.651335 & -0.076713 & 0.686511 & -0.394953 & -0.447512 & 0.407903 \\
\hline-0.644406 & -0.086735 & 0.678981 & -0.388024 & -0.457534 & 0.400373 \\
\hline-0.637476 & -0.096756 & 0.671452 & -0.381094 & -0.467556 & 0.392843 \\
\hline-0.630547 & -0.106778 & 0.663922 & -0.374165 & -0.477577 & 0.385313 \\
\hline
\end{tabular}




\begin{tabular}{|c|c|c|c|c|c|}
\hline-0.367236 & -0.487599 & 0.377783 & -0.069278 & -0.918528 & 0.053995 \\
\hline-0.360307 & -0.497620 & 0.370253 & -0.062349 & -0.928549 & 0.046465 \\
\hline-0.353377 & -0.507642 & 0.362723 & -0.055420 & -0.938571 & 0.038935 \\
\hline-0.346448 & -0.517664 & 0.355193 & -0.048491 & -0.948593 & 0.031405 \\
\hline-0.339519 & -0.527685 & 0.347663 & -0.041561 & -0.958614 & 0.02387 \\
\hline-0.332590 & -0.537707 & 0.340133 & -0.034632 & -0.968636 & 0.016345 \\
\hline-0.325660 & -0.547728 & 0.332603 & -0.027703 & -0.978657 & 0.008815 \\
\hline-0.318731 & -0.557750 & 0.325074 & -0.020774 & -0.988679 & 0.001285 \\
\hline-0.311802 & -0.567772 & 0.317544 & -0.013844 & -0.998701 & -0.006245 \\
\hline-0.304873 & -0.577793 & 0.310014 & -0.006915 & -1.008722 & -0.013775 \\
\hline-0.297943 & -0.587815 & 0.302484 & 0.000014 & -1.018744 & -0.02 \\
\hline-0.291014 & -0.597836 & 0.294954 & 0.006943 & -1.028765 & -0.02 \\
\hline-0.284085 & -0.607858 & 0.287424 & 0.013873 & -1.038787 & $-0.03 t$ \\
\hline-0.277156 & -0.617880 & 0.279894 & 0.020802 & -1.048809 & -0.043894 \\
\hline-0.270227 & -0.627901 & 0.272364 & 0.027731 & -1.058830 & -0.05 \\
\hline-0.263297 & -0.637923 & 0.264834 & 0.034660 & -1.068852 & -0.058954 \\
\hline-0.256368 & -0.647944 & 0.257304 & 0.041589 & -1.078873 & -0.066484 \\
\hline-0.249439 & -0.657966 & 0.249774 & 0.048519 & -1.088895 & -0.074014 \\
\hline-0.242510 & -0.667988 & 0.242244 & 0.055448 & -1.098917 & -0.081544 \\
\hline-0.235580 & -0.678009 & 0.234714 & 0.062377 & -1.108938 & -0.089074 \\
\hline-0.228651 & -0.688031 & 0.227184 & 0.069306 & -1.118960 & -0.096604 \\
\hline-0.221722 & -0.698052 & 0.219654 & 0.076236 & -1.128981 & -0.104134 \\
\hline-0.214793 & -0.708074 & 0.212124 & 0.083165 & -1.139003 & -0.111664 \\
\hline-0.207863 & -0.718096 & 0.204594 & 0.090094 & -1.149025 & -0.119194 \\
\hline-0.200934 & -0.728117 & 0.197064 & 0.097023 & -1.159046 & -0.126724 \\
\hline-0.194005 & -0.738139 & 0.189534 & 0.103953 & -1.169068 & -0.134254 \\
\hline-0.187076 & -0.748160 & 0.182004 & 0.110882 & -1.179089 & -0.141784 \\
\hline-0.180146 & -0.758182 & 0.174474 & 0.117811 & -1.189111 & -0.149314 \\
\hline-0.173217 & -0.768204 & 0.166944 & 0.124740 & -1.199133 & -0.156844 \\
\hline-0.166288 & -0.778225 & 0.159414 & 0.131670 & -1.209154 & -0.164374 \\
\hline-0.159359 & -0.788247 & 0.151885 & 0.138599 & -1.219176 & -0.171904 \\
\hline-0.152429 & -0.798268 & 0.144355 & 0.145528 & -1.229197 & -0.179434 \\
\hline-0.145500 & -0.808290 & 0.136825 & 0.152457 & -1.239219 & -0.186964 \\
\hline-0.138571 & -0.818312 & 0.129295 & 0.159387 & -1.249241 & -0.194493 \\
\hline-0.131642 & -0.828333 & 0.121765 & 0.166316 & -1.259262 & -0.202023 \\
\hline-0.124712 & -0.838355 & 0.114235 & 0.173245 & -1.269284 & -0.209553 \\
\hline-0.117783 & -0.848376 & 0.106705 & 0.180174 & -1.279305 & -0.217083 \\
\hline-0.110854 & -0.858398 & 0.099175 & 0.187104 & -1.289327 & -0.224613 \\
\hline-0.103925 & -0.868420 & 0.091645 & 0.194033 & -1.299349 & -0.232143 \\
\hline-0.096995 & -0.878441 & 0.084115 & 0.200962 & -1.309370 & -0.239673 \\
\hline-0.090066 & -0.888463 & 0.076585 & 0.207891 & -1.313392 & -0.247203 \\
\hline-0.083137 & -0.898484 & 0.069055 & 0.214821 & -1.329413 & -0.254733 \\
\hline-0.076208 & -0.908506 & 0.061525 & 0.221750 & -1.339435 & -0.262263 \\
\hline
\end{tabular}



$\begin{array}{llll}0.228679 & -1.349457 & -0.269793\end{array}$
$0.235608-1.359478-0.277323$
$0.242538-1.369500-0.284853$
$0.249467-1.379522-0.292383$
$\begin{array}{llll}0.256396 & -1.389543 & -0.299913\end{array}$
$0.263325-1.399565-0.307443$
$\begin{array}{llll}0.270255 & -1.409586 & -0.314973\end{array}$
$0.277184-1.419608-0.322503$
$0.284113-1.429630-0.330033$
$0.291042-1.439651-0.337563$
$0.297972-1.449673-0.345093$
$0.304901-1.459694-0.352623$
$\begin{array}{llll}0.311830 & -1.469716 & -0.360153\end{array}$
$0.318759-1.479738=0.367682$
$\begin{array}{llll}0.325688 & -1.489759 & -0.375212\end{array}$
$0.332618-1.499781-0.382742$
$\begin{array}{llll}0.339547 & -1.509802 & -0.390272\end{array}$
$\begin{array}{llll}0.346476 & -1.519824 & -0.397802\end{array}$
$\begin{array}{llll}0.353405 & -1.529846 & -0.405332\end{array}$
$\begin{array}{llll}0.360335 & -1.539867 & -0.412862\end{array}$
$\begin{array}{llll}0.367264 & -1.549889 & -0.420392\end{array}$
$\begin{array}{llll}0.374193 & -1.559910 & -0.427922\end{array}$
$\begin{array}{llll}0.381122 & -1.569932 & -0.435452\end{array}$
$\begin{array}{llll}0.388052 & -1.579954 & -0.442982\end{array}$
$\begin{array}{llll}0.394981 & -1.589975 & -0.450512\end{array}$
$\begin{array}{llll}0.401910 & -1.599997 & -0.458042\end{array}$

\section{D.4 Trajectory 4}

$\begin{array}{ll}-0.880000 & 0.254000 \\ -0.869974 & 0.256510 \\ -0.859948 & 0.259020 \\ -0.849922 & 0.261530 \\ -0.839896 & 0.264040 \\ -0.829870 & 0.266550 \\ -0.819843 & 0.269060 \\ -0.809817 & 0.271570 \\ -0.799791 & 0.274080 \\ -0.789765 & 0.276590 \\ -0.779739 & 0.279100 \\ -0.769713 & 0.281610 \\ -0.759687 & 0.284120 \\ -0.749661 & 0.286630 \\ -0.739635 & 0.289140 \\ -0.729609 & 0.291650 \\ -0.719583 & 0.294160 \\ -0.709556 & 0.296670 \\ -0.699530 & 0.299180 \\ -0.689504 & 0.301690 \\ -0.679478 & 0.304200 \\ -0.669452 & 0.306710 \\ -0.659426 & 0.309220 \\ -0.649400 & 0.311730 \\ -0.639374 & 0.314240 \\ -0.629348 & 0.316750 \\ -0.619322 & 0.319260\end{array}$

0.935000

0.930457

0.925913

0.921370

0.916826

0.912283

0.907739

0.903196

0.898652

0.894109

0.889565

0.885022

0.880478

0.875935

0.871391

0.866848

0.862304

0.857761

0.853217

0.848674

0.844130

0.839587

0.835043

0.830500

0.825956

0.821413

0.816869
$-0.609296$

$-0.599270$

$-0.589243$

$-0.579217$

$-0.569191$

$-0.559165$

$-0.549139$

$-0.539113$

$-0.529087$

$-0.519061$

$-0.509035$

$-0.499009$

$-0.488983$

$-0.478956$

$-0.468930$

$-0.458904$

$-0.448878$

$-0.438852$

$-0.428826$

$-0.418800$

$-0.408774$

$-0.398748$

$-0.388722$

$-0.378696$

$-0.368669$

$-0.358643$

$-0.348617$
0.321770

0.324280

0.326790

0.329300

0.331810

0.334320

0.336830

0.339340

0.341850

0.344360

0.346870

0.349380

0.351890

0.354400

0.356910

0.359420

0.361930

0.364440

0.366950

0.369460

0.371970

0.374480

0.376990

0.379500

0.382010

0.384520

0.387030
0.812326

0.807782

0.803239

0.798695

0.794152

0.789608

0.785065

0.780521

0.775978

0.771434

0.766891

0.762847

0.757804

0.753260

0.748717

0.744173

0.739630

0.735086

0.730543

0.725999

0.721456

0.716912

0.712369

0.707825

0.703282

0.698738

0.694195 


\begin{tabular}{|c|c|c|c|c|c|}
\hline-0.338591 & 0.389540 & 0.689651 & 0.092531 & 0.497471 & 0.494281 \\
\hline-0.328565 & 0.392050 & 0.685108 & 0.102557 & 0.499981 & 0.489737 \\
\hline-0.318539 & 0.394560 & 0.680564 & 0.112583 & 0.502491 & 0.485194 \\
\hline-0.308513 & 0.397070 & 0.676021 & 0.122609 & 0.505001 & 0.480650 \\
\hline-0.298487 & 0.399580 & 0.671477 & 0.132635 & 0.507511 & 0.476107 \\
\hline-0.288461 & 0.402090 & 0.666934 & 0.142661 & 0.510021 & 0.471563 \\
\hline-0.278435 & 0.404600 & 0.662390 & 0.152687 & 0.512531 & 0.467020 \\
\hline-0.268409 & 0.407110 & 0.657847 & 0.162713 & 0.515041 & 0.462476 \\
\hline-0.258383 & 0.409620 & 0.653303 & 0.172739 & 0.517551 & 0.457933 \\
\hline-0.248356 & 0.412130 & 0.648760 & 0.182765 & 0.520061 & 0.453389 \\
\hline-0.238330 & 0.414640 & 0.644216 & 0.192791 & 0.522571 & 0.448846 \\
\hline-0.228304 & 0.417150 & 0.639673 & 0.202818 & 0.525081 & 0.444302 \\
\hline-0.218278 & 0.419660 & 0.635129 & 0.212844 & 0.527591 & 0.439759 \\
\hline-0.208252 & 0.422170 & 0.630586 & 0.222870 & 0.530101 & 0.435215 \\
\hline-0.198226 & 0.424680 & 0.626042 & 0.232896 & 0.532611 & 0.430672 \\
\hline-0.188200 & 0.427190 & 0.621499 & 0.242922 & 0.535121 & 0.426128 \\
\hline-0.178174 & 0.429700 & 0.616955 & 0.252948 & 0.537631 & 0.421585 \\
\hline-0.168148 & 0.432210 & 0.612412 & 0.262974 & 141 & 7041 \\
\hline-0.158122 & 0.434720 & 0.607868 & 0.273000 & 42651 & 0.412498 \\
\hline-0.148096 & 0.437230 & 0.603325 & 0.283026 & 161 & 7954 \\
\hline-0.138069 & 0.439740 & 0.598781 & 0.293052 & 671 & 0.403411 \\
\hline-0.128043 & 0.442250 & 0.594238 & 3078 & 0181 & 0.398867 \\
\hline-0.118017 & 0.444760 & 694 & 105 & 0.552691 & 0.394324 \\
\hline-0.107991 & 0.447270 & 0.585151 & 0.323131 & 0.555201 & 0.389780 \\
\hline-0.097965 & 0.449780 & 0.580607 & 0.333157 & 0.557711 & 0.385237 \\
\hline-0.087939 & 0.452290 & 0.576064 & 0.343183 & 0.560221 & 0.380693 \\
\hline-0.077913 & 0.454800 & 0.571520 & 0.353209 & 0.562731 & 0.376150 \\
\hline-0.067887 & 0.457310 & 0.566977 & 0.363235 & 0.565241 & 0.371606 \\
\hline-0.057861 & 0.459820 & 0.562433 & 0.373261 & 0.567751 & 0.367063 \\
\hline-0.047835 & 0.462330 & 0.557890 & 0.383287 & 0.570261 & 0.362519 \\
\hline-0.037809 & 0.464840 & 0.553346 & 0.393313 & 0.572771 & 0.357976 \\
\hline-0.027782 & 0.467350 & 0.548803 & 0.403339 & 0.575281 & 0.353432 \\
\hline-0.01 & 0.46 & 0.5 & 365 & 0.577791 & 0.348889 \\
\hline-0.007730 & 0.472370 & 0.5 & 0.4 & 301 & 0.34 \\
\hline 0.002296 & 0.474880 & 0.535172 & 0.4 & 0.582811 & 0.339802 \\
\hline 0.012322 & 0.477390 & 0.530629 & 0.4 & 0.585321 & 0.335258 \\
\hline 0.022348 & 0.479900 & 0.526085 & 0.453470 & 0.587831 & 0.330715 \\
\hline 0.032374 & 0.482410 & 0.521542 & 0.463496 & 0.590341 & 0.326171 \\
\hline 0.042400 & 0.484920 & 0.516998 & 0.473522 & 0.592851 & 0.321628 \\
\hline 0.052426 & 0.487430 & 0.512455 & 0.483548 & 0.595361 & 0.317084 \\
\hline 0.062452 & 0.489940 & 0.507911 & 0.493574 & 0.597871 & 0.312541 \\
\hline 0.072478 & 0.492451 & 0.503368 & 0.503600 & 0.600381 & 0.307997 \\
\hline 0.082505 & 0.494961 & 0.498824 & 0.513626 & 0.602891 & 0.303454 \\
\hline
\end{tabular}




\begin{tabular}{|c|c|c|c|c|c|}
\hline 0.523652 & 0.605401 & 0.298910 & 0.954774 & 0.713331 & 0.103540 \\
\hline 0.533678 & 0.607911 & 0.294367 & 0.964800 & 0.715841 & 0.098996 \\
\hline 0.543705 & 0.610421 & 0.289823 & 0.974826 & 0.718351 & 0.094453 \\
\hline 0.553731 & 0.612931 & 0.285280 & 0.984852 & 0.720861 & 0.089909 \\
\hline 0.563757 & 0.615441 & 0.280736 & 0.994879 & 0.723371 & 0.085366 \\
\hline 0.573783 & 0.617951 & 0.276193 & 1.004905 & 0.725881 & 0.080822 \\
\hline 0.583809 & 0.620461 & 0.271649 & 1.014931 & 0.728391 & 0.076279 \\
\hline 0.593835 & 0.622971 & 0.267106 & 1.024957 & 0.730901 & 0.071735 \\
\hline 0.603861 & 0.625481 & 0.262562 & 1.034983 & 0.733411 & 0.067192 \\
\hline 0.613887 & 0.627991 & 0.258019 & 1.045009 & 0.735921 & 0.062648 \\
\hline 0.623913 & 0.630501 & 0.253475 & 1.055035 & 0.738431 & 0.058105 \\
\hline 0.633939 & 0.633011 & 0.248932 & 1.065061 & 0.740941 & 0.053561 \\
\hline 0.643965 & 0.635521 & 0.244388 & 1.075087 & 0.743451 & 0.049018 \\
\hline 0.653992 & 0.638031 & 0.239845 & 1.085113 & 0.745961 & 0.044474 \\
\hline 0.664018 & 0.640541 & 0.235301 & 1.095139 & 0.748471 & 0.039931 \\
\hline 0.674044 & 0.643051 & 0.230758 & 1.105166 & 0.750981 & 0.035387 \\
\hline 0.684070 & 0.645561 & 0.226214 & 1.115192 & 0.753491 & 0.030844 \\
\hline 0.694096 & 0.648071 & 0.221671 & 1.125218 & 0.756001 & 0.026300 \\
\hline 0.704122 & 0.650581 & 0.217127 & 1.135244 & 0.758511 & 0.021757 \\
\hline 0.714148 & 0.653091 & 0.212584 & 5270 & 021 & 213 \\
\hline 0.724174 & 0.655601 & 0.208040 & 1.155296 & 0.763531 & 0.012670 \\
\hline 0.734200 & 0.6581 & 0.203 & 1.1 & 0.766041 & 0.008126 \\
\hline 0.744226 & 0.660621 & 0.198953 & 1.17 & 8551 & 3583 \\
\hline 0.754252 & 0.663131 & 0.194410 & 5374 & 1061 & -0.000961 \\
\hline 0.764279 & 0.665641 & 0.189866 & 1.195400 & 0.773571 & -0.005504 \\
\hline 0.774305 & 0.668151 & 0.185323 & 1.205426 & 0.776081 & -0.010048 \\
\hline 0.784331 & 0.670661 & 0.180779 & 453 & 3591 & -0.014591 \\
\hline 0.794357 & 0.673171 & 0.176236 & 479 & 101 & -0.019135 \\
\hline 0.804383 & 0.675681 & 0.171692 & 505 & 611 & -0.023678 \\
\hline 0.814409 & 0.678191 & 0.167149 & 531 & 0.786121 & -0.028222 \\
\hline 0.824435 & 0.680701 & 0.162605 & 1.255557 & 0.788631 & -0.032765 \\
\hline 0.834461 & 0.683211 & 0.158062 & 1.265583 & 0.791141 & -0.037309 \\
\hline 0.844487 & 0.685721 & 0.153518 & 1.275609 & 0.793651 & -0.041852 \\
\hline 0.854513 & 0.688231 & 0.148975 & 1.285635 & 0.796161 & -0.046396 \\
\hline 0.864539 & 0.690741 & 0.144431 & 1.295661 & 0.798671 & -0.050939 \\
\hline 0.874565 & 0.693251 & 0.139888 & 1.305687 & 0.801181 & -0.055483 \\
\hline 0.884592 & 0.695761 & 0.135344 & 1.315713 & 0.803691 & -0.060026 \\
\hline 0.894618 & 0.698271 & 0.130801 & 1.325739 & 0.806201 & -0.064570 \\
\hline 0.904644 & 0.700781 & 0.126257 & 1.335766 & 0.808711 & -0.069113 \\
\hline 0.914670 & 0.703291 & 0.121714 & 1.345792 & 0.811221 & -0.073657 \\
\hline 0.924696 & 0.705801 & 0.117170 & 1.355818 & 0.813731 & -0.073200 \\
\hline 0.934722 & 0.708311 & 0.112627 & 1.365844 & 0.816241 & -0.082744 \\
\hline .0 .944748 & 0.710821 & 0.108083 & 1.375870 & 0.818751 & -0.087287 \\
\hline
\end{tabular}




\begin{tabular}{|c|c|c|c|c|c|}
\hline 1.385896 & 0.821261 & -0.091831 & 1.756861 & 0.914131 & -0.259940 \\
\hline 1.395922 & 0.823771 & -0.096374 & 1.766887 . & 0.916641 & -0.264484 \\
\hline 1.405948 & 0.826281 & -0.100918 & 1.776913 & 0.919151 & -0.269027 \\
\hline 1.415974 & 0.828791 & & 1.786940 & 0.921661 & -0.273571 \\
\hline 1.426000 & 0.831301 & -0.110005 & 1.796966 & 0.924171 & -0.278114 \\
\hline 1.436026 & 0.833811 & -0.114548 & 1.806992 & 0.926681 & -0.282658 \\
\hline 1.446053 & 0.836321 & -0.119092 & 1.817018 & 0.929191 & -0.287201 \\
\hline 1.456079 & 0.838831 & -0.123635 & 1.827044 & 0.931701 & -0.291745 \\
\hline 1.466105 & 0.841341 & -0.128179 & 1.837070 & 0.934211 & -0.296288 \\
\hline 1.476131 & 0.843851 & -0.132722 & 1.847096 & 0.936721 & -0.300832 \\
\hline 1.486157 & 0.846361 & -0.137266 & 1.857122 & 0.939231 & -0.305375 \\
\hline 1.496183 & 0.848871 & -0.141809 & 1.867148 & 0.941741 & -0.309919 \\
\hline 1.506209 & 0.851381 & -0.146353 & 1.877174 & 0.944251 & -0.31 .4462 \\
\hline 1.516235 & 0.853891 & -0.150896 & 1.887200 & 0.946761 & -0.319006 \\
\hline 1.526261 & 0.856401 & -0.155440 & 1.897227 & 0.949271 & -0.323549 \\
\hline 1.536287 & 0.858911 & -0.159983 & 1.907253 & 0.951781 & -0.328093 \\
\hline 1.546313 & 0.861421 & -0.164527 & 1.917279 & 0.954291 & -0.332636 \\
\hline 1.556340 & 0.863931 & -0.169070 & 1.927305 & 0.956801 & -0.337180 \\
\hline 1.566366 & 0.866441 & -0.173614 & 1.937331 & 0.959311 & -0.341723 \\
\hline 1.576392 & 0.868951 & -0.178157 & 1.947357 & 0.961821 & -0.346267 \\
\hline 1.586418 & 0.871461 & -0.182701 & 1.957383 & 0.964331 & -0.350810 \\
\hline 1.596444 & 0.873971 & -0.187244 & 1.967409 & 0.966841 & -0.355354 \\
\hline 1.606470 & 0.876481 & -0.191788 & 1.977435 & 0.969351 & -0.359897 \\
\hline 1.616496 & 0.878991 & -0.196331 & 1.987461 & 0.971861 & -0.364441 \\
\hline 1.626522 & 0.881501 & -0.200875 & 1.997487 & 0.974372 & -0.368984 \\
\hline 1.636548 & 0.884011 & -0.205418 & 2.007514 & 0.976882 & -0.373528 \\
\hline 1.646574 & 0.886521 & -0.209962 & 2.017540 & 0.979392 & -0.378071 \\
\hline 1.656600 & 0.889031 & -0.214505 & 2.027566 & 0.981902 & -0.382615 \\
\hline 1.666626 & 0.891541 & -0.219049 & 2.037592 & 0.984412 & -0.387158 \\
\hline 1.676653 & 0.894051 & -0.223592 & 2.047618 & 0.986922 & -0.391702 \\
\hline 1.686679 & 0.896561 & -0.228136 & 2.057644 & 0.989432 & -0.396245 \\
\hline 1.696705 & 0.899071 & -0.232679 & 2.067670 & 0.991942 & -0.400789 \\
\hline 1.706731 & 0.901581 & -0.237223 & 2.077696 & 0.994452 & -0.405332 \\
\hline 1.716757 & 0.904091 & -0.241766 & 2.087722 & 0.996962 & -0.409876 \\
\hline 1.726783 & 0.906601 & -0.246310 & 2.097748 & 0.999472 & -0.414419 \\
\hline 1.736809 & 0.909111 & -0.250853 & 2.107774 & 1.001982 & -0.418963 \\
\hline 1.746835 & 0.911621 & -0.255397 & 2.117800 & 1.004492 & -0.423506 \\
\hline
\end{tabular}

\section{D.5 Trajectory 5}

$\begin{array}{llllll}-0.880000 & 0.254000 & 0.935000 & -0.849982 & 0.265694 & 0.930898 \\ -0.869994 & 0.257898 & 0.933633 & -0.839977 & 0.269592 & 0.929531 \\ -0.859988 & 0.261796 & 0.932265 & -0.829971 & 0.273490 & 0.928164\end{array}$




\begin{tabular}{|c|c|c|c|c|c|}
\hline-0.819965 & 0.277389 & 0.926796 & -0.389714 & 0.445007 & \\
\hline-0.809959 & 0.281287 & 0.925429 & -0.379708 & 0.448905 & 0.866635 \\
\hline-0.799953 & 0.285185 & 24062 & -0.369702 & & \\
\hline-0.789947 & 0.289083 & 0.922694 & -0.359697 & 0.456701 & 0.863901 \\
\hline-0.779942 & 0.292981 & 0.921327 & -0.349691 & 0.460599 & 0.862533 \\
\hline-0.769936 & 0.296879 & 0.919960 & -0.339685 & 0.464497 & 0.861166 \\
\hline 930 & 0.300777 & 0.918592 & -0.329679 & 395 & 1799 \\
\hline & 304675 & & & & \\
\hline & .308573 & 15858 & -0.309667 & 0.476191 & 0.857064 \\
\hline & 2471 & & & & \\
\hline & 0.316369 & & & & \\
\hline-0.7 & 0.320268 & 1756 & -0.27 & 386 & 0.852962 \\
\hline 0.65 & 0.324166 & 0389 & 344 & 784 & 1595 \\
\hline-0.689889 & 0.328064 & 9021 & & 95682 & 228 \\
\hline-0.679883 & 0.331962 & 0.907654 & 632 & 580 & 860 \\
\hline 9877 & 0.335860 & 0.906287 & 327 & 478 & 493 \\
\hline 72 & 0.339758 & 0.90 & $\$ 21$ & 376 & 126 \\
\hline 66 & 0.343656 & 0.903552 & $\$ 15$ & 274 & 759 \\
\hline 860 & 0.347554 & 0.902185 & 609 & 172 & 391 \\
\hline 0.629854 & 0.351452 & 0.900818 & -0.199603 & 070 & 024 \\
\hline 848 & 0.355350 & 0.899450 & 9597 & 968 & 657 \\
\hline 42 & 0.359249 & 0.898083 & 592 & 866 & 289 \\
\hline & 33147 & 0.8 & & & 922 \\
\hline & 045 & & & & 555 \\
\hline & 70943 & & & & 188 \\
\hline & 374841 & 0.8 & & & 820 \\
\hline & 0.378739 & 0.89 & & 357 & 453 \\
\hline 07 & 0.382637 & 0.889879 & & 255 & 086 \\
\hline 9802 & 0.386535 & 0.888512 & & 53 & 718 \\
\hline 0.52 & 0.390433 & & & & \\
\hline 9790 & 0.39433 .1 & 777 & & & 984 \\
\hline & 0.398229 & & & & 616 \\
\hline-0.499778 & 402128 & .883043 & & & 249 \\
\hline-0.489772 & .406026 & .881676 & & 344 & 882 \\
\hline-0.479767 & 0.409924 & 0.88 & & & 515 \\
\hline & 0.413822 & 0.878941 & & & 147 \\
\hline 1755 & 0.417720 & & & & 780 \\
\hline-0.449749 & 0.421618 & & & & 413 \\
\hline-0.439743 & 0.425516 & & & & 0.816045 \\
\hline-0.429737 & 0.429414 & 0.873472 & & 0.597032 & 0.814678 \\
\hline-0.419732 & 0.433312 & 0.872104 & 0.010519 & 0.600330 & 0.813311 \\
\hline-0.409726 & 0.437210 & 0.870737 & 0.020525 & 0.604828 & 0.811944 \\
\hline$=0.399720$ & 0.441108 & 0.869370 & 0.030531 & 0.608726 & 0.810576 \\
\hline
\end{tabular}




\begin{tabular}{|c|c|c|c|c|c|}
\hline .040537 & 0.612625 & 0.809209 & 0.470788 & 0.780243 & \\
\hline .050543 & 0.616523 & 0.807842 & 0.480793 & 0.784141 & \\
\hline 060548 & 0.620421 & 0.806474 & 0.490799 & 0.788039 & \\
\hline 070554 & 0.624319 & 0.805107 & .500805 & 0.791937 & \\
\hline .080560 & 0.628217 & 0.803740 & 0.510811 & 0.795835 & \\
\hline 0.090566 & 0.632115 & 0.802372 & 0.520817 & & \\
\hline 0.100572 & 0.636013 & 0.801005 & 0.530823 & 0.803631 & \\
\hline 0.110578 & 0.639911 & 0.799638 & 0.540828 & 0.807529 & \\
\hline 0.120583 & 0.643809 & 0.798271 & 0.550834 & 0.811427 & \\
\hline 0.130589 & 0.647707 & 0.796903 & $0.560840^{\circ}$ & 0.815325 & \\
\hline 0.140595 & 0.65 & & & 9223 & \\
\hline 150601 & 0.65 & $0.7 s$ & & 3122 & \\
\hline 60607 & 0.659402 & 0.792801 & & 0.827 & \\
\hline 170613 & 0.663300 & 434 & 363 & 18 & \\
\hline 180618 & 0.667 & 0.790 & 369 & 0.834 & \\
\hline 624 & 0.67 & 0.78 & 75 & 0.85 & \\
\hline 0.200630 & 0.67 & 0.7 & & & \\
\hline 0.210636 & 0.67 & $0.7 \varepsilon$ & & 0.8 & \\
\hline 642 & 0.68 & & & & \\
\hline 648 & 0.6 & & & & \\
\hline 653 & 0.6 & & & & \\
\hline 0.250659 & 0.69 & 0.7 & & & \\
\hline 0.26 & 0.6 & & & & \\
\hline 671 & 281 & 761 & 22 & & \\
\hline 280677 & 179 & 394 & 328 & & \\
\hline .290683 & 077 & 027 & 933 & & \\
\hline 300688 & 0.71 & 659 & 939 & & \\
\hline 310694 & 0.71 & $0.77 i$ & 945 & & \\
\hline 320700 & 0.72 & 0.77 & 951 & 89 & \\
\hline 330706 & 0.725669 & 0.76 & 357 & 287 & \\
\hline 340712 & 0.729567 & 0.7681 & 363 & 85 & 0. \\
\hline 350718 & 0.733465 & 0.766823 & 968 & 83 & 0 . \\
\hline 0.360723 & 0.73 & 0.7 & & 381 & 0.7 \\
\hline 729 & 0.74 & 0.7 & & 380 & 0.7 \\
\hline & & 0.7 & & & \\
\hline 0.390741 & 0.74 & $0.7 \epsilon$ & & 76 & \\
\hline 400747 & 0.752956 & 0.75 & & 574 & \\
\hline 1.410753 & 0.756854 & 0.75 & & 0.924 & 0.6998 \\
\hline .420758 & 0.760752 & 0.75 & 009 & 0.928370 & 0.6984 \\
\hline .430764 & 0.764650 & 0.755884 & 0.861015 & 0.932268 & 0.6970 \\
\hline .440770 & 0.768548 & 0.754517 & 0.871021 & 0.936166 & \\
\hline 450776 & 0.772446 & 0.753150 & 0.881027 & 0.940064 & \\
\hline 460782 & 0.776344 & 0.751783 & 0.891033 & 0.943962 & 0.00 \\
\hline
\end{tabular}




\begin{tabular}{|c|c|c|c|c|c|}
\hline 0.901038 & 0.947861 & 0.691622 & 1.331289 & 1.115478 & 0.632828 \\
\hline 0.911044 & 0.951759 & 0.690254 & 1.341295 & 1.119377 & 0.631461 \\
\hline 0.921050 & 0.955657 & 0.688887 & 1.351301 & 1.123275 & 0.630093 \\
\hline 0.931056 & 0.959555 & 0.687520 & & & \\
\hline 0.941062 & 0.963453 & 0.686152 & 1.371313 & 1.131071 & \\
\hline 0.951068 & 0.967351 & 0.684785 & 1.381318 & 1.134969 & 0.625991 \\
\hline 0.961073 & 0.971249 & 0.683418 & 1.391324 & 1.138867 & 0.624624 \\
\hline 0.971079 & 0.975147 & 0.682051 & 1.401330 & 1.142765 & 0.623257 \\
\hline 0.981085 & 0.979045 & 0.680683 & 1.411336 & 1.146663 & 0.621890 \\
\hline 0.991091 & 0.982943 & 0.679316 & 1.421342 & 1.150561 & 0.620522 \\
\hline 1.001097 & 0.986841 & 0.677949 & 1.431348 & 1.154459 & 9155 \\
\hline 1.011103 & 0.990740 & 0.676581 & 1.441353 & 1.158358 & 0.617788 \\
\hline 1.021108 & 0.994638 & 0.675214 & 1.451359 & 1.162256 & 0.616420 \\
\hline 1.031114 & 0.998536 & 0.673847 & 1.461365 & 1.166154 & 0.615053 \\
\hline 1.041120 & 1.002434 & 0.672479 & 1.471371 & 1.170052 & 0.613686 \\
\hline 1.051126 & 1.006332 & 112 & 1.481377 & 1.173950 & 319 \\
\hline 1.061132 & 1.01 & 745 & 1.491383 & 1.17 & \\
\hline 1.071138 & 1.01 & 8378 & 1.501388 & 1.18 & 584 \\
\hline 1.081143 & 1.018026 & 010 & 1.511394 & 1.18 & 217 \\
\hline 1.091149 & 1.02 & 0.66 & 400 & 542 & \\
\hline 1.101155 & 1.02 & 0.6 & 406 & 440 & 482 \\
\hline 1.111161 & 1.02 & 0.66 & 412 & 338 & 115 \\
\hline 1.121167 & 1.03 & 0.6 & $\$ 18$ & 237 & 1747 \\
\hline 1.131173 & 1.037517 & 0.660174 & 1423 & 1.205135 & 0.601380 \\
\hline 1.141178 & 1.04 & & 429 & 1.20 & 0013 \\
\hline & & & 435 & 1.212931 & 8646 \\
\hline 1.16 & 1.04 & 0.6 & 1441 & 1.216829 & 0.597278 \\
\hline 1.171196 & 1.053109 & 0.654 & 1.601447 & 1.220727 & 0.595911 \\
\hline 1.181202 & 1.057007 & 0.653337 & 1.611453 & 1.224625 & 0.594544 \\
\hline 1.191208 & 1.060905 & 0.651970 & 1.621458 & 1.228523 & 0.593176 \\
\hline 1.201213 & 1.064803 & 0.650603 & 1.631464 & 1.232421 & 1809 \\
\hline 1.211219 & 1.068701 & 0.649235 & 1.641470 & 1.236319 & 0.590442 \\
\hline 1.221225 & 1.072599 & 0.647868 & 1.651476 & 1.240217 & 0.589075 \\
\hline 1.231231 & 1.076498 & 0.646501 & 1.661482 & 1.244116 & 0.587707 \\
\hline 1.241237 & 1.080396 & 0.645134 & 1.671488 & 1.248014 & 0.586340 \\
\hline 1.251243 & 1.084294 & 0.643766 & 1.681493 & 1.251912 & 0.584973 \\
\hline 1.261248 & 1.088192 & 0.642399 & 1.691499 & 1.255810 & 0.583605 \\
\hline 1.271254 & 1.092090 & 0.641032 & 1.701505 & 1.259708 & 0.582238 \\
\hline 1.281260 & 1.095988 & 0.639664 & 1.711511 & 1.263606 & 0.580871 \\
\hline 1.291266 & 1.099886 & 0.638297 & 1.721517 & 1.267504 & 0.579503 \\
\hline 1.301272 & 1.103784 & 0.636330 & 1.731523 & 1.271402 & 0.578136 \\
\hline 1.311278 & 1.107682 & 0.635563 & 1.741528 & 1.275300 & 0.576769 \\
\hline 1.321283 & .111580 & 0.634195 & 1.751534 & 1.279198 & 0.575402 \\
\hline
\end{tabular}




$\begin{array}{llllll}1.761540 & 1.283096 & 0.574034 & 1.811569 & 1.302587 & 0.567198 \\ 1.771546 & 1.286995 & 0.572667 & 1.821575 & 1.306485 & 0.565831 \\ 1.781552 & 1.290893 & 0.571300 & 1.831581 & 1.310383 & 0.564463 \\ 1.791558 & 1.294791 & 0.569932 & 1.841587 & 1.314281 & 0.563096 \\ 1.801563 & 1.298689 & 0.568565 & & & \end{array}$


ORNL/TM-13021

\section{INTERNAL DISTRIBUTION}

1. G. A. Armstrong

2. J. E. Baker

3. E. C. Bradley

4. B. L. Burks

5. J. B. Chesser

6. J. V. Draper

7. V. B. Graves

8. C. J. Hacker

9. D. C. Haley

10. W. R. Hamel

11. J. H. Hannah

12-14. J. N. Herndon

15. J. F. Jansen

16. S. M. Killough

17. R. L. Kress

18. C. T. Kring

19. M. W. Noakes
20. C. E. Oliver

21-25. F. G. Pin

26. A. H. Primm

27. B. S. Richardson

28. S. L. Schrock

29. S. Shekhar

30. K. U. Vandergriff

31. V.K. Varma

32. B. S. Weil

33. H. R. Yook

34-35. Laboratory Records

36. Laboratory Records ORNL - RC

37. RPSD Publications Office

38. ORNL Patent Section

39. Central Research Library

40. Document Reference Section

\section{EXTERNAL DISTRIBUTION}

41. Tobe Cookingham, 112 Upson Hall, Department of Mechanical and Aerospace Engineering, Cornell University, Ithaca, NY 14853

42-46. G. A. Fries, 1205 N. Belgrade Rd., Silver Spring, MD 20902

47. Sherman "Bud" Hayden, R\&M Technology Insertion, Hq Air Combat Command, Hq ACC/DRL, 204 Dodd Blvd., Suite 226, Langly AFB, VA 23665-2777

48. Dr. Pradeep Khosla, ARPA/SSTO, 3701 North Fairfax Dr., Arlington, VA 22203-1714

49. Major Michael Leahy, HQ AFMC/STC, 4375 Chidlaw Rd., Suite 6, WPAFB, OH 45433-5006

50. S. R. Martin, Jr., Acting Program Manager, Fusion and Nuclear Technology Branch, Energy Programs Division, Department of Energy, X-10 Site, Post Office Box 2008, Oak Ridge, TN 37831-6269

51. Dr. Matthew Miller, 194 Engineering and Theory Center Building, Department of Mechanical and Aerospace Engineering, Cornell University, Ithaca, NY 14853

52. Kristi Morgansen, Harvard Robotics Laboratory, Pierce Hall, 29 Oxford St., Harvard University, Cambridge, MA 02138 
53. Scott B. Petroski, Robotics and Automation Center of Excellence, San Antonio Air Logistics Center, SA/ALC/TIEST, Bldg. 183, 950 Quentin Roosevelt Road, Kelly Air Force Base, TX 78241-6416

54. Dean L. Taylor, 110 Upson Hall, Department of Mechanical and Aerospace Engineering, Cornell University, Ithaca, NY 14853

55. Dr. Kenneth E. Torrance, 242 Upson Hall, Department of Mechanical and Aerospace Engineering, Cornell University, Ithaca, NY 14853

56. Michael Toscano, OUSD (A\&T/S\&TS/LW), The Pentagon, Room 3B1060, Washington, DC 20310-3000

57. Faithlyn Tulloch, Escondido Village, Apt. 89C, Stanford, CA 94305

58. SMSG Tom Turner, AFSEO/SKZ, 207 W. D. Avenue, Suite 303, Eglin Air Force Base, FL 32542

59. Dr. Zellman Warhaft, 244 Upson Hall, Department of Mechanical and Aerospace Engineering, Cornell University, Ithaca, NY 14853

60. Office of Assistant Manager for Energy Research and Development, Oak Ridge Operations Office, Department of Energy, Post Office Box 2008, Oak Ridge, TN 37831-6269

61-62. Office of Scientific and Technical Information, Post Office Box 62, Oak Ridge, TN 37831 